\title{
EQUILIBRIUM STATES, PRESSURE AND ESCAPE FOR MULTIMODAL MAPS WITH HOLES
}

\author{
MARK F. DEMERS AND MIKE TODD
}

\begin{abstract}
For a class of non-uniformly hyperbolic interval maps, we study rates of escape with respect to conformal measures associated with a family of geometric potentials. We establish the existence of physically relevant conditionally invariant measures and equilibrium states and prove a relation between the rate of escape and pressure with respect to these potentials. As a consequence, we obtain a Bowen formula: we express the Hausdorff dimension of the set of points which never exit through the hole in terms of the relevant pressure function. Finally, we obtain an expression for the derivative of the escape rate in the zero-hole limit.
\end{abstract}

\section{INTRODUCTION}

For a class of dynamical systems with holes, we study the relation between the conditionally invariant measures, rates of escape and pressures with respect to a family of potentials. Given an interval map $f: I \circlearrowleft$ and a hole $H \subset I$, we define the exponential rate of escape with respect to a reference measure $m$ to be

$$
\mathfrak{e}(m, H)=-\lim _{n \rightarrow \infty} \frac{1}{n} \log m\left(\cap_{i=0}^{n} f^{-i}(I \backslash H)\right)
$$

when the limit exists. We say the open system satisfies a Variational Principle with respect to a potential $\phi$ if $-\mathfrak{e}(m, H)=P_{\mathcal{C}}(\phi)$ where $P_{\mathcal{C}}(\phi)$ denotes the pressure of $\phi$ taken over a class of relevant invariant measures $\mathcal{C}$,

$$
P_{\mathcal{C}}(\phi)=\sup _{\mu \in \mathcal{C}}\left\{h(\mu)+\int \phi d \mu\right\}
$$

We will focus on a class of multimodal Collet-Eckmann maps of the interval satisfying a slowrecurrence condition to the boundary of the hole. Such maps were studied in [BDM] using Lebesgue measure as a reference measure and $-\log |D f|$ as the relevant potential.

In this paper, we generalize this study to include the family of potentials $\left\{\varphi_{t}:=-t \log |D f|: t \in \mathbb{R}\right\}$. We will denote by $P_{\mathcal{M}_{f}}\left(\varphi_{t}\right)$ the pressure with respect to the potential $\varphi_{t}$ taken over all ergodic $f$-invariant probability measures, $\mathcal{M}_{f}$. These potentials are often referred to as geometric potentials since they capture the geometry and statistical growth properties of the system. For example, it was shown in $\left[\mathrm{L}\right.$ that a measure $\mu \in \mathcal{M}_{f}$ with positive entropy is an equilibrium state for $\varphi_{1}$ if and only if $\mu$ is absolutely continuous with respect to Lebesgue measure. Moreover, it was shown in [BK] (unimodal Collet-Eckmann case, restricted $t$ ), [BT] (multimodal case, restricted $t$ ) and [IT1] (multimodal case, general $t$ ) that there is an equilibrium state $\mu_{t}$ corresponding to $\varphi_{t}$. The relation between these measures, the pressure and the Lyapunov spectrum was shown in [IT2]. The

Date: April 6, 2016.

MD was partially supported by NSF grant DMS 1101572. MT was partially supported by NSF grants DMS 0606343 and DMS 0908093. Both authors would like to thank Boston University where part of this research was carried out. 
classical Bowen formula in the uniformly expanding case, see for example [Ra, LM], states that the Hausdorff dimension of the survivor set (the set of points which never escapes through the hole) is the value $t^{*} \geqslant 0$ such that $P_{\mathcal{M}_{f}}\left(\varphi_{t^{*}}\right)=0$.

In this paper we will fix a relevant reference measure and then look at how the mass given by this measure escapes through holes. When we consider the potentials $\varphi_{t}$ for $t \in \mathbb{R}$, our reference measure will be the corresponding $\left(\varphi_{t}-P_{\mathcal{M}_{f}}\left(\varphi_{t}\right)\right)$-conformal measure $m_{t}$. These were shown to exist in [IT3], and moreover for the equilibrium state $\mu_{t}$ for $\varphi_{t}$, we have $\mu_{t} \ll m_{t}$.

For this class of potentials and reference measures, we prove that the escape rate has a natural expression in terms of the pressure; we also prove the existence of further measures, one of which is an equilibrium state on the survivor set and one of which is the relevant 'geometric conditionally invariant measure' for the system. Such conditionally invariant measures, defined precisely in Section 2.4. describe the evolution of reasonable classes of initial distributions that have densities with respect to the conformal measures $m_{t}$. In addition, we are able to prove a Bowen formula for the Hausdorff dimension of the survivor set. Finally, we provide a formula for the derivative of the escape rate as our hole shrinks to a point (the zero-hole limit).

Similar results regarding the derivative of the escape rate were proved in [KL2, FP] using spectral theory. By contrast, in our setting no spectral picture is known for the transfer operators associated with our class of multimodal maps, so we construct Young towers instead. Unfortunately, a new Young tower must be constructed for each hole since return times can suffer unbounded changes due to arbitrarily small perturbations. Thus a principal aim of the present paper is to develop techniques which allow us to retain sufficient control of the towers we construct along a sequence of holes to prove results such as the Bowen formula and the derivative of the escape rate. We note that questions in thermodynamic formalism, such as multifractal spectra have been studied before in the context of multimodal maps, for example in [CT, IT2], this is the first proof of a Bowen formula for such a general class of maps. Moreover, our development of the theory of Young towers to tackle this problem gives a powerful abstract framework to deal with other non-uniformly hyperbolic dynamical systems.

The paper is organized as follows. In Section 2, we precisely define our class of maps, introduce our conditions on the types of holes we allow and recall definitions of the objects fundamental to the present work, including pressure, inducing schemes and Young towers. Section 3 contains a precise statement of our main results while Section 4 establishes that we have uniform control over our inducing schemes for a family of potentials. In Section 5 we recall some facts from [BDM] regarding abstract towers with holes and in Section 6 we show how to apply those results to our present setting. Finally, we prove our Variational Principle in Section 7 and a Bowen formula for the Hausdorff dimension of the survivor set in Section 8. Section 9 contains the proof of the derivative of the escape rate in the zero-hole limit.

\section{Setup}

2.1. Multimodal interval maps with some exponential growth. Collet-Eckmann maps are interval maps $f: I \circlearrowleft$ with critical points such that the derivatives $D f^{n}$ at the critical values increase exponentially. We will follow the approach of BDM, DHL which allows for multimodal maps with singularities (in this case our singularity set will be the boundary of the hole).

We say a critical point $c$ has critical order $\ell_{c}>0$ if there exists a neighborhood $U_{c}$ of $c$ and a diffeomorphism $g_{c}: U_{c} \rightarrow g_{c}\left(U_{c}\right)$ such that $g_{c}(c)=0$ and $f(x)=f(c) \pm\left|g_{c}(x)\right|^{\ell_{c}}$ for all $x \in U_{c}$. A critical point $c$ is non-flat if $\ell_{c}<\infty$. 
In this paper, we assume the map $f: I \rightarrow I$ is topologically mixing and $\mathcal{C}^{2}$ with a critical set Crit ${ }_{c}$ consisting of finitely many critical points $c$ with critical order $2 \leqslant \ell_{c}<\infty$. Note that in particular, topological mixing means our maps are non-renormalizable: we make this assumption to avoid technicalities regarding uniqueness of equilibrium states. Let $B_{\delta}\left(\mathrm{Crit}_{c}\right)=\cup_{c \in \mathrm{Crit}_{c}} B_{\delta}(c)$ denote the $\delta$-neighborhood of $\mathrm{Crit}_{c}$. We assume $f$ satisfies the following conditions for all sufficiently small $\delta>0$ :

(C1) Expansion outside $B_{\delta}\left(\right.$ Crit $\left._{c}\right)$ : There exist $\gamma>0$ and $\kappa>0$ such that for every $x$ and $n \geq 1$ such that $x_{0}=x, \ldots, x_{n-1}=f^{n-1}(x) \notin B_{\delta}\left(\right.$ Crit $\left._{c}\right)$, we have

$$
\left|D f^{n}(x)\right| \geq \kappa \delta^{\ell_{\max }-1} e^{\gamma n},
$$

where $\ell_{\max }=\max \left\{\ell_{c}: c \in \mathrm{Crit}_{c}\right\}$. Moreover, if $x_{0} \in f\left(B_{\delta}\left(\mathrm{Crit}_{c}\right)\right)$ or $x_{n} \in B_{\delta}\left(\mathrm{Crit}_{c}\right)$, then we have

$$
\left|D f^{n}(x)\right| \geq \kappa e^{\gamma n}
$$

(C2) Slow recurrence and derivative growth along critical orbit: There exists $\Lambda>0$ such that for all $c \in$ Crit $_{c}$ there is $\vartheta_{c} \in\left(0, \Lambda /\left(5 \ell_{c}\right)\right)$ such that

$$
\left|D f^{k}(f(c))\right| \geq e^{\Lambda k} \text { and } \operatorname{dist}\left(f^{k}(c), \operatorname{Crit}_{c}\right)>\delta e^{-\vartheta_{c} k} \quad \text { for all } k \geq 1 .
$$

A consequence of $(\mathrm{C} 1)$ and $(\mathrm{C} 2)$ together is that all periodic orbits must be repelling. The first half of condition (C2) is the actual Collet-Eckmann condition, and the second half is a slow recurrence condition 1$]$

We assume without loss of generality that $\vartheta_{c}$ is small relative to $\gamma$ and $\Lambda$.

2.2. Introduction of Holes. A hole $H$ in $I$ is a finite union of open intervals $H_{j}, j=1, \ldots, L$. Let $\stackrel{\circ}{I}=I \backslash H$ and set $\stackrel{\circ}{I}^{n}=\bigcap_{i=0}^{n} f^{-i} \stackrel{\circ}{I}, n \in \mathbb{N} \cup\{\infty\}$. We refer to the set $\stackrel{\circ}{I}^{\infty}$ as the survivor set for the open system, i.e., $\stackrel{\circ}{I}^{\infty}$ represents the set of points that do not escape in forward time. Define $f^{n}=f^{n} \mid I^{n}, n \geqslant 1$, to be the maps on the noninvariant domains $\stackrel{\circ}{I}^{n}$.

Two objects fundamental to the study of open systems are the escape rate $\mathfrak{e}(m, H)$ defined by (1) and conditionally invariant measures, whose definition we recall below. In what follows, in order to simplify notation when the hole is clear by context, we sometimes suppress that variable and denote the escape rate by $\mathfrak{e}(m)$.

A conditionally invariant measure for the open system $(I, f, H)$ is a probability measure $\mu$ for which there exists a constant $0 \leqslant \lambda<1$ such that $\stackrel{\circ}{*}_{*} \mu(A):=\mu\left(f^{-1} A \cap \stackrel{\circ}{1}^{1}\right)=\lambda \mu(A)$ for any Borel set $A \subset I$. This relation immediately implies $\lambda=\mu\left(\stackrel{\circ}{I}^{1}\right)$ and $f_{*}^{n} \mu(A)=\lambda^{n} \mu(A)$ so that $\mathfrak{e}(\mu)=-\log \lambda$ by (1).

In order to invoke the tower construction of [BDM], we place several conditions on the placement of the holes in the interval $I$.

(H1) Let $\vartheta_{c}, \delta>0$ be as in (C2). For all $c \in \mathrm{Crit}_{c}$ and $k \geq 0$,

$$
\operatorname{dist}\left(f^{k}(c), \partial H\right)>\delta e^{-\vartheta_{c} k} \text {. }
$$

(H1) and (C2) imply that we can treat $\partial H$ the same as Crit $_{c}$ in terms of the slow approach of critical orbits. Our second condition on $H$ is that the positions of its connected components are generic with

\footnotetext{
${ }^{1}[\mathrm{DHL}]$ and $[\mathrm{BDM}]$ include a third condition as part of their formal assumptions: (C3) $\exists c^{*} \in$ Crit $_{c}$ whose preimages are dense in $I$ and no other critical point is among these preimages. In our setting, it follows from (C1)-(C2) and our assumption of topological mixing that all $c \in \mathrm{Crit}_{c}$ satisfy this condition.
} 
respect to one another. This condition also doubles as a transitivity condition on the constructed tower which ensures our conditionally invariant densities will be bounded away from zero. In order to formulate this condition, we need the following fact about $\mathcal{C}^{2}$ nonflat nonrenormalizable maps satisfying (C1)-(C2) (see [BDM, Sect. 2.4.2] or [DHL, Lemma 1]).

There exist $c^{*} \in$ Crit and $\delta_{*}>0$ such that for all $\delta \leqslant \delta_{*}$, there exists $n=n(\delta)$

such that for all intervals $\omega \subseteq I$ with $|\omega| \geq \frac{\delta}{3}$,

(i) $f^{n} \omega \supseteq I$, and

(ii) there is a subinterval $\omega^{\prime} \subset \omega$ such that $f^{n^{\prime}}$ maps $\omega^{\prime}$

diffeomorphically onto $\left(c^{*}-3 \delta, c^{*}+3 \delta\right)$ for some $0<n^{\prime} \leq n$.

Using this fact, we formulate a condition on the placement of the components of the hole. This condition is generic in the sense that it is satisfied by a full-measure set of parameters governing the placement. Within each component $H_{j}$, we place an artificial critical point $b_{j}$, so

$$
\text { Crit }_{\text {hole }}:=\left\{b_{1}, \ldots, b_{L}\right\} \text {. }
$$

The points $b_{j}$ are chosen so that Crit $_{\text {hole }} \cap$ Crit $_{c}=\emptyset$. Choose $\delta$ so small that all points in Crit $_{c} \cup$ Crit $_{\text {hole }}$ are at least $\delta$ apart and let $n(\delta)$ be the corresponding integer from (2). We assume the following.

(H2)

(a) $\left(\cup_{n \geqslant 0} f^{n} b_{j}\right) \cap c \in$ Crit $_{c}=\emptyset$ for all $1 \leq j \leq L$.

(b) Let $f^{-1}\left(f b_{j}\right)=\cup_{i=1}^{K_{j}} g_{j, i}$. For all $j, k \in\{1, \ldots, L\}$, there exists $i \in\left\{1, \ldots, K_{j}\right\}$ such that $f^{\ell} b_{k} \neq g_{j, i}$ for $1 \leq \ell \leq n(\delta)$.

(c) For each $j=1, \ldots, L$, there is $r=r(j)$ such that for all $x \in B_{\delta}\left(b_{j}\right), f^{i}(x) \notin$ $B_{\delta}\left(\mathrm{Crit}_{c} \cup\right.$ Crit $\left._{\text {hole }}\right)$ for $i=1, \ldots, r(j)-1$, and $\left|D f^{r}(x)\right| \geq \max \left\{\kappa e^{\gamma r}, 4\right\}$.

For generically placed holes, Condition (C1) implies $\left|D f^{r}(x)\right| \geq \kappa e^{\gamma r}$ whenever $x \notin B_{\delta}\left(\mathrm{Crit}_{c}\right)$ and $f^{r}(x) \in B_{\delta}\left(\right.$ Crit $\left._{c}\right)$, so by taking $\delta$ small, and using assumption (H2)(a), we can always satisfy $(\mathrm{H} 2)(\mathrm{c})$. The specific form of $(\mathrm{H} 2)(\mathrm{c})$ is to allow the $b_{j}$ to be periodic points, which is the one point of difference with $[\mathrm{BDM}]$ in this condition.

2.3. Pressure and conformal measures. Suppose that $f: X \rightarrow X$ is a dynamical system on a topological space $X$ and $\phi: X \rightarrow[-\infty, \infty]$ is a potential, both of these maps preserving the Borel structure. Then we define the pressure of $\phi$ to be

$$
P_{\mathcal{M}_{f}}(\phi):=\sup _{\mu \in \mathcal{M}_{f}}\left\{h(\mu)+\int \phi d \mu:-\int \phi d \mu<\infty\right\}
$$

where

$$
\mathcal{M}_{f}:=\left\{\mu \text { Borel, ergodic, } \mu \circ f^{-1}=\mu, \mu(X)=1\right\},
$$

and $h(\mu)$ denotes the (metric) entropy of $\mu$. If a measure $\mu \in \mathcal{M}_{f}$ satisfies $h(\mu)+\int \phi d \mu=P_{\mathcal{M}_{f}}(\phi)$, then we call $\mu$ an equilibrium state for $(X, f, \phi)$. These measures are often associated with another natural type of (possibly non-invariant) measure: a Borel measure $m$ on $X$ is called $\phi$-conformal if the Jacobian of $m$ is $e^{\phi}$, i.e., $\frac{d m}{d(m \circ f)}=e^{\phi}$.

In this paper we will be particularly interested in the set of interval maps defined above and in the potential $\varphi:=-\log |D f|$ as well as the family

$$
\left\{\varphi_{t}:=-t \log |D f|: t \in \mathbb{R}\right\} .
$$

We will sometimes denote $p_{t}:=P_{\mathcal{M}_{f}}\left(\varphi_{t}\right)$ to be the pressure with respect to the potential $\varphi_{t}$. 
For the potential $\varphi=\varphi_{1}$, the natural reference measure is $m=$ Lebesgue, with respect to which the equilibrium state $\mu$ for $(I, f, \varphi)$ is absolutely continuous. Notice that Lebesgue is $\varphi$-conformal, and indeed since $p_{1}=0$, it is trivially $\left(\varphi-p_{1}\right)$-conformal. This case was studied in [BDM]. When we consider the potentials $\varphi_{t}$ for $t \in \mathbb{R}$, our reference measure will be the corresponding $\left(\varphi_{t}-p_{t}\right)$ conformal measure $m_{t}$. These were shown to exist in [IT3], and moreover the equilibrium state $\mu_{t}$ for $\varphi_{t}$ satisfies $\mu_{t} \ll m_{t}$. For convenience, for $t \in \mathbb{R}$ we denote

$$
\phi_{t}:=\varphi_{t}-p_{t} .
$$

Given a potential $\phi$ on $I$, when we introduce a hole $H$ into the interval, we define the corresponding punctured potential by $\phi^{H}(x)=\phi(x)$ on $I \backslash H$ and $\phi^{H}(x)=-\infty$ on $H$; the corresponding set of measures is

$$
\mathcal{M}_{f}^{H}:=\left\{\mu \in \mathcal{M}_{f}: \mu(H)=0\right\} .
$$

Observe that by invariance, these measures must be supported on $\stackrel{\circ}{I}^{\infty}$.

In order for the pressure with respect to our punctured potential to be well-defined, we will have to restrict our class of invariant measures further. Define

$$
\mathcal{G}_{f}^{H}=\left\{\mu \in \mathcal{M}_{f}^{H}: \exists C, \beta>0 \text { such that for all } \varepsilon>0, \mu\left(B_{\varepsilon}(\partial H)\right) \leqslant C \varepsilon^{\beta}\right\} .
$$

The corresponding pressure we shall work with is,

$$
P_{\mathcal{G}_{f}^{H}}(\phi)=\sup _{\mu \in \mathcal{G}_{f}^{H}}\left\{h(\mu)+\int \phi d \mu:-\int \phi d \mu<\infty\right\} .
$$

The class of measures $\mathcal{G}_{f}^{H}$ are those invariant measures which do not concentrate too much mass on the boundary of the hole. As we will see in Section 7, the relevant measures here, for example the equilibrium state for $\phi_{t}^{H}$, lie in $\mathcal{G}_{f}^{H}$, so focusing on these measures is not a significant restriction.

2.4. Transfer Operators. We will study the statistical properties of our open systems via transfer operators both for $(I, f, H)$ and for the associated Young tower, defined in the next section.

Given a potential $\phi$ on $I$ and a suitable test function $\psi$, the associated transfer operator $\mathcal{L}_{\phi}$ acts on $\psi$ by $\mathcal{L}_{\phi} \psi(x)=\sum_{y \in f^{-1} x} \psi(y) e^{\phi(y)}$. When we work with the corresponding punctured potential $\phi^{H}$, we define the transfer operator in terms of the restricted map $f$ :

$$
\mathcal{L}_{\phi^{H}} \psi(x)=\sum_{y \in f^{-1} x} \psi(y) e^{\phi^{H}(y)}=\sum_{y \in f^{-1} x} \psi(y) e^{\phi(y)} 1_{\check{I}^{1}}(y),
$$

where $1_{A}$ denotes the indicator function of the set $A$.

The importance of the transfer operator stems from the fact that if $m$ is $\phi$-conformal and $g$ is a function such that $\mathcal{L}_{\phi^{H}} g=\lambda g$ for some $\lambda>0$, then $g m$ defines a conditionally invariant measure for $(I, f, H)$ with eigenvalue $\lambda$ :

$$
g m\left(\stackrel{\circ}{f}^{-1} A\right)=\int_{f^{-1} A \cap I^{1}} g d m=\int_{A \cap \stackrel{I}{I}} \mathcal{L}_{\phi^{H}} g d m=\lambda \int_{A \cap \stackrel{I}{I}} g d m=\lambda g m(A),
$$

where in the last step we have used the fact that $g$ is necessarily zero on $H$ due to the relation $\mathcal{L}_{\phi^{H}} g=\lambda g$.

Since for given $(I, f, H)$, many conditionally invariant measures exist for any eigenvalue between 0 and 1 under very mild conditions [DY], it is imperative to find a conditionally invariant measure with physical properties, such as that $\mu$ is the limit of $f_{*}^{n} m /\left|f_{*}^{n} m\right|$ for $m$ in a reasonable class of initial distributions. 
When our reference measure is the conformal measure $m_{t}$, we will take as our class of initial distributions those measures $\eta$ having Hölder continuous densities with respect to $m_{t}$. If a conditionally invariant measure $\mu_{t}^{H}$ can be realized as the limit of $\AA_{*}^{n} \eta /\left|\dot{f}_{*}^{n} \eta\right|$ for all such measures $\eta$, then we will call $\mu_{t}^{H}$ a geometric conditionally invariant measure. When $t=1$, such measures have been termed 'physical conditionally invariant measures' (see [BDM, DY]); we prefer the term 'geometric' in this context, since although we will prove that such $\mu_{t}^{H}$ have densities with respect to $m_{t}$, they are singular with respect to Lebesgue measure when $t \neq 1$.

2.5. Induced maps and Young towers. Given a set Crit ${ }_{\text {hole }}$ satisfying assumption (H2), in [BDM], inducing schemes $(X, F, \tau, H)$ are constructed respecting small holes $H$ satisfying (H1) and (H2). For an interval $X \subset I$, the triple $(X, F, \tau)$ is an inducing scheme if there is a countable collection of subintervals $\left\{X_{i}\right\}_{i} \subset X$ and a function $\tau: \cup_{i} X_{i} \rightarrow \mathbb{N}$ such that for each $i,\left.\tau\right|_{X_{i}}$ is constant and the map $F=\left.f^{\tau}\right|_{X_{i}}$ is a diffeomorphism of $X_{i}$ onto $X$. We define $\tau_{i}:=\left.\tau\right|_{X_{i}}$.

By 'respecting the hole' $H$, we mean that for each domain $X_{i}$ in the inducing scheme, either $f^{n}\left(X_{i}\right) \subset H$ or $f^{n}\left(X_{i}\right) \cap H=\emptyset$ for $0 \leqslant n \leqslant \tau\left(X_{i}\right)$. To accomplish this, $\partial H$ is considered as a discontinuity set for $f$ and cuts are introduced during the construction of the inducing scheme. During the construction, no escape is allowed and the holes are inserted afterwards into the tower $\Delta$ defined below.

Given an inducing scheme respecting a hole $H,(X, F, \tau, H)$, we define the corresponding Young tower as follows. Let

$$
\Delta=\{(x, n) \in X \times \mathbb{N} \mid n<\tau(x)\} .
$$

$\Delta$ is viewed schematically as a tower with $\Delta_{\ell}=\left.\Delta\right|_{n=\ell}$ as the $\ell$ th level of the tower. The tower map, $f_{\Delta}$, is defined by $f_{\Delta}(x, \ell)=(x, \ell+1)$ if $\ell+1<\tau(x)$ and $f_{\Delta}(x, \tau(x)-1)=\left(f^{\tau}(x), 0\right)=(F(x), 0)$ at return times. There is a canonical projection $\pi: \Delta \rightarrow I$ satisfying $\pi \circ f_{\Delta}=f \circ \pi . \Delta_{0}$ is identified with $X$ so that $\left.\pi\right|_{\Delta_{0}}=I d$. The partition $\left\{X_{i}\right\}$ induces a countable Markov partition $\left\{\Delta_{\ell, j}\right\}$ on $\Delta$ via the identification $\Delta_{\ell, j}=f_{\Delta}^{\ell}\left(X_{j}\right)$, for $\ell<\tau\left(X_{j}\right)$. The towers constructed in [BDM] are mixing, i.e., g.c.d. $\{\tau\}=1$, and the partition $\left\{X_{i}\right\}$ is generating.

If $f^{n}(x) \in H$, then we place a hole $\tilde{H}$ in $\Delta_{n}$ and the elements above $\tilde{H}$ in the tower are deleted: i.e., the set that maps into $\tilde{H}$ does not return to the base. The fact that the inducing scheme respects $H$ implies that $\tilde{H}:=\pi^{-1} H$ is the union of countably many partition elements $\Delta_{\ell, j}$. We

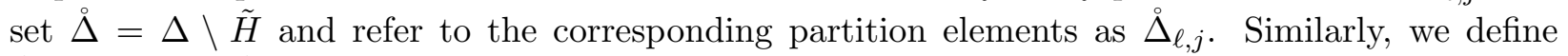
$\stackrel{\circ}{\Delta}^{n}=\cap_{i=0}^{n} f_{\Delta}^{-i} \stackrel{\circ}{\Delta}, n \in \mathbb{N} \cup\{\infty\}$.

2.6. Lifting to the Young tower. Since our main results are all proved using a Young tower $\Delta$ coming from an inducing scheme $(X, F, \tau, H)$, we will need to ensure that the tower we choose 'sees all the relevant statistical properties' of our system. In particular, we will show that the Hausdorff dimension of the set of points which do not return to $X$ with $F$ :

$$
\mathcal{N} \mathcal{R}_{\Delta}:=\{x \in X: \tau(x)=\infty\}
$$

is a set with Hausdorff dimension 'sufficiently bounded away from 1'; indeed Theorem 3.1 below bounds this by some $D<1$. This means that the Young tower contains all information in $I$ of sufficiently high Hausdorff dimension: we call points $x \in \pi(\Delta)$ liftable and denote this set by $\mathcal{R}_{\Delta}$. By topological transitivity there exists $N \in \mathbb{N}$ such that $f^{N}(X)=I$, and since Hausdorff dimension is preserved by bi-Lipschitz mappings, Theorem 3.1 says $\operatorname{dim}_{H}\left(f^{N}\left(\mathcal{N} \mathcal{R}_{\Delta}\right)\right) \leqslant D$, where $\operatorname{dim}_{H}(\cdot)$ denotes the Hausdorff dimension of a set. Therefore, the set of points in $I$ which are not liftable must have Hausdorff dimension $\leqslant D$. This means that if we were interested in a set $A \subset I$ that 
has Hausdorff dimension greater than $D$ then it must be 'seen by the tower':

$$
\operatorname{dim}_{H}\left(A \cap \mathcal{R}_{\Delta}\right)=\operatorname{dim}_{H}(A) .
$$

We will use this information on dimension in conjunction with measures. Setting $\tau(x)=\infty$ for all points for which $\tau(x)$ is not originally defined, we say that a measure $\mu$ on $I$ lifts to the inducing scheme (correspondingly lifts to the tower $\Delta$ ) if $\mu(X)>0$ and $\mu(\tau)<\infty$. If $\mu$ is an $f$-invariant measure which lifts, then according to [Z, Theorem 1.1], there is an induced measure $\nu \ll \mu$ supported on $X$ which is $F$-invariant and such that for any $A \subset I$,

$$
\mu(A)=\frac{1}{\int_{X} \tau d \nu} \sum_{i} \sum_{k=0}^{\tau_{i}-1} \nu\left(f^{-k}(A) \cap X_{i}\right)=\frac{1}{\int_{X} \tau d \nu} \sum_{k \geqslant 0} \nu\left(\{\tau>k\} \cap f^{-k}(A)\right) .
$$

Conversely, given an $F$-invariant measure $\nu$ such that $\int \tau d \nu<\infty$, there exists an $f$-invariant measure $\mu$ defined by (4) and we say that $\nu$ projects to $\mu$.

We can also consider the intermediate measures on the Young tower here: given an $F$-invariant measure $\nu$ with $\int \tau d \nu<\infty$, we can define a measure $\mu_{\Delta}^{\prime}$ on $\Delta$ by putting $\left.\mu_{\Delta}^{\prime}\right|_{\Delta_{0}}$ to simply be a copy of $\nu$ and then for any $A \subset \Delta_{\ell, i}$ where $0 \leqslant \ell \leqslant \tau_{i}-1$, since $f_{\Delta}^{-\ell}(A) \subset \Delta_{0}$, we can set $\mu_{\Delta}^{\prime}(A)$ to be $\mu_{\Delta}^{\prime}\left(f_{\Delta}^{-\ell}(A)\right)$. Then we obtain $\mu_{\Delta}:=\frac{1}{\int \tau d \nu} \mu_{\Delta}^{\prime}$. Observe that $\mu$ as in (4) is now the push-forward of $\mu_{\Delta}$ by $\pi$.

We set the pressure of $\phi$ on $\Delta$ to be

$$
P_{\Delta}(\phi):=\sup _{\mu \in \mathcal{M}_{f}}\left\{h(\mu)+\int \phi d \mu: \mu \text { lifts to } \Delta \text { and } \mu(-\phi)<\infty\right\} .
$$

Clearly $P_{\mathcal{M}_{f}}(\phi) \geqslant P_{\Delta}(\phi)$ for any $\phi$. Letting $\mathcal{M}_{F}$ be the set of $F$-invariant probably measures, we can define the pressure $P_{\mathcal{M}_{F}}$ as usual. To see the relation between these different pressures, for a potential $\phi: I \rightarrow[-\infty, \infty]$, define

$$
S_{n} \phi=\sum_{k=0}^{n-1} \phi \circ f^{k}
$$

Then we obtain the induced potential $\Phi(x)$ as $S_{\tau_{i}} \phi(x)$ for $x \in X_{i}$. Abramov's formula says that if $\mu$ lifts to $\nu$ then

$$
h(\mu)=\frac{h(\nu)}{\int \tau d \nu} \quad \text { and } \quad \int \phi d \mu=\frac{\int \Phi d \nu}{\int \tau d \nu} .
$$

This also holds for measures on $\Delta$, since any invariant probability measure $\mu_{\Delta}$ on $\Delta$ must lift to the corresponding inducing scheme; indeed the lifted measure is simply $\nu=\frac{\left.\mu_{\Delta}\right|_{\Delta_{0}}}{\mu_{\Delta}\left(\Delta_{0}\right)}$, the conditional measure on $\Delta_{0}$. The above relations then hold due to the extra information provided by Kac's Lemma that $\int_{\Delta_{0}} \tau d \nu=\frac{1}{\mu_{\Delta}\left(\Delta_{0}\right)}$.

We inductively define $\tau^{n}(x)=\tau^{n-1}(x)+\tau\left(f^{\tau^{n-1}(x)}(x)\right)$ to be the $n$th return of $x$ to $X$ under the inducing scheme, and let $\left\{X_{k, i}\right\}_{i}$ be the set of $k$-cylinders (cylinders of length $k$ ) for $(X, F)$ : that is, for each $i, \tau^{k}$ is constant on $X_{k, i}$ and $F^{k}: X_{k, i} \rightarrow X$ is a diffeomorphism. Moreover, for a potential $\Phi: \cup_{i} X_{i} \rightarrow \mathbb{R}$, define the $n$-th variation of $\Phi$ as

$$
V_{n}(\Phi):=\sup \{|\Phi(x)-\Phi(y)|: x, y \text { are contained in the same } n \text {-cylinder }\} .
$$

Then $\Phi$ is said to be locally Hölder if there exists $\eta>0$ such that $V_{n}(\Phi)=O\left(e^{-\eta n}\right)$ for all $n \in \mathbb{N}$. This condition on potentials is required to define the following kind of measure. We say that $\nu$ is 
a Gibbs measure for the potential $\Phi$ if $\nu(-\Phi)<\infty$ and there exist constants $C>0, P \in \mathbb{R}$, such that for each $n$-cylinder $X_{n, i}$ with respect to $F$, we have

$$
C^{-1} e^{S_{n} \Phi\left(y_{i}\right)-n P} \leqslant \nu\left(X_{n, i}\right) \leqslant C e^{S_{n} \Phi\left(y_{i}\right)-n P}
$$

for any $y_{i} \in X_{n, i}$, where $S_{n} \Phi=\sum_{k=0}^{n-1} \Phi \circ F^{k}=\sum_{k=0}^{\tau^{n}-1} \phi \circ f^{k}$. Note that the induced potentials considered in this paper will be locally Hölder and have equilibrium states which are Gibbs measures with the constant $P$ above equal to the pressure.

Remark 2.1. By $[\mathrm{Pr}]$, given $f$ as above, any $\mu \in \mathcal{M}_{f}$ has $\int \log |D f| d \mu \in\left[0,|D f|_{\infty}\right]$. Therefore our potentials $\varphi_{t}$ all have $\int \varphi_{t} d \mu$ finite. Thus we can drop the condition $-\int \varphi_{t} d \mu<\infty$ in the definition of pressure $P_{\mathcal{M}_{f}}\left(\varphi_{t}\right)$. Moreover, since as described above any $f_{\Delta}$-invariant measure $\mu_{\Delta}$ with $\mu_{\Delta}(\Delta)=1$ projects to a measure $\mu \in \mathcal{M}_{f}$, the same conclusion can be drawn for measures on $\Delta$ (see, in particular, Lemma 7.1). Notice, however, that for any of our inducing schemes $(X, F, \tau)$, there are measures $\mu_{F} \in \mathcal{M}_{F}$ that have $\int \log |D F| d \mu_{F}=\infty$. While we need to keep these in mind when computing $P_{\mathcal{M}_{F}}$, these measures will not to be relevant here.

\section{MAin Results}

We fix a set Crithole as described in Section 2.2 and $\delta_{0}>0$ sufficiently small such that (H2) and (2) are satisfied for $\delta=\delta_{0}$. This in turn fixes all $\delta$ appearing in $(\mathrm{C} 1)-(\mathrm{C} 2)$ to have value $\delta_{0}$.

For this choice of $\mathrm{Crit}_{\text {hole }}$, for $h>0$, define $\mathcal{H}(h)$ to be the family of holes $H \subset I$ such that

(1) $b_{j} \in H_{j}$ and $m_{1}\left(H_{j}\right) \leqslant h$ for each $j=1, \ldots, L$;

(2) $H$ satisfies (H1),

where $m_{1}$ denotes Lebesgue measure (the $\varphi_{1}$-conformal measure).

Theorem 3.1. Let $f$ satisfy (C1)-(C2) and fix the set Crithole as above. There exist constants $0<t_{0}<1<t_{1}$ and $h>0$ such that if $t \in\left[t_{0}, t_{1}\right]$ and $H \in \mathcal{H}(h)$, then $f$ admits an inducing scheme $(X, F, \tau, H)$ that respects the hole and

(a) there exists $D<1$ such that $\operatorname{dim}_{H}\left(\mathcal{N R}_{\Delta}\right) \leqslant D$;

(b) $\operatorname{dim}_{H}\left(\stackrel{\circ}{\Delta}^{\infty}\right)=\operatorname{dim}_{H}\left(\stackrel{\circ}{I}^{\infty}\right)$;

(c) $t \in\left[t_{0}, t_{1}\right]$ implies that $m_{t}(\tau<\infty)=m_{t}(X)$;

(d) if $t \in\left[t_{0}, t_{1}\right]$ then any measure $\mu$ on $I^{\infty}$ which doesn't lift to $\left(\Delta, f_{\Delta}\right)$ must have $h(\mu)+$ $\int \varphi_{t}^{H} d \mu<P_{\Delta}\left(\varphi_{t}^{H}\right)$.

We remark that (a), which is the solution of a problem of the liftability of measures, was used in [PS, Theorem 7.6] applying results of [Se]. As can be seen from the proof, the constant $D$ there is of the form $\log K /\left(\alpha_{1}+\log K\right)$ where $\alpha_{1}$ is the exponential return rate in the inducing scheme and $K$ depends on the complexity of the map, including the placement of the hole.

Proposition 3.2. Let $f, t_{0}<t_{1}, H$ and $\Delta$ be as in Theorem 3.1. Then there exist constants $C_{0}, \alpha>0$, depending only on $t_{0}, t_{1}$ and $h$ such that for each $t \in\left[t_{0}, t_{1}\right]$ we have $m_{t}(\tau>n) \leqslant C_{0} e^{-\alpha n}$, for all $n \geqslant 0$.

We denote by $\mathcal{C}^{p}(I)$ the space of Hölder continuous functions on $I$ with exponent $p$. In what follows, we assume that $p \geqslant \beta / \log \xi$, where $\xi>1$ is defined in (A1) in Section 4.2 and $0<\beta<\alpha$ from Section 5 defines the metric on $\Delta \cdot t^{2}$ For relevant $t$, let $g_{t}^{0}$ denote the density of the equilibrium

\footnotetext{
${ }^{2}$ In fact, $\beta$ can be chosen as small as one likes, allowing $p$ to be arbitrarily small. The price to pay is that then $h$ must be small according to (P4) of Section 5
} 
measure $\mu_{t}$ for $f$ with respect to $m_{t}$. Note that $g_{t}^{0} \notin \mathcal{C}^{p}(I)$ due to spikes corresponding to the critical orbits.

Theorem 3.3. Let $f, t_{0}<t_{1}$ and $H$ be as in Theorem 3.1 and recall that $\phi_{t}=t \varphi-p_{t}$. Then for each $t \in\left[t_{0}, t_{1}\right], \mathcal{L}_{\phi_{t}^{H}}$ has a unique simple eigenvalue $\lambda_{t}^{H} \leqslant 1$ of maximum modulus whose corresponding eigenvector $g_{t}^{H}$ defines an absolutely continuous conditionally invariant measure $\mu_{t}^{H}=g_{t}^{H} m_{t}$.

In addition, there exist constants $C_{*}>0, \sigma<1$ such that for any $\psi \in \mathcal{C}^{p}(I)$ with $\psi>0$,

$$
\lim _{n \rightarrow \infty}\left|\frac{\mathcal{L}_{\phi_{t}^{H}}^{n} \psi}{\left|\mathcal{L}_{\phi_{t}^{H}}^{n} \psi\right|_{L^{1}\left(m_{t}\right)}}-g_{t}^{H}\right|_{L^{1}\left(m_{t}\right)} \leqslant C_{*} \sigma^{n}|\psi|_{\mathcal{C}^{p}(I)} \quad \text { for all } n \geq 0 .
$$

The same limit holds for the sequence $\frac{\mathcal{L}_{\phi_{t}^{H}}^{n}\left(\psi g_{t}^{0}\right)}{\left.\mathcal{L}_{\phi_{t}^{H}}^{n}\left(\psi g_{t}^{0}\right)\right|_{L^{1}\left(m_{t}\right)}}$ and all positive $\psi \in \mathcal{C}^{p}(I)$. In particular $\mathfrak{e}\left(\psi m_{t}\right)=\mathfrak{e}\left(\psi \mu_{t}\right)=-\log \lambda_{t}^{H}$ for all $\psi \in \mathcal{C}^{p}(I), \psi>0$.

In fact, the limit (5) holds for any $\psi$ which projects down from the relevant function space on the tower. Since the result in this generality is technical to state, we refer the reader to Proposition 6.3 and Remark 6.4 for more precise statements.

Theorem 3.4. Under the hypotheses of Theorem 3.3, there exist $\bar{t}_{0} \in\left[t_{0}, 1\right)$ and $\bar{t}_{1} \in\left(1, t_{1}\right]$, such that for all $t \in\left[\bar{t}_{0}, \bar{t}_{1}\right]$,

$$
-\mathfrak{e}\left(m_{t}\right)=\sup _{\mu \in \mathcal{G}_{f}^{H}}\left\{h_{\mu}(f)+t \int \varphi d \mu\right\}-P_{\mathcal{M}_{f}}(t \varphi) .
$$

Moreover, the following limit defines a measure $\nu_{t}^{H}$,

$$
\nu_{t}^{H}(\psi)=\lim _{n \rightarrow \infty} e^{\mathfrak{e}\left(m_{t}\right) n} \int_{I^{n}} \psi g_{t}^{H} d m_{t}, \quad \text { for all } \psi \in \mathcal{C}^{0}(I) .
$$

The measure $\nu_{t}^{H}$ belongs to $\mathcal{G}_{f}^{H}$ and attains the supremum in (6). In addition, the limit (5) holds for any $\psi \in \mathcal{C}^{p}(I)$ with $\nu_{t}^{H}(\psi)>0$, and for $\psi g_{t}^{0}$ whenever $\nu_{t}^{H}\left(\psi g_{t}^{0}\right)>0$.

The next theorem characterizes the Hausdorff dimension of the survivor set according to the Bowen formula.

Theorem 3.5. Under the hypotheses of Theorem 3.4. $\operatorname{dim}_{H}\left(\stackrel{\circ}{ }^{\infty}\right)=\operatorname{dim}_{H}\left(\AA^{\infty}\right)=t^{*}$, where $t^{*}<1$ is the unique value of $t$ such that $\sup _{\mu \in \mathcal{G}_{f}^{H}}\left\{h_{\mu}(f)+t \int \varphi d \mu:-\mu(\varphi)<\infty\right\}=0$, i.e., such that $\mathfrak{e}\left(m_{t}\right)=P_{\mathcal{M}_{f}}(t \varphi)$.

To state our final results regarding the zero-hole limit, in what follows we will take our holes to be symmetric intervals

$$
H_{\varepsilon}=H_{\varepsilon}(z):=(z-\varepsilon, z+\varepsilon)
$$

around a point $z \in I$. To state our next result, in the case that $z$ is a periodic point, we will need to make an assumption on $z$, which we call condition

(P) The density $\frac{d \mu_{t}}{d m_{t}}$ is bounded at $z$, for the relevant $t$, and condition (31) given in Section 9 is satisfied. 
Theorem 3.6. Let $t \in\left[t_{0}, t_{1}\right], z \in I$ and suppose Crit hole $:=\{z\}$ satisfies (H2). Fix $\delta=\delta_{0}>0$ appearing in (C1)-(C2). Then there exists $h>0$ such that

$$
\frac{\mathfrak{e}\left(m_{t}, H_{\varepsilon}(z)\right)}{\mu_{t}\left(H_{\varepsilon}(z)\right)} \rightarrow \begin{cases}1 & \text { for } \mu_{t} \text {-a.e. } z, \\ 1-e^{S_{p} \phi_{t}(z)} & \text { if } z \text { is periodic of (prime) period } p \text { and }(P) \text { holds, }\end{cases}
$$

where the limit is taken as $\varepsilon \rightarrow 0$ over $H_{\varepsilon}(z) \in \mathcal{H}(h)$.

In the following result, we show that our conditions for Theorem 3.6 are met in a reasonable family of maps. Consider the logistic family $f_{\lambda}: x \mapsto \lambda x(1-x)$ on $I$. Recall that if $f_{\lambda}$ has a hyperbolic periodic cycle $\left\{z_{\lambda}, f_{\lambda}\left(z_{\lambda}\right), \ldots, f_{\lambda}^{n-1}\left(z_{\lambda}\right)\right\}$, then for all nearby $\lambda^{\prime}$, this cycle persists in the sense that there is a cycle $\left\{z_{\lambda^{\prime}}, f_{\lambda^{\prime}}\left(z_{\lambda^{\prime}}\right), \ldots, f_{\lambda^{\prime}}^{n-1}\left(z_{\lambda^{\prime}}\right)\right\}$ which depends analytically on the parameter and which converges to the original cycle as $\lambda^{\prime} \rightarrow \lambda$. This family of cycles is called the hyperbolic continuation of the original cycle.

For this family of maps, we denote by $m_{\lambda, t}$ the $\left(\varphi_{t}-p_{t}\right)$-conformal measure for $f_{\lambda}$ and by $\mu_{\lambda, t} \ll m_{\lambda, t}$ its equilibrium state, when it exists.

Theorem 3.7. There is a positive measure set of parameters $\Omega \subset(3,4]$ and an interval $\left[t_{0}, t_{1}\right] \ni 1$ such that for $t \in\left[t_{0}, t_{1}\right]$ and $\lambda \in \Omega$, the map $f_{\lambda}$ has an equilibrium state $\mu_{\lambda, t} \ll m_{\lambda, t}$, and

(a) for $\mu_{\lambda, t}$-a.e. $z \in I$

$$
\frac{\mathfrak{e}\left(m_{\lambda, t}, H_{\varepsilon}(z)\right)}{\mu_{\lambda, t}\left(H_{\varepsilon}(z)\right)} \rightarrow 1
$$

(b) if $z_{4}$ is a periodic point of (prime) period $p$ for $f_{4}$, then there exists a positive measure family of parameters $\Omega\left(z_{4}\right) \subset \Omega$ such that for $\lambda \in \Omega\left(z_{4}\right)$ and for $z_{\lambda}$ denoting the hyperbolic continuation of $z_{4}$,

$$
\frac{\mathfrak{e}\left(m_{\lambda, t}, H_{\varepsilon}\left(z_{\lambda}\right)\right)}{\mu_{\lambda, t}\left(H_{\varepsilon}\left(z_{\lambda}\right)\right)} \rightarrow 1-e^{S_{p} \phi_{t}\left(z_{\lambda}\right)} .
$$

In both limits above, (H2) is assumed to hold for Crit $t_{\text {hole }}=\{z\}$, and the limit is taken as $\varepsilon \rightarrow 0$ over holes $H_{\varepsilon}(z) \in \mathcal{H}(h)$, for $h>0$ sufficiently small.

\section{AN INDUCING SCHEME WITH UNIFORM TAIL RATES WHICH COVERS MOST OF OUR SPACE: Theorem 3.1 AND Proposition 3.2}

In this section we describe our inducing schemes and then prove that the Hausdorff dimension of points not liftable to $\Delta$ is not large: This is Theorem 3.1(a). In order to prove the remaining parts of that theorem, it is useful to have some continuity properties of Hausdorff dimension of measures and of the sets $\AA^{\infty}$. Since Proposition 3.2 gives us some of these properties we prove the proposition before completing the proof of Theorem 3.1 .

As in Section 3, we fix a set Crit hole $_{\text {and }} \delta_{0}>0$ sufficiently small such that (H2) and (2) are satisfied for $\delta=\delta_{0}$. This in turn fixes all $\delta$ appearing in $(\mathrm{C} 1)-(\mathrm{C} 2)$ to have value $\delta_{0}$. Let $\mathcal{H}(h)$ denote the family of holes defined in Section 3 .

Under these conditions, in [BDM] an interval $X \subset B_{\delta_{0}}(c)$ for some $c \in \mathrm{Crit}_{c}$ is fixed and inducing schemes $(X, F, \tau, H)$ are constructed over $X$ with uniform tails for all $H \in \mathcal{H}(h)$ : there exists $C_{1}, \alpha_{1}>0$ such that $m_{1}(\tau>n) \leqslant C_{1} e^{-\alpha_{1} n}$ where $C_{1}$ and $\alpha_{1}$ depend only on $h$ once Crit hole is fixed. Necessarily, $h \leqslant \delta_{0}$, so that $X$ is disjoint from $H$ for all $H \in \mathcal{H}(h)$. We set $\delta_{1}=m_{1}(X)$ and note that $\delta_{1}<\delta_{0}$ by the construction in [BDM]. 
4.1. Proof of Theorem 3.1(a). The proof of Theorem 3.1(a) follows [BDM, Lemma 4.5] closely (which in turn follows [DHL] rather closely).

The inducing time on the interval $X$ of length $\delta_{1}<\delta_{0}$ is constructed following a standard algorithm: $X$ is iterated forward until it is cut by either the boundary of the hole or the exponential critical partition defined in a $\delta_{0}$-neighborhood of each critical point. The resulting subintervals are then iterated, waiting for a proper return to $X$ which occurs during a 'free' period and consists of an interval which overlaps $X$ by at least $\delta_{1} / 3$ on each side. This defines the return time function $\tau$ on $X$. Intervals passing through $B_{\delta_{0}}(c)$ for some $c \in$ Crit $_{c}$ undergo a 'bound' period whose length depends on the depth of the return, which is the index of the critical partition. The definitions of 'free' and 'bound' in this context are by now standard (see [DHL, Sect. 2.2]). The only new feature created after the introduction of the hole in [BDM, Lemma 4.5] is a short bound period which allows the derivative to grow sufficiently between cuts due to $\partial H$. This avoids the problem of repeated cutting potentially destroying expansion and is formulated formally in (H2)(c).

Let $\mathcal{Q}^{(n)}$ denote the set of subintervals of $X$ induced by these subdivisions after $n$ steps which have not made a proper return by time $n$. By [BDM, Lemma 4.5], we have

$$
\sum_{\omega \in \mathcal{Q}^{(n)}}|\omega| \leqslant C_{1} e^{-\alpha_{1} n}
$$

where $|\omega|$ denotes the length of the interval $\omega$.

The set of intervals in $\mathcal{Q}^{(n)}$ form an open cover for $\mathcal{N} \mathcal{R}_{\Delta}$ for each $n$, thus we may use them to bound the Hausdorff dimension of $\mathcal{N} \mathcal{R}_{\Delta}$. First we define a coarser partition $\tilde{\mathcal{Q}}^{(n)}$ of the set $\{\tau>n\}$ by grouping intervals $\omega \in \mathcal{Q}^{(n)}$ as follows: We glue together adjacent intervals $\omega \in \mathcal{Q}^{(n)}$ which are in a bound period at time $n$ and which have not been separated by an intersection with $\partial H \cup \operatorname{Crit}_{c} \cup\left(\cup_{c \in \mathrm{Crit}_{c}} \partial B_{\delta_{0}}(c)\right)$ at any time $0 \leqslant k \leqslant n$.

We claim that the cardinality of $\tilde{\mathcal{Q}}^{(n)}$ is finite and bounded exponentially in $n$. To prove this claim, note that $\tilde{\mathcal{Q}}^{(1)}=\{X\}$ and that elements of $\tilde{\mathcal{Q}}^{(n)}$ are formed from elements $\omega \in \tilde{\mathcal{Q}}^{(n-1)}$ in one of three ways:

- $f(\omega) \cap \partial H \neq \emptyset$;

- $f(\omega) \cap\left(c \cup \partial B_{\delta_{0}}(c)\right)$ for some $c \in \mathrm{Crit}_{c}$;

- $\omega$ is bound at time $n-1$, but part of $\omega$ becomes free at time $n$.

Since $\partial H$ and $\mathrm{Crit}_{c}$ are finite, the only point we need to consider is the third.

Suppose $x$ enters a bound period in $B_{\delta_{0}}(c)$ at time $k$ with depth $r$ in the critical partition, i.e., $\left|f^{k}(x)-c\right| \approx e^{-r}$. In order for $x$ to become free again at time $n$, we must have $\left|\left(f^{n-k}\right)^{\prime}(x)\right| \geqslant$ $\kappa^{-1} e^{\theta(n-k)}$ for some constant $\theta>0$ by [DHL, Lemma 2]. By (C1), we must also have $\left|\left(f^{k}\right)^{\prime}(x)\right| \geqslant$ $\kappa e^{\gamma k}$ upon entry to $B_{\delta_{0}}(c)$. Thus $\left|\left(f^{n}\right)^{\prime}(x)\right| \geqslant \zeta$ for some constant $\zeta>1$. Now let $M=$ $\max _{x \in I}|D f(x)|$. Then since $\left|D f\left(f^{k} x\right)\right| \leqslant C e^{-\left(\ell_{c}-1\right) r}$ by definition of the critical order $\ell_{c}$ of $c$, we must have

$$
\zeta \leqslant\left|D f^{n}(x)\right| \leqslant C e^{-\left(\ell_{c}-1\right) r} M^{n} \Longrightarrow r \leq \frac{n \log M-\log (\zeta / C)}{\ell_{\max }-1} .
$$

So the number of intervals becoming free at time $n$ from a single intersection with the critical partition has a linear bound in $n$. Putting these facts together with the finiteness of $\partial H$ and Crit ${ }_{c}$ implies that the cardinality of $\tilde{\mathcal{Q}}^{(n)} \leqslant C K^{n}$ for some constants $C>0, K>1$ and all $n \in \mathbb{N}$ as required. 
Now for $s<1$, we estimate using (7),

$$
\begin{aligned}
\sum_{\tilde{\omega} \in \tilde{\mathcal{Q}}^{(n)}}|\tilde{\omega}|^{s} & \leqslant\left(\sum_{\tilde{\omega} \in \tilde{\mathcal{Q}}^{(n)}}|\tilde{\omega}|\right)^{s}\left(\# \tilde{Q}^{(n)}\right)^{1-s} \\
& =\left(\sum_{\omega \in \mathcal{Q}^{(n)}}|\omega|\right)^{s}\left(\# \tilde{Q}^{(n)}\right)^{1-s} \leqslant C_{1}^{s} e^{-\alpha_{1} n s} C^{1-s} K^{n(1-s)} .
\end{aligned}
$$

The above expression tends to 0 as $n \rightarrow \infty$ as long as $-\alpha_{1} s+(1-s) \log K<0$, i.e., as long as $s>\log K /\left(\alpha_{1}+\log K\right)$. Thus $\operatorname{dim}_{H}\left(\mathcal{N R}_{\Delta}\right) \leqslant \log K /\left(\alpha_{1}+\log K\right)=: D<1$, and Theorem 3.1(a) is proved.

4.2. Proof of Proposition 3.2. For our class of $\mathcal{C}^{2}$ maps, the inducing schemes and towers constructed in [BDM, Section 4.3] satisfy the following properties. For the distortion bounds, see also [DHL, Proposition 3].

(A1) There exist constants $\xi>1$ and $C_{d}^{\prime}>0$ such that

(a) for any $x \in X, n \geq 1$ and $k<\tau^{n}(x),\left|D f^{\tau^{n}(x)-k}\left(f^{k} x\right)\right| \geqslant\left(C_{d}^{\prime}\right)^{-1} \xi^{\tau^{n}(x)-k}$.

(b) Let $x, y \in X_{i}$ and $\tau_{i}=\tau\left(X_{i}\right)$. Then $\left|\frac{e^{S_{\ell} \varphi(x)}}{e^{S_{\ell} \varphi(y)}}\right| \leq C_{d}^{\prime}$ for $\ell \leq \tau_{i}$. In addition, $\left|\frac{e^{S \tau_{i} \varphi(x)}}{e^{S_{\tau_{i}} \varphi(y)}}-1\right| \leq C_{d}^{\prime} d\left(f^{\tau_{i}}(x), f^{\tau_{i}}(y)\right)$, or in alternative notation, $\left|\frac{e^{\Phi(x)}}{e^{\Phi(y)}}-1\right| \leqslant$ $C_{d}^{\prime} d(F(x), F(y))$, where $\Phi$ is the induced version of $\varphi$.

(A2) There exists $L<\infty$ and an index set $\mathcal{I} \subset[0, L] \times \mathbb{N}$ such that ${ }^{3}$

(a) $m_{1}\left(I \backslash \cup_{(\ell, j) \in \mathcal{I}} \pi\left(\stackrel{\circ}{\ell, j}_{\ell}\right)\right)=0$;

(b) $\pi\left({\stackrel{\circ}{\ell_{1}, j_{1}}}_{1}\right) \cap \pi\left({\stackrel{\circ}{\ell_{2}, j_{2}}}_{2}\right)=\emptyset$ for all but finitely many $\left(\ell_{1}, j_{1}\right),\left(\ell_{2}, j_{2}\right) \in \mathcal{I}$;

(c) Define $J_{1} \pi_{\ell, j}:=\left.J_{1} \pi\right|_{\dot{\Delta}_{\ell, j}}$. Then $\sup _{(\ell, j) \in \mathcal{I}}\left|J_{1} \pi_{\ell, j}\right|_{\infty}+\operatorname{Lip}\left(J_{1} \pi_{\ell, j}\right)<\infty$.

Here $J_{1} \pi$ is the Jacobian of $\pi$ with respect to Lebesgue measure $m_{1}$ and the corresponding induced measure $\bar{m}_{1}$ on $\Delta$ and Lip denotes the Lipschitz constant measured in the symbolic metric $d_{\beta}$ on $\Delta$ (both $\bar{m}_{1}$ and $d_{\beta}$ are defined in Section 5). Property (A1) guarantees expansion and bounded distortion at return times. In particular, it guarantees that the partition $\left\{\Delta_{\ell, j}\right\}$ is generating. (A2)

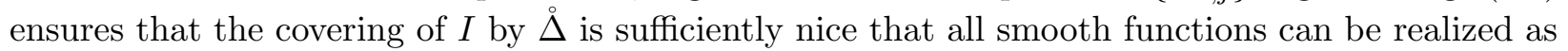
projections of Lipschitz densities from the tower [BDM, Proposition 4.2].

Lemma 4.1 (Bounded Distortion for $\phi_{t}$ ). For the potential $\phi_{t}(x):=-t \log |D f(x)|-p_{t}$, we have the induced potential $\Phi_{t}(x)-\tau(x) p_{t}=S_{\tau(x)} \varphi_{t}(x)-\tau(x) p_{t}$ for the inducing scheme. There exists $C_{d}>0$ such that for all $t \in[0,2]$ and any $x, y \in X_{i}$, for $0 \leqslant \ell \leqslant \tau_{i}$, we have $\left|\frac{e^{S_{\ell} \phi_{t}(x)}}{e^{S_{\ell} \phi_{t}(y)}}\right| \leqslant C_{d}$. Moreover,

$$
\left|\frac{e^{\Phi_{t}(x)-\tau(x) p_{t}}}{e^{\Phi_{t}(y)-\tau(y) p_{t}}}-1\right| \leqslant C_{d} d(F(x), F(y)) .
$$

Furthermore, $\Phi_{t}-\tau p_{t}$ is locally Hölder.

\footnotetext{
${ }^{3}$ See [BDM, Lemma 4.6] for a proof of property (A2).
} 
Proof. Since $e^{S_{\ell} \phi_{t}(x)}=e^{t S_{\ell} \varphi(x)-\ell p_{t}}$ and $e^{S_{\ell} \phi_{t}(y)}=e^{t S_{\ell} \varphi(y)-\ell p_{t}}$, (A1)(b) implies immediately that $\left|\frac{e^{S_{\ell} \phi_{t}(x)}}{e^{S_{\ell} \phi_{t}(y)}}\right| \leqslant\left(C_{d}^{\prime}\right)^{t}$. For the second equation, it is equivalent to estimate

$$
\log \left|\frac{e^{S_{\tau_{i}} \phi_{t}(x)}}{e^{S_{\tau_{i}} \phi_{t}(y)}}\right| \leqslant t \log \left|\frac{e^{S_{\tau_{i}} \varphi(x)}}{e^{S_{\tau_{i}} \varphi(y)}}\right|
$$

so this part of the lemma follows from $(\mathrm{A} 1)(\mathrm{b})$ as long as $t$ is in a compact interval. This implies that $V_{1}\left(\Phi_{t}\right)$ is bounded. The fact that $V_{n}\left(\Phi_{t}\right)$, and hence $V_{n}\left(\Phi_{t}-\tau p_{t}\right)$, decays exponentially in $n$ follows similarly, but now also using (A1)(a) to show that if $x, y$ are in the same $n$-cylinder, then $d(F(x), F(y))$ is exponentially small in $n$.

Remark 4.2. We have already stated that for the potential $\varphi_{t}(x):=-t \log |D f(x)|$, we have the induced potential $\Phi_{t}(x):=S_{\tau(x)} \varphi_{t}(x)$ for the inducing scheme. Now we also denote the corresponding punctured potentials by $\varphi_{t}^{H}$ and $\Phi_{t}^{H}$. We often denote $\Phi_{1}$, the induced potential corresponding to $\varphi$, by $\Phi$. Observe that since our inducing schemes respect the hole, any measure on the survivor set cannot give mass to any column $\cup_{\ell=0}^{\tau_{i}-1} \Delta_{i, \ell}$ with a hole in it. Therefore, for the purposes of computing pressure, we can either think of $\Phi_{t}^{H}$ on the corresponding base element $X_{i}$ as being $-\infty$, or think of $X_{i}$ as not being part of the inducing scheme. In either case, the Hölder property of the variations persists for the punctured potentials.

The tail estimates in [BDM] hold for the inducing schemes with respect to Lebesgue measure. We will show that we have related tail rates for the conformal measures corresponding to $\Phi_{t}$ for all $t$ sufficiently close to 1 and for all small holes.

Define

$$
\begin{aligned}
\chi_{M} & :=\sup \left\{\log \left|D f^{n}(x)\right| / n: f^{n}(x)=x\right\} \text { and } \\
\chi_{m} & :=\inf \left\{\log \left|D f^{n}(x)\right| / n: f^{n}(x)=x\right\} .
\end{aligned}
$$

By [BS], (C2) implies that $\chi_{m}>0$. Moreover, for a measure $\mu \in \mathcal{M}_{f}$, let

$$
\chi(\mu):=\int \log |D f| d \mu .
$$

Each interval $X_{i}$ contains a point $x_{i}$ such that $f^{\tau_{i}}\left(x_{i}\right)=x_{i}$. So by the bounded distortion of Lemma 4.1 and the definitions of $\chi_{m}$ and $\chi_{M}$, we have $-\chi_{M} \tau_{i} \lesssim S_{\tau_{i}} \varphi\left(y_{i}\right)=\Phi\left(y_{i}\right) \lesssim-\chi_{m} \tau_{i}$, for any $y_{i} \in X_{i}$, where $\lesssim$ denotes a uniform constant depending only on the distortion. Now $S_{\tau} \phi_{t}=t S_{\tau} \varphi-\tau p_{t}$. Therefore, choosing $y_{i}^{\prime} \in X_{i}$ such that $e^{S_{\tau_{i}} \varphi\left(y_{i}^{\prime}\right)}=m_{1}\left(X_{i}\right) / m_{1}(X)$, we obtain for $0<t<1$,

$$
\sum_{\tau_{i}=n} e^{S_{\tau} \phi_{t}\left(y_{i}\right)} \leqslant C_{d} e^{n\left[(1-t) \chi_{M}-p_{t}\right]} \sum_{\tau_{i}=n} e^{S_{\tau_{i}} \varphi\left(y_{i}^{\prime}\right)} \leqslant C_{1} C_{d} \delta_{1}^{-1} e^{n\left[(1-t) \chi_{M}-p_{t}\right]} e^{-\alpha_{1} n} .
$$

Now since $p_{1}=0$ and $p_{t}$ is a continuous function of $t$ (see for example [IT1]), we may choose $t_{0}^{\prime}<1$ such that $(1-t) \chi_{M}-p_{t}-\alpha_{1}<0$ for all $t \in\left[t_{0}^{\prime}, 1\right]$.

The proof for $t>1$ follows similarly with $\chi_{m}$ in place of $\chi_{M}$. In this case, the constraint is $-(t-1) \chi_{m}-p_{t}-\alpha_{1}<0$ for all $t \in\left[1, t_{1}^{\prime}\right]$, with the restriction on $t_{1}^{\prime}>1$ coming from the fact that $p_{t}<0$. Then define

$$
\alpha:=\alpha_{1}-\max \left\{\sup _{t \in\left[t_{0}^{\prime}, 1\right]}(1-t) \chi_{M}-p_{t}, \sup _{t \in\left[1, t_{1}^{\prime}\right]}(1-t) \chi_{m}-p_{t}\right\} .
$$


By conformality and bounded distortion, we have $m_{t}\left(X_{i}\right)=m_{t}(X) e^{S_{\tau} \phi_{t}\left(y_{i}\right)}$ for some $y_{i} \in X_{i}$ and each $i$, which completes the proof of Proposition 3.2 .

Note that in order for Proposition 3.2 and Theorem 3.1 to be coherent, we will choose the interval $\left[t_{0}, t_{1}\right]$ in the statement of the Proposition 3.2 to be contained in $\left[t_{0}^{\prime}, t_{1}^{\prime}\right]$ chosen above in order that the Hausdorff dimension considerations discussed in Section 4.1 hold.

4.3. Proof of parts (b) $-(\mathbf{d})$ of Theorem 3.1. For this proof we will repeatedly make use of the fact, proved in [Ho, that if $\mu \in \mathcal{M}_{f}$ then $\operatorname{dim}_{H}(\mu)=h(\mu) / \chi(\mu)$.

For part (b), we write $\stackrel{\circ}{ }^{\infty} \subset \pi\left(\AA^{\infty}\right) \cup \mathcal{N} \mathcal{R}_{\Delta}$ and by Theorem 3.1 (a), we have $\operatorname{dim}_{H}\left(\mathcal{N} \mathcal{R}_{\Delta}\right) \leqslant D<$ 1. Note that the invariant measure $\nu_{1}^{H}$ corresponding to the potential $\varphi_{1}$ from [BDM] satisfies $\log \lambda_{1}^{H}=h\left(\nu_{1}^{H}\right)-\chi\left(\nu_{1}^{H}\right)$, so that $\operatorname{dim}_{H}\left(\nu_{1}^{H}\right)=\frac{h\left(\nu_{1}^{H}\right)}{\chi\left(\nu_{1}^{H}\right)}=1+\log \lambda_{1}^{H}$. Moreover, in [BDM] it is shown that $\log \lambda_{1}^{H} \rightarrow 0$ as the hole shrinks to zero, so $\operatorname{dim}_{H}\left(\nu_{1}^{H}\right)$ can be made arbitrarily close to 1 (and $>D)$ for any $H \in \mathcal{H}(h)$ with $h$ sufficiently small. This implies that $\operatorname{dim}_{H}\left(\pi\left(\AA^{\infty}\right)\right)>D$ so that necessarily, $\operatorname{dim}_{H}\left(\stackrel{\circ}{I}^{\infty}\right)=\operatorname{dim}_{H}\left(\stackrel{\circ}{\Delta}^{\infty}\right)$.

As before, let $\mu_{t}$ denote the equilibrium state for $\varphi_{t}$ (before the introduction of the hole). For (c), we have $\operatorname{dim}_{H}\left(m_{t}\right)=\operatorname{dim}_{H}\left(\mu_{t}\right)=\frac{h\left(\mu_{t}\right)}{\chi\left(\mu_{t}\right)}=t+\frac{P_{\mathcal{M}_{f}}\left(\varphi_{t}\right)}{\chi\left(\mu_{t}\right)}$. As in [IT1, this value is continuous in $t$, so $\operatorname{dim}_{H}\left(m_{t}\right)$ is close to 1 for $t$ close enough to 1 since $P_{\mathcal{M}_{f}}\left(\varphi_{1}\right)=0$. So part (a) implies that $m_{t}\left(\mathcal{N} \mathcal{R}_{\Delta}\right)=0$. We set $t_{1}^{\prime} \geqslant t_{1}>1$ and $t_{0}^{\prime} \leqslant t_{0}<1$ to be such that this holds for all $t \in\left[t_{0}, t_{1}\right]$.

For (d), we will use the fact that $\chi_{m}>0$. Part (a) implies that a measure $\mu \in \mathcal{M}_{f}^{H}$ which doesn't lift to $\left(\Delta, f_{\Delta}\right)$ must have $\operatorname{dim}_{H}(\mu)=h(\mu) / \chi(\mu)<D$. Thus

$$
h(\mu)+t \int \varphi^{H} d \mu=h(\mu)-t \chi(\mu)<-(t-D) \chi(\mu) \leqslant-(t-D) \chi_{m} .
$$

Since for $t$ sufficiently close to 1 and $H$ sufficiently small, $P_{\Delta}\left(\varphi_{t}^{H}\right)$ is approximately 0 , the result follows. Note that this holds since $P_{\Delta}\left(\varphi_{1}^{H}\right)$ is continuous in the size of the hole by [BDM] and $P_{\Delta}\left(\varphi_{t}^{H}\right)$ is continuous in $t$.

\section{Review of Known Results: Transfer Operator on the Tower with Holes}

We recall the abstract setup of [BDM] into which we shall place our induced maps in order to prove Theorems $3.3-3.6$.

Let $f_{\Delta}: \Delta \circlearrowleft$ be a Young tower formed over an inducing scheme $(f, X, \tau)$ as described in Section 2.5 Given a $\phi$-conformal reference measure $m$ on $I$, we define a reference measure $\bar{m}$ on $\Delta$ by $\bar{m}=m$ on $\Delta_{0}=X$ and $\left.\bar{m}\right|_{\Delta_{\ell}}=\left.\left(f_{\Delta}\right)_{*} \bar{m}\right|_{\Delta_{\ell-1} \cap f_{\Delta}^{-1} \Delta_{\ell}}$ for $\ell \geqslant 1$. For $x \in \Delta_{\ell}$, let $x^{-}:=f^{-\ell} x$ denote the pullback of $x$ to $\Delta_{0}$. We define the induced potential on $\Delta$ by,

$$
\phi_{\Delta}(x)=S_{\tau} \phi\left(x^{-}\right) \text {for } x \in f_{\Delta}^{-1}\left(\Delta_{0}\right) \text { and } \phi_{\Delta}=0 \text { on } \Delta \backslash f_{\Delta}^{-1}\left(\Delta_{0}\right) .
$$

With these definitions, the measure $\bar{m}$ is $\phi_{\Delta}$-conformal. As in Section 2.5, we assume that the partition $\left\{\Delta_{\ell, j}\right\}$ (equivalently $\left\{X_{i}\right\}$ ) is generating and that all returns to $\Delta_{0}$ satisfy $f_{\Delta}^{\tau}\left(\Delta_{\ell, j}\right)=\Delta_{0} !^{4}$ We assume that the tower has exponential returns:

\footnotetext{
4 The abstract setup in BDM] uses the more general finite images condition, but since the towers constructed in [BDM] actually satisfy full returns to a single base, we will use this simpler version here.
} 
(P1) There exist constants $C, \alpha>0$ such that $\bar{m}\left(\Delta_{n}\right) \leqslant C e^{-\alpha n}$, for $n \in \mathbb{N}$ (this is equivalent to saying that $\left.m(\tau=n)=O\left(e^{-\alpha n}\right)\right)$.

The tower inherits a natural metric adapted to the dynamics as follows. Define the separation time on $\Delta$ to be

$$
s(x, y)=\min \left\{n \geq 0: f_{\Delta}^{n} x, f_{\Delta}^{n} y \text { lie in different partition elements } \Delta_{\ell, j}\right\} .
$$

$s(x, y)$ is finite $\mu$-almost everywhere for any $\mu$ that lifts to the tower since $\left\{X_{i}\right\}$ is a generating partition for $f^{\tau}$. Choose $\beta \in(0, \alpha)$ and define a metric $d_{\beta}$ on $\Delta$ by $d_{\beta}(x, y)=e^{-\beta s(x, y)}$.

We introduce a hole $H$ in $\Delta$ which is the union of countably many partition elements $\Delta_{\ell, j}$, i.e., $H=\cup_{\ell, k} H_{\ell, k}$ where $H_{\ell, k}=\Delta_{\ell, j}$ for some $j$. Set $H_{\ell}=\cup_{j} H_{\ell, j} \subset \Delta_{\ell}$. For simplicity we assume that the base $\Delta_{0}$ contains no holes (this can always be arranged in the construction of the tower by choosing a suitable reference set $X$ ). We assume the following additional properties for the tower.

(P2) (Bounded Distortion) We suppose that $\phi_{\Delta}$ is Lipshitz in the metric $d_{\beta}$. Furthermore, we assume there exists $C_{d}>0$ such that for all $x, y \in \Delta$ and $n \geq 0$,

$$
\left|e^{S_{n} \phi_{\Delta}(x)-S_{n} \phi_{\Delta}(y)}-1\right| \leqslant C_{d} d_{\beta}\left(f_{\Delta}^{n} x, f_{\Delta}^{n} y\right) .
$$

(P3) (Subexponential growth of potential) For each $\varepsilon>0$, there exists $C>0$, such that

$$
\left|S_{\tau} \phi_{\Delta}\right| \leqslant C e^{\varepsilon \tau} \text { for all return times } \tau \text {. }
$$

(P4) (Smallness of the hole) Let $H_{\ell}=\cup_{j} H_{\ell, j}$ and set $q:=\sum_{\ell \geqslant 1} \bar{m}\left(H_{\ell}\right) e^{\beta(\ell-1)}$. We assume

$$
q<\frac{\left(1-e^{-\beta}\right) \bar{m}\left(\Delta_{0}\right)}{1+C_{d}} .
$$

We say the open system is mixing if g.c.d. $\left\{\left.\tau\right|_{\Delta_{0} \cap f_{\Delta}^{-\tau}\left(\Delta_{0}\right)}\right\}=1$ and $\stackrel{\circ}{f}_{\Delta}$ still admits at least one return to $\Delta_{0}$.

Following Section 2.4, we define the transfer operator $\mathcal{L}_{\phi_{\Delta}^{H}}$ associated with the punctured potential $\phi_{\Delta}^{H}$ and acting on $L^{1}(\bar{m})$ by

$$
\mathcal{L}_{\phi_{\Delta}^{H}}^{n} \psi(x)=\sum_{f_{\Delta}^{n} y=x} \psi(x) e^{S_{n} \phi_{\Delta}(y)} 1_{\check{\Delta}^{n}}(y)=\mathcal{L}_{\phi_{\Delta}}^{n}\left(\psi 1_{\check{\Delta}^{n}}\right)(x)
$$

where $\stackrel{\circ}{ }^{n}=\cap_{i=0}^{n} f_{\Delta}^{-i} \stackrel{\circ}{\Delta}$ as before. For notational simplicity, we will denote $\mathcal{L}_{\phi_{\Delta}^{H}}$ simply by $\dot{\mathcal{L}}$ for the remainder of this section since the potential $\phi_{\Delta}$ is fixed. When we wish to vary the potential in later sections, we will reintroduce subscripts to reinforce the explicit dependence on the potential and the hole.

5.1. A Spectral Gap for the Transfer Operator. We define the following function spaces on $\Delta$ used in $[\mathrm{Y}, \mathrm{BDM}]$ on which the transfer operator $\stackrel{\circ}{\mathcal{L}}$ for the tower with a hole has a spectral gap.

For $\psi \in L^{1}(\bar{m})$, define

$$
\begin{aligned}
\left\|\psi_{\ell, j}\right\|_{\text {Lip }} & =e^{-\beta \ell} \operatorname{Lip}\left(\left.\psi\right|_{\Delta_{\ell, j}}\right) \\
\left\|\psi_{\ell, j}\right\|_{\infty} & =e^{-\beta \ell} \sup _{\Delta_{\ell, j}}|\psi|
\end{aligned}
$$

Then define $\|\psi\|_{\text {Lip }}=\sup _{\ell, j}\left\|\psi_{\ell, j}\right\|_{\text {Lip }},\|\psi\|_{\infty}=\sup _{\ell, j}\left\|\psi_{\ell, j}\right\|_{\infty}$, and $\|\psi\|_{\mathcal{B}}=\|\psi\|_{\text {Lip }}+\|\psi\|_{\infty}$. 
Note that if $\|\psi\|_{\infty}<\infty$, then

$$
\int_{\Delta} \psi d \bar{m} \leq\|\psi\|_{\infty} \sum_{\ell} e^{\beta \ell} \bar{m}\left(\Delta_{\ell}\right) \leqslant\|\psi\|_{\infty} \sum_{\ell} e^{\beta \ell} m(\tau \geq \ell)<\infty
$$

by choice of $\beta$ so that $\psi \in L^{1}(\bar{m})$. Let $\mathcal{B}=\mathcal{B}_{\beta}:=\left\{\psi \in L^{1}(\bar{m}):\|\psi\|_{\mathcal{B}}<\infty\right\}$.

The following theorem is proved in $[\mathrm{BDM}]$.

Theorem 5.1. ([BDM]) Let $\left(f_{\Delta}, \Delta, H\right)$ satisfy (P1)-(P4) and assume the open system is mixing. Then $\mathcal{L}$ has essential spectral radius bounded by $e^{-\beta}$ and spectral radius given by $e^{-\beta}<\lambda<1$. The eigenvalue $\lambda$ is simple and all other eigenvalues have modulus strictly less than 1.

Let $g \in \mathcal{B}$ denote the unique probability density corresponding to $\lambda$. Then there exists $\sigma<1$ and $C>0$ such that for all $\psi \in \mathcal{B}$,

(i) $\left\|\lambda^{-n} \dot{\mathcal{L}}^{n} \psi-c(\psi) g\right\|_{\mathcal{B}} \leqslant C\|\psi\|_{\mathcal{B}} \sigma^{n}$, where $c(\psi)$ is a constant depending on $\psi$;

(ii) moreover, $c(\psi)>0$ if and only if $\lim _{n \rightarrow \infty}\left\|\mathcal{L}^{n} \psi /\left|\dot{L}^{n} \psi\right|_{1}-g\right\|_{\mathcal{B}}=0$, where convergence is at the rate $\sigma^{n}$.

The primary object of interest in open systems is the limit in (ii) above since it describes the class of densities whose escape rate matches that of $g$. Thus it raises the question, for which functions is $c(\psi)>0$ ? We characterize this set more precisely in the next section by constructing an invariant measure on the survivor set, which will also serve as an equilibrium state for the open system.

5.2. Construction of equilibrium state. We begin with what is by now a standard construction of an invariant measure on the survivor set $\stackrel{\Delta}{\Delta}^{\infty}:=\cap_{n=0}^{\infty} f_{\Delta}^{-n} \stackrel{\circ}{\Delta}$. Let $\mathcal{B}_{0}$ denote the space of functions $\mathcal{B}_{\beta}$, but with $\beta=0$.

Note that by the conditional invariance equation $\stackrel{\circ}{\mathcal{L}} g=\lambda g$, and the fact that $g \geq \varepsilon>0$ on $\Delta_{0}$ ([BDM, Proposition 2.4]), we have $C \lambda^{-\ell} \leqslant\left. g\right|_{\Delta_{\ell}} \leqslant C^{-1} \lambda^{-\ell}$, for some constant $C>0$ depending only on (P1)-(P4). Since $\lambda>e^{-\beta}$ by construction, it follows from the definitions of $\mathcal{B}$ and $\mathcal{B}_{0}$ that $g \psi \in \mathcal{B}$ whenever $\psi \in \mathcal{B}_{0}$.

Take $\psi \in \mathcal{B}_{0}$. Since $g \psi \in \mathcal{B}$, by Theorem 5.1(i), we may define,

$$
\mathcal{Q}(\psi):=\lim _{n \rightarrow \infty} \lambda^{-n} g^{-1} \stackrel{\mathcal{L}}{ }^{n}(g \psi)=c(g \psi) .
$$

This defines a linear functional on $\mathcal{B}_{0}$. We also have $\left|\mathcal{L}^{n}(g \psi)\right| \leq|\psi|_{\infty} \dot{\mathcal{L}}^{n} g=|\psi|_{\infty} \lambda^{n} g$, so that $|\mathcal{Q}(\psi)| \leq|\psi|_{\infty}$ and $\mathcal{Q}$ is a bounded, linear functional on $\mathcal{B}_{0}$. By the Riesz representation theorem, there exists a measure $\tilde{\nu}$ such that $\mathcal{Q}(\psi)=\tilde{\nu}(\psi)$ for all $\psi \in \mathcal{B}_{0}$. Since $\mathcal{Q}(1)=1$, it follows that $\tilde{\nu}$ is a probability measure. Indeed, it is easy to check that $\tilde{\nu}$ is an invariant probability measure for $f_{\Delta}$ supported on $\stackrel{\circ}{\Delta}^{\infty}$.

It follows from $\mathrm{BDM}$, Sect. 3.3] that $\tilde{\nu}$ is ergodic and enjoys exponential decay of correlations on functions in $\mathcal{B}_{0}$.

[BDM, Section 3.4] uses $\tilde{\nu}$ to formulate the following proposition regarding convergence and escape rates.

Proposition 5.2. ([BDM]) Let $\left(f_{\Delta}, \Delta, \bar{m}, H\right)$ be as in the statement of Theorem 5.1. 
(i) For each $\psi \in \mathcal{B}_{0}$ with $\psi \geqslant 0$, we have $\tilde{\nu}(\psi)>0$ if and only if

$$
\lim _{n \rightarrow \infty} \frac{{\stackrel{\circ}{\mathcal{L}^{n}} \psi}^{\left|\dot{\mathcal{L}}^{n} \psi\right|_{1}}}{\mid{ }^{n}}=g,
$$

where, as usual, the convergence is at an exponential rate in the $\|\cdot\|$-norm. In particular, the reference measure converges to the conditionally invariant measure $g d \bar{m}$.

(ii) Let $\psi \in \mathcal{B}, \psi \geq 0$, with $\tilde{\nu}(x: \psi(x)>0)>0$. Then the limit in (i) holds. Moreover, the escape rate with respect to the measure $\psi \bar{m}$ exists and equals $-\log \lambda$.

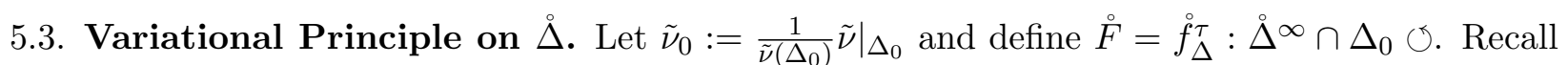
that $\tau^{n}(x)=\sum_{k=0}^{n-1} \tau\left(\stackrel{\circ}{F}^{k} x\right)$ denotes the $n^{\text {th }}$ return time starting at $x$ and let $\mathcal{M}_{\dot{F}}$ be the set of $\stackrel{\circ}{F}$-invariant Borel probability measures on $\stackrel{\circ}{ }^{\infty} \cap \Delta_{0}$. We will need the following two lemmas, the first of which is proved in [BDM, Lemma 5.3].

Lemma 5.3. Let $X_{n, i} \subset \Delta_{0}$ denote a cylinder set of length $n$ with respect to $\stackrel{\circ}{F}$. Then there exists a constant $C>0$ such that for all $n$,

$$
C^{-1} \lambda^{-\tau^{n}\left(y_{*}\right)} e^{S_{\tau^{n} \phi_{\Delta}\left(y_{*}\right)}} \leq \tilde{\nu}\left(X_{n, i}\right) \leq C \lambda^{-\tau^{n}\left(y_{*}\right)} e^{S_{\tau^{n} \phi_{\Delta}}\left(y_{*}\right)}
$$

where $y_{*}$ is an arbitrary point in $X_{n, i}$.

The following lemma is missing from [BDM] and is added here as a correction in the abstract setting (see also [DWY] for a similar correction).

Lemma 5.4. The measure $\tilde{\nu}_{0}$ satisfies $\tilde{\nu}_{0}\left(-S_{\tau} \phi_{\Delta}\right)<\infty$ and $\tilde{\nu}(\tau)<\infty$.

Proof. That $\tilde{\nu}_{0}(\tau)<\infty$ is trivial since $\tilde{\nu}_{0}$ is a restriction of $\tilde{\nu}$ and $\tilde{\nu}(\Delta)=1$ :

$$
\int_{\Delta_{0}} \tau d \tilde{\nu}_{0}=\left(\tilde{\nu}\left(\Delta_{0}\right)\right)^{-1} \sum_{n} n \tilde{\nu}(\tau=n)=\left(\tilde{\nu}\left(\Delta_{0}\right)\right)^{-1} \tilde{\nu}(\Delta)<\infty .
$$

To show that $\tilde{\nu}_{0}\left(-S_{\tau} \phi_{\Delta}\right)<\infty$, we use the bounds given by Lemma 5.3 as well as assumption (P3). Note that by definition of conformal measure, we have

$$
e^{S_{\tau} \phi_{\Delta}\left(y_{i}\right)}=\frac{\bar{m}\left(X_{i}\right)}{\bar{m}\left(\Delta_{0}\right)}
$$

for some $y_{i} \in X_{i}$ and each $i$. Choosing $\varepsilon<\alpha-\beta$ in 11 and setting $c_{0}=\left(\tilde{\nu}\left(\Delta_{0}\right)\right)^{-1}$, we write

$$
\begin{aligned}
\int_{\Delta_{0}}-S_{\tau} \phi_{\Delta} d \tilde{\nu}_{0} & \leq\left. c_{0} \sum_{i}\left|S_{\tau} \phi_{\Delta}\right|_{X_{i}}\right|_{\infty} \tilde{\nu}\left(X_{i}\right) \leq C \sum_{i} e^{\varepsilon \tau\left(X_{i}\right)} \lambda^{-\tau\left(X_{i}\right)} e^{S_{\tau} \phi_{\Delta}\left(y_{i}\right)} \\
& \leq C^{\prime} \sum_{n} e^{\varepsilon n} \lambda^{-n} \bar{m}(\tau=n) \leqslant C^{\prime \prime} \sum_{n} e^{-(\alpha+\log \lambda-\varepsilon) n}
\end{aligned}
$$

Recall that $\lambda>e^{-\beta}$ so that $\log \lambda>-\beta$. Thus the exponent in the sum above is greater than $\alpha-\beta-\varepsilon>0$ by choice of $\varepsilon$, and so the series converges.

Lemmas 5.3 and 5.4 imply that $\tilde{\nu}_{0}$ is a Gibbs measure with respect to the potential $S_{\tau} \phi_{\Delta}-\tau \log \lambda$.

Notice that for $x \in \Delta_{0}, S_{\tau} \phi_{\Delta}(x)=\sum_{i=0}^{\tau(x)-1} \phi_{\Delta}\left(f_{\Delta}^{i} x\right)$. However, $\phi_{\Delta}\left(f_{\Delta}^{i} x\right)=0$ for $i<\tau(x)-1$, so that $S_{\tau} \phi_{\Delta}(x)=\phi_{\Delta}\left(f_{\Delta}^{\tau-1} x\right)$. Using this, for $\eta_{0} \in \mathcal{M}_{F}^{\circ}$, we have

$$
\int_{\Delta_{0}} S_{\tau} \phi_{\Delta} d \eta_{0}=\eta\left(\Delta_{0}\right)^{-1} \int_{f_{\Delta}^{-1} \Delta_{0}} \phi_{\Delta} d \eta=\eta\left(\Delta_{0}\right)^{-1} \int_{\Delta} \phi_{\Delta} d \eta
$$


so that $\eta\left(-\phi_{\Delta}\right)<\infty$ if and only if $\eta_{0}\left(-S_{\tau} \phi_{\Delta}\right)<\infty$. Thus there is a 1-1 correspondence between the relevant measures in $\mathcal{M}_{F}^{\circ}$ and $\mathcal{M}_{f_{\Delta}}^{H}$, the set of ergodic, $f_{\Delta}$ invariant probability measures supported on $\stackrel{\circ}{ }^{\infty}$. This implies in particular that $\tilde{\nu}\left(-\phi_{\Delta}\right)<\infty$ by Lemma 5.4 so that $\tilde{\nu} \in \mathcal{M}_{f_{\Delta}}^{H}$. This leads to the following equilibrium principle for $f_{\Delta}$.

Proposition 5.5. ([BDM, Theorem 2.9]) Suppose $\psi \in \mathcal{B}, \psi \geq 0$, satisfies $\tilde{\nu}(\psi)>0$ and $\int \psi d \bar{m}=$ 1. Let $\mathfrak{e}\left(\bar{m}_{\psi}\right)$ be the escape rate of $\bar{m}_{\psi}:=\psi \bar{m}$ from $\stackrel{\Delta}{\Delta}$. Then

$$
-\mathfrak{e}\left(\bar{m}_{\psi}\right)=\log \lambda=\sup _{\eta \in \mathcal{M}_{f_{\Delta}}^{H}}\left\{h_{\eta}\left(f_{\Delta}\right)+\int_{\Delta} \phi_{\Delta} d \eta:-\int_{\Delta} \phi_{\Delta} d \eta<\infty\right\} .
$$

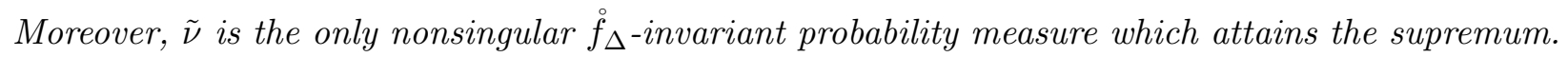

\section{Proof of Theorem 3.3}

In this section, we return to our specific class of maps described in Sect. 2 and use the results of Section 5 to obtain conditionally invariant measures absolutely continuous with respect to the $\left(\varphi_{t}-p_{t}\right)$-conformal measures $m_{t}$, where $\varphi_{t}=-t \log |D f|$ and $p_{t}=P_{\mathcal{M}_{f}}\left(\varphi_{t}\right)$. In order to invoke the results of Section 5, we first verify properties (P1)-(P4) of the constructed towers.

To distinguish between holes in $I$ and $\Delta$, we shall denote by $H$ the hole in $I$ and by $\tilde{H}=\pi^{-1} H$ the hole in $\Delta$. Thus for consistency, $\tilde{H}=\cup_{\ell \geqslant 1} \tilde{H}_{\ell}$ and $\tilde{H}_{\ell}=\cup_{j} \tilde{H}_{\ell, j}$.

6.1. (P1)-(P4) are satisfied with uniform constants. We fix Crit hole $_{\text {and }} \delta_{0}>0$ as in Section 4 . Then for $H \in \mathcal{H}(h)$ with $h$ sufficiently small, by [BDM] we have an inducing scheme and Young tower satisfying properties (A1) and (A2). Let $\bar{m}_{t}$ denote the reference measure on $\Delta$ induced by $m_{t}$, the $\phi_{t}$-conformal measure. Recall that by Theorem 3.1, this measure is guaranteed to lift to $\Delta$ if $t \in\left[t_{0}, t_{1}\right]$.

By Proposition 3.2, we have (P1) satisfied uniformly with respect to $\bar{m}_{t}$ for some $\alpha>0$ (the same $\alpha$ as in Proposition 3.2 and all $t \in\left[t_{0}, t_{1}\right]$. We choose $\beta \in(0, \alpha)$ and add the restriction that $\beta \leqslant t_{0} \log \xi$ (see the proof of Lemma 6.2). Then (P2) follows from Lemma 4.1 and (A1)(a) with a possibly larger constant $C_{d}$ for the potentials $\phi_{\Delta, t}$ induced by $\phi_{t}=\varphi_{t}-p_{t}$. (P3) is automatic for our class of maps since $|D f|$ is bounded above and due to (A1)(a), we have $\left|S_{\tau}\left(\phi_{t}\right)\right| \leqslant C \tau$ at return times. Again, all constants are uniform for $t \in\left[t_{0}, t_{1}\right]$.

It remains to verify $(\mathrm{P} 4)$ for the constructed towers. We do this via the following lemma.

Lemma 6.1. There exists $h>0$ sufficiently small such that if $H \in \mathcal{H}(h)$ then (P4) is satisfied with respect to the measure $\bar{m}_{t}$ for all $t \in\left[t_{0}, t_{1}\right]$.

Proof. We need to show,

$$
\sum_{\ell \geqslant 1} \frac{\bar{m}_{t}\left(\tilde{H}_{\ell}\right)}{\bar{m}_{t}\left(\Delta_{0}\right)} e^{\beta(\ell-1)}<\frac{1-e^{-\beta}}{1+C_{d}}
$$


First assume that $t \in\left[t_{0}, 1\right]$. Recall that each component $\tilde{H}_{\ell, j} \subset \Delta$ is a 1 -cylinder for the tower map $f_{\Delta}$. We have for some $y \in \tilde{H}_{\ell, j}$,

$$
\begin{aligned}
\frac{\bar{m}_{t}\left(\tilde{H}_{\ell, j}\right)}{\bar{m}_{t}\left(\Delta_{0}\right)} & =e^{S_{\tau} \phi_{\Delta, t}(y)}=e^{t S_{\tau} \varphi_{\Delta}(y)-\tau(y) p_{t}} \\
& =e^{(t-1) S_{\tau} \varphi_{\Delta}(y)-\tau(y) p_{t}} e^{S_{\tau} \varphi_{\Delta}(y)} \\
& \leqslant C_{d} e^{\tau\left(f^{-\ell} \tilde{H}_{\ell, j}\right)\left[(1-t) \chi_{M}-p_{t}\right]} \frac{\bar{m}_{1}\left(\tilde{H}_{\ell, j}\right)}{\bar{m}_{1}\left(\Delta_{0}\right)}
\end{aligned}
$$

where $\chi_{M}$ is as in (8). We use this to estimate (16),

$$
\sum_{\ell \geqslant 1} \frac{\bar{m}_{t}\left(\tilde{H}_{\ell}\right)}{\bar{m}_{t}\left(\Delta_{0}\right)} e^{\beta(\ell-1)} \leqslant C_{d} \delta_{1}^{-1} \sum_{\ell \geqslant 1} \sum_{j} e^{\beta(\ell-1)} e^{\tau\left(f^{-\ell} \tilde{H}_{\ell, j}\right)\left[(1-t) \chi_{M}-p_{t}\right]} \bar{m}_{1}\left(\tilde{H}_{\ell, j}\right),
$$

where $\delta_{1}=m_{1}(X)$.

Note that $f_{\Delta}^{-\ell} \tilde{H}_{\ell, j}$ is a 1-cylinder for the induced map $F: X \rightarrow X$. Set

$$
b_{t}=(1-t) \chi_{M}-p_{t} \text { and } A_{n}=\left\{\tilde{H}_{\ell, j}: \tau\left(f_{\Delta}^{-\ell} \tilde{H}_{\ell, j}\right)=n\right\} .
$$

Then since $\ell \leqslant \tau\left(f^{-\ell}\left(\tilde{H}_{\ell, j}\right)\right)$, our estimate becomes,

$$
\sum_{\ell \geqslant 1} \frac{\bar{m}_{t}\left(\tilde{H}_{\ell}\right)}{\bar{m}_{t}\left(\Delta_{0}\right)} e^{\beta(\ell-1)} \leqslant C \sum_{n \geqslant 1} \sum_{\tilde{H}_{\ell, j} \in A_{n}} e^{\beta \ell} e^{n b_{t}} \bar{m}_{1}\left(\tilde{H}_{\ell, j}\right) \leqslant C \sum_{n \geqslant 1} e^{\left(\beta+b_{t}-\alpha_{1}\right) n},
$$

since $A_{n} \subset\{\tau=n\}$. Note that $\alpha_{1}-b_{t} \geqslant \alpha$ and $\beta<\alpha$ by choice of $\left[t_{0}, t_{1}\right]$ and $\beta$ so that the sum is uniformly bounded for $t$ in this interval.

In order to show that the sum can in fact be made arbitrarily small, we split it into two parts, depending on whether $\tilde{H}_{\ell, j}$ is created by an intersection of $f^{\ell}(X)$ with $H$ during a free period or during a bound period. Thus

$$
\sum_{\ell, j} e^{\beta \ell} e^{\tau_{\ell, j} b_{t}} \bar{m}_{1}\left(\tilde{H}_{\ell, j}\right)=\sum_{\text {bound }} e^{\beta \ell} e^{\tau_{\ell, j} b_{t}} \bar{m}_{1}\left(\tilde{H}_{\ell, j}\right)+\sum_{\text {free }} e^{\beta \ell} e^{\tau_{\ell, j} b_{t}} \bar{m}_{1}\left(\tilde{H}_{\ell, j}\right),
$$

where $\tau_{\ell, j}=\tau\left(f_{\Delta}^{-\ell} \tilde{H}_{\ell, j}\right)$.

To estimate the sum over bound pieces, we use the slow approach condition (H1). Suppose $\omega \subset X$ is a 1-cylinder in $X$ such that $f^{n}(\omega) \subset H$ during a bound period, $n<\tau(\omega)$, and $c \in \mathrm{Crit}_{c}$ is the last critical point visited by $\omega$ at time $n-\ell$. Since $\omega$ is bound, we have $\left|f^{\ell} x-f^{\ell} c\right| \leqslant \delta_{0} e^{-2 \vartheta_{c} \ell}$ for all $x \in f^{n-\ell} \omega$ by [DHL, Sect. 2.2]. This implies that $\operatorname{dist}\left(f^{\ell} c, \partial H\right) \leqslant m_{1}(H)+\delta_{0} e^{-2 \vartheta_{c} \ell}$. On the other hand, (H1) requires $\operatorname{dist}\left(f^{\ell} c, \partial H\right) \geqslant \delta_{0} e^{-\vartheta_{c} \ell}$. This forces,

$$
\delta_{0} e^{-\vartheta_{c} \ell} \leqslant m_{1}(H)+\delta_{0} e^{-2 \vartheta_{c} \ell} \Longrightarrow \ell \geqslant-\vartheta_{c}^{-1} \log \left(h / \delta_{c}\right),
$$

where $\delta_{c}=\delta_{0}\left(1-e^{-\vartheta_{c}}\right)$. Thus since $\ell \leqslant \tau_{\ell, j}$,

$$
\begin{aligned}
\sum_{\text {bound }} e^{\beta \ell} e^{\tau_{\ell, j} b_{t}} \bar{m}_{1}\left(\tilde{H}_{\ell, j}\right) & \leqslant \sum_{\text {bound }} e^{\left(\beta+b_{t}\right) \tau_{\ell, j}} \bar{m}_{1}\left(\tilde{H}_{\ell, j}\right) \\
& \leqslant \sum_{n>-\vartheta_{c}^{-1} \log \left(h / \delta_{c}\right)} C e^{\left(\beta+b_{t}-\alpha_{1}\right) n} \leqslant C^{\prime} h^{\vartheta_{c}^{-1}(\alpha-\beta)} .
\end{aligned}
$$


To estimate the sum over free pieces, we use the following estimate from BDM, Proof of Lemma $4.5]$,

$$
\sum_{\text {free }} \bar{m}_{1}\left(\tilde{H}_{\ell, j}\right) \leqslant C m_{1}(H)
$$

Then

$$
\begin{aligned}
\sum_{\text {free }} e^{\beta \ell} e^{\tau_{\ell, j} b_{t}} \bar{m}_{1}\left(\tilde{H}_{\ell, j}\right) & \leqslant \sum_{\tau_{\ell, j} \leqslant-\log h} e^{\left(\beta+b_{t}\right) \tau_{\ell, j}} \bar{m}_{1}\left(\tilde{H}_{\ell, j}\right)+\sum_{\tau_{\ell, j}>-\log h} e^{\left(\beta+b_{t}\right) \tau_{\ell, j} \bar{m}_{1}\left(\tilde{H}_{\ell, j}\right)} \\
& \leqslant e^{-\left(\beta+b_{t}\right) \log h} \sum_{\tau_{\ell, j} \leqslant-\log h} \bar{m}_{1}\left(\tilde{H}_{\ell, j}\right)+\sum_{\tau_{\ell, j}>-\log h} e^{\left(\beta+b_{t}-\alpha_{1}\right) \tau_{\ell, j}} \\
& \leqslant C h^{1-\left(\beta+b_{t}\right)}+h^{\alpha_{1}-\beta-b_{t}},
\end{aligned}
$$

where all exponents are positive due to the choice of $\beta$ and $t_{0}$.

The argument for $t \in\left[1, t_{1}\right]$ is similar with $b_{t}$ defined by $(1-t) \chi_{m}-p_{t}$.

6.2. Pushing forward densities on $I$. We have proved that (P1)-(P4) hold with uniform constants for all $t \in\left[t_{0}, t_{1}\right]$ and all $H \in \mathcal{H}(h)$ for $h$ sufficiently small. We now fix such an $H \in \mathcal{H}(h)$. By Theorem 5.1, for each $t$, we have a conditionally invariant density $\tilde{g}_{t}^{H} \in \mathcal{B}$ satisfying $\dot{\mathcal{L}}_{\phi_{\Delta, t}} \tilde{g}_{t}^{H}=$ $\lambda_{t}^{H} \tilde{g}_{t}^{H}$, where $\lambda_{t}^{H}<1$ is a simple eigenvalue of $\stackrel{\circ}{\mathcal{L}}_{\phi_{\Delta, t}}$ with maximum modulus. We use the spectral

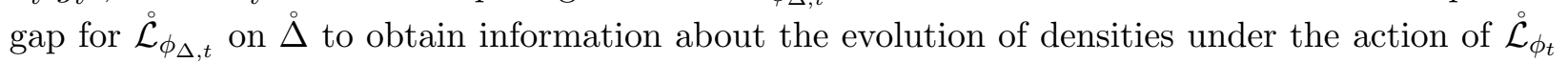
on $I$.

The philosophy is the following. For $\tilde{\psi} \in \mathcal{B}$, let

$$
\mathcal{P}_{\pi, t} \tilde{\psi}(x)=\sum_{y \in \pi^{-1} x} \frac{\tilde{\psi}(y)}{J_{t} \pi(y)}
$$

where $J_{t} \pi$ is the Jacobian of $\pi$ with respect to the measures $m_{t}$ and $\bar{m}_{t}$. The commuting relation $f^{n} \circ \pi=\pi \circ f_{\Delta}^{n}$ implies

$$
\mathcal{P}_{\pi, t}\left(\stackrel{\circ}{\mathcal{L}}_{\phi \Delta, t}^{n} \tilde{\psi}\right)=\stackrel{\circ}{\mathcal{L}}_{\phi_{t}}^{n}\left(\mathcal{P}_{\pi, t} \tilde{\psi}\right)
$$

so that the evolution of densities on $I$ under $\stackrel{\circ}{\mathcal{L}}_{\phi_{t}}$ matches the evolution of densities on $\Delta$ under $\stackrel{\circ}{\mathcal{L}}_{\phi_{\Delta, t}}$ for those densities in $\mathcal{P}_{\pi, t} \mathcal{B}$. Indeed, $\left|\mathcal{P}_{\pi, t} \tilde{\psi}\right|_{L^{1}\left(m_{t}\right)}=|\tilde{\psi}|_{L^{1}\left(\bar{m}_{t}\right)}$ so that mass is preserved.

The question of which densities on $I$ can be realized as projections of elements of $\mathcal{B}$ (or $\mathcal{B}_{0}$ ) is addressed in $[\mathrm{BDM}]$ and is somewhat subtle and system dependent. Given $\psi \in \mathcal{C}^{p}(I)$, we define $\tilde{\psi}=\psi \circ \pi$ and it is a consequence of (A1) that $\tilde{\psi} \in \mathcal{B}_{0}$ for all $p \geqslant \beta / \log \xi$, where $\xi>1$ is from (A1)(a) BDM, Lemma 4.1]. However, in general, $\mathcal{P}_{\pi, t}(\psi \circ \pi) \neq \psi$ so that this is not sufficient to characterize those densities which may be realized as projections from of elements in $\mathcal{B}$.

Note that this requirement is different from the problem of liftability of the measure $\psi m_{t}$. For an invariant measure $\mu$, if $\mu$ lifts to an invariant measure $\tilde{\mu}$ on $\Delta$, then $\pi_{*} \tilde{\mu}=\mu$ as described in Section 2.6. but for a density with respect to $m_{t}$, this may not be the case since in general, $\pi_{*} \bar{m}_{t} \neq m_{t}$, even for $t=1$. In order to proceed, we will need the following lemma, which is essentially a version of property (A2) with respect to the measures $m_{t}$.

Lemma 6.2. Let $\mathcal{I} \subset[0, L] \times \mathbb{N}$ be as in (A2). Then for all $t \in\left[t_{0}, t_{1}\right]$,

(a) $m_{t}\left(I \backslash \cup_{(\ell, j) \in \mathcal{I}} \pi\left(\stackrel{\circ}{\ell, j}_{\ell, j}\right)\right)=0$

(b) $\pi\left({\stackrel{\circ}{\ell_{1}, j_{1}}}_{1}\right) \cap \pi\left({\stackrel{\circ}{\ell_{2}, j_{2}}}_{2}\right)=\emptyset$ for all but finitely many $\left(\ell_{1}, j_{1}\right),\left(\ell_{2}, j_{2}\right) \in \mathcal{I}$;

(c) Define $J_{t} \pi_{\ell, j}:=\left.J_{t} \pi\right|_{\dot{\Delta}_{\ell, j}}$. Then $\sup _{(\ell, j) \in \mathcal{I}}\left|J_{t} \pi_{\ell, j}\right|_{\infty}+\operatorname{Lip}\left(J_{t} \pi_{\ell, j}\right)<\infty$. 
As a consequence, $\mathcal{C}^{p}(I) \subset \mathcal{P}_{\pi, t}\left(\mathcal{B}_{0}\right)$ for all $p \geqslant \beta / \log \xi$, where $\xi>1$ is from (A1).

Proof. To prove (a), recall that if we ignore cuts due to the countable exponential partition of $B_{\delta_{0}}(c)$ for each $c \in \mathrm{Crit}_{c}$, then $\pi\left(\Delta_{\ell}\right)$ consists of finitely many intervals. Thus according to the proof of (A2)(a) in BDM, Lemma 4.6], $I \backslash \cup_{(\ell, j) \in \mathcal{I}} \pi\left(\stackrel{\circ}{\ell, j}_{\ell}\right)$ contains at most the endpoints of these finitely many intervals together with the images of the cuts of the exponential partition. This set is countable and so its $m_{t}$ measure is zero.

Item (b) is independent of the measure and so is trivially true by (A2)(b).

It remains to prove (c). For $x \in \Delta_{\ell}$, let $x_{-\ell}=f_{\Delta}^{-\ell} x \in \Delta_{0}$. Then by conformality and the definition of $\bar{m}_{t}$, we have

$$
J_{t} \pi(x)=\frac{d m_{t}(\pi x)}{d \bar{m}_{t}(x)}=e^{-S_{\ell} \phi_{t}\left(x_{-\ell}\right)}=e^{-t S_{\ell} \varphi\left(x_{-\ell}\right)+\ell p_{t}}=\left(J_{1} \pi(x)\right)^{t} e^{\ell p_{t}} .
$$

Since $\ell \leqslant L$ by definition of $\mathcal{I}$ and due to property (A2)(c) of $J_{1} \pi$, the above relation implies the required bound on the $L^{\infty}$-norm of $J_{t} \pi$ restricted to elements of $\mathcal{I}$.

To prove the bound on the Lipschitz constant of $J_{t} \pi$, we restrict our attention to the case $t \in\left[t_{0}, 1\right)$ since for $t \geqslant 1$, the Lipschitz property of $J_{t} \pi$ follows from that of $J_{1} \pi$. Now using the fact that $\left|a^{t}-b^{t}\right| \leqslant|a-b|^{t}$ for $t<1$, we use (18) to estimate for $x, y \in \Delta_{\ell, j}$,

$$
\left|J_{t} \pi(x)-J_{t} \pi(y)\right| \leqslant\left|J_{1} \pi(x)-J_{1} \pi(y)\right|^{t} e^{\ell p_{t}} \leqslant\left|D f^{\ell}\left(\pi\left(x_{-\ell}\right)\right)-D f^{\ell}\left(\pi\left(y_{-\ell}\right)\right)\right|^{t} e^{\ell p_{t}} .
$$

Since $\ell \leqslant L$ and $f$ is $\mathcal{C}^{2}$, this bound yields,

$$
\left|J_{t} \pi(x)-J_{t} \pi(y)\right| \leqslant C\left|\pi\left(x_{-\ell}\right)-\pi\left(y_{-\ell}\right)\right|^{t} .
$$

Let $s_{0}=s\left(x_{-\ell}, y_{-\ell}\right)$. Since $s_{0}$ is a return time for $x_{-\ell}, y_{-\ell}$, we have $\left|D f^{s_{0}}\right| \geqslant\left(C_{d}^{\prime}\right)^{-1} \xi^{s_{0}}$ by (A1)(a). Thus

$$
\left|\pi\left(x_{-\ell}\right)-\pi\left(y_{-\ell}\right)\right| \leqslant C_{d}^{\prime} \xi^{-s_{0}}\left|f^{s_{0}}\left(\pi x_{-\ell}\right)-f^{s_{0}}\left(\pi y_{-\ell}\right)\right| \leqslant C_{d}^{\prime} \xi^{-s_{0}} \operatorname{diam}(X) .
$$

Putting this together with 190 yields

$$
\left|J_{t} \pi(x)-J_{t} \pi(y)\right| \leqslant C \xi^{-t s_{0}} \leqslant C e^{-\beta s_{0}} \leqslant C d_{\beta}(x, y)
$$

since $s(x, y)=s_{0}-\ell$ and as long as $\xi^{-t} \leqslant e^{-\beta}$, which is true for $t \geqslant t_{0}$ by choice of $\beta \leqslant t_{0} \log \xi$. This completes the proof of (c).

Now using properties (a)-(c) for $J_{t} \pi$, it follows from [BDM, Proposition 4.2] that $\mathcal{C}^{p}(I) \subset \mathcal{P}_{\pi, t}\left(\mathcal{B}_{0}\right)$ for all $p \geqslant \beta / \log \xi$.

For $p \geqslant \beta / \log \xi$, define $\mathcal{D}^{p}(I)$ to be the set of nonnegative functions $\psi \in \mathcal{C}^{p}(I)$ with $\psi>0$ on $X$. The following proposition completes the proof of Theorem 3.3 .

Proposition 6.3. Let $\tilde{\mu}_{t}^{H}=\tilde{g}_{t}^{H} \bar{m}_{t}$ and define $\pi_{*} \tilde{\mu}_{t}^{H}=\mu_{t}^{H}=\left(\mathcal{P}_{\pi, t} \tilde{g}_{t}^{H}\right) m_{t}$. Then $\mu_{t}^{H}$ is a conditionally invariant measure for $f$ with eigenvalue $\lambda_{t}^{H}$. In addition,

(i) For all $\psi \in \mathcal{D}^{p}(I)$,

$$
\lim _{n \rightarrow \infty} \frac{\dot{\mathcal{L}}_{\phi_{t}}^{n} \psi}{\left|\dot{\mathcal{L}}_{\phi_{t}}^{n} \psi\right|_{1}}=\mathcal{P}_{\pi, t} \tilde{g}_{t}^{H} \quad \text { in } L^{1}\left(m_{t}\right)
$$

and the convergence occurs at an exponential rate so that $\mu_{t}^{H}$ is a geometric conditionally invariant measure, absolutely continuous with respect to $m_{t}$.

(ii) Let $\psi \in \mathcal{D}^{p}(I)$. The escape rate with respect to the reference measure $\psi m_{t}$ is given by

$$
\mathfrak{e}\left(\psi m_{t}\right)=-\log \lambda_{t}^{H}
$$


(iii) Let $\mu_{t}$ be the equilibrium state for the potential $\phi_{t}=\varphi_{t}-p_{t}$ before the introduction of the hole. Then for all $\psi \in \mathcal{D}^{p}(I), \mathfrak{e}\left(\psi \mu_{t}\right)=\mathfrak{e}\left(m_{t}\right)=-\log \lambda_{t}^{H}$ and

$$
\lim _{n \rightarrow \infty} \frac{\stackrel{\circ}{*}_{*}^{n}\left(\psi \mu_{t}\right)}{\left|\stackrel{\circ}{*}_{*}^{n}\left(\psi \mu_{t}\right)\right|}=\mu_{t}^{H}
$$

Proof. The fact that $\pi_{*} \tilde{\mu}_{t}^{H}$ defines a conditionally invariant measure with the same eigenvalue as $\tilde{\mu}_{t}^{H}$ follows from the relation $\pi \circ \stackrel{\circ}{\Delta}_{\Delta}=\stackrel{\circ}{\circ} \circ \pi$.

(i) Suppose $\psi \in \mathcal{D}^{p}(I)$. By Lemma 6.2 , we may define $\bar{\psi} \in \mathcal{B}_{0}$ such that $\mathcal{P}_{\pi, t} \bar{\psi}=\psi$. Then $\tilde{\nu}_{t}(\bar{\psi})>0$ since $\psi \geqslant 0$ and $\psi>0$ on $X$ (indeed, this is trivial since we may always take $X$ to be among the set of elements specified by (A2) to cover $I$ ). Then, by Proposition 5.2 (i),

$$
\frac{\stackrel{\circ}{\mathcal{L}}_{\phi t}^{n} \psi}{\left|\dot{\mathcal{L}}_{\phi_{t}}^{n} \psi\right|_{1}}=\frac{\mathcal{P}_{\pi, t} \stackrel{\circ}{\mathcal{L}}_{\phi_{\Delta, t}}^{n} \bar{\psi}}{\left|\dot{\mathcal{L}}_{\phi_{\Delta, t}}^{n} \bar{\psi}\right|_{1}} \stackrel{n \rightarrow \infty}{\longrightarrow} \mathcal{P}_{\pi, t} \tilde{g}_{t}^{H},
$$

in the $L^{1}\left(m_{t}\right)$ norm where we have used the fact that

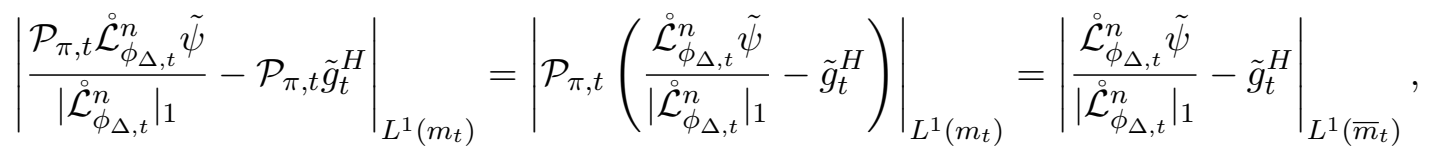

and the convergence is at an exponential rate since the $\|\cdot\|_{\mathcal{B}}$-norm dominates the $L^{1}\left(\bar{m}_{t}\right)$ norm and $\stackrel{\circ}{\mathcal{L}}_{\phi_{\Delta, t}}$ has a spectral gap on $\mathcal{B}$.

(ii) This follows from Proposition 5.2(iii) since

$$
\int_{I^{n}} \psi d m_{t}=\int_{\dot{I}} \stackrel{\circ}{\mathcal{L}}_{\phi_{t}}^{n} \psi d m_{t}=\int_{\dot{\Sigma}}{\stackrel{\circ}{\mathcal{L}_{\phi}^{n}}}_{\phi_{\Delta, t}} \bar{\psi} d \bar{m}_{t}=\int_{\dot{\Delta}^{n}} \bar{\psi} d \bar{m}_{t} .
$$

(iii) We claim that the measure $\mu_{t}=g_{t}^{0} m_{t}$ can be realized as the projection of an element in $\mathcal{B}_{0}$. Consider the tower $\Delta$ before the introduction of the hole. The arguments of Section 5 hold in the case when $H=\emptyset$ so that $\mathcal{L}_{\phi \Delta, t}$ has leading eigenvalue 1 with eigenvector $\tilde{g}_{t}^{0} \in \mathcal{B}_{0}$ which defines an invariant measure $\tilde{\mu}_{t}=\tilde{g}_{t}^{0} \bar{m}_{t}$. Then $\pi_{*} \tilde{\mu}_{t}=\mu_{t}$ and $\mathcal{P}_{\pi, t} \tilde{g}_{t}^{0}=g_{t}^{0}$, proving the claim. Since $\tilde{g}_{t}^{0}>0$ on $\Delta_{0}$, we have $\tilde{\nu}_{t}\left(\tilde{g}_{t}^{0}\right)>0$ so that $g_{t}^{0}$ is in the class of densities for which the relations in (i) and (ii) hold by Proposition 5.2 (although it is discontinuous on $I$ ).

It follows that $\psi g_{t}^{0}$ can also be realized as the projection of the element $\psi \circ \pi \cdot \tilde{g}_{t}^{0} \in \mathcal{B}_{0}$ for any $\psi \in \mathcal{C}^{p}(I)$. If in addition, $\psi \in \mathcal{D}^{p}(I)$, then $\tilde{\nu}_{t}\left(\psi \circ \pi \cdot \tilde{g}_{t}^{0}\right)>0$ so that again, the required limits hold.

Remark 6.4. As can be seen from the proof of Proposition 6.3, the convergence result (i) and escape rate (ii) hold for any $\psi \in L^{1}\left(m_{t}\right)$ which can be realized as an element of $\mathcal{P}_{\pi, t}\left(\mathcal{B}_{0}\right)$ and satisfies $\psi>0$ on $X$. In fact, this second condition can be relaxed to $\nu_{t}^{H}(\psi)>0$ once the equilibrium measure $\nu_{t}^{H}$ of Theorem 3.4 is introduced.

\section{Proof of Theorem 3.4}

As verified during the proof of Theorem 3.3, for $H \in \mathcal{H}(h)$ and $h$ sufficiently small, we have a tower $\left(f_{\Delta}, \Delta, \tilde{H}\right)$ respecting the hole, i.e., such that $\pi^{-1} H=\tilde{H}$ is a union of partition elements $\Delta_{\ell, j}$, which satisfies $(\mathrm{P} 1)-(\mathrm{P} 4)$ with uniform constants for all $t \in\left[t_{0}, t_{1}\right]$. 
Fix $H \in \mathcal{H}(h)$. We have an invariant measure $\tilde{\nu}_{t}$ supported on $\AA^{\infty}$ which satisfies the equilibrium principle of Proposition 5.5 and is defined by

$$
\tilde{\nu}_{t}(\tilde{\psi})=\lim _{n \rightarrow \infty}\left(\lambda_{t}^{H}\right)^{-n} \int_{\Delta^{n}} \tilde{\psi} \tilde{g}_{t}^{H} d \bar{m}_{t}
$$

where $\tilde{g}_{t}^{H}$ and $\lambda_{t}^{H}$ are from Theorem 5.1 .

Defining $\nu_{t}=\pi_{*} \tilde{\nu}_{t}$, we have $\nu_{t}$ supported on $\stackrel{\circ}{I}^{\infty}$ since $\pi\left(\stackrel{\circ}{\Delta}^{\infty}\right) \subset \stackrel{\circ}{I}^{\infty}$. Moreover, $\nu_{t}$ is an invariant measure for $f$ by the relation $f \circ \pi=\pi \circ f_{\Delta}$.

For $\psi \in \mathcal{C}^{0}(I)$, define $\tilde{\psi}=\psi \circ \pi$. Then

$$
\begin{aligned}
\nu_{t}(\psi) & =\tilde{\nu}_{t}(\tilde{\psi})=\lim _{n \rightarrow \infty}\left(\lambda_{t}^{H}\right)^{-n} \int_{\dot{\Delta}^{n}} \tilde{\psi} \tilde{g}_{t}^{H} d \bar{m}_{t}=\lim _{n \rightarrow \infty}\left(\lambda_{t}^{H}\right)^{-n} \int_{I^{n}} \mathcal{P}_{\pi, t}\left(\psi \circ \pi \cdot \tilde{g}_{t}^{H}\right) d m_{t} \\
& =\lim _{n \rightarrow \infty} e^{n \mathfrak{e}\left(m_{t}\right)} \int_{\tilde{I}^{n}} \psi \mathcal{P}_{\pi, t}\left(\tilde{g}_{t}^{H}\right) d m_{t},
\end{aligned}
$$

so that $\nu_{t}$ satisfies the definition of $\nu_{t}^{H}$ as defined in the limit given in Theorem 3.4 .

The convergence of $\mathcal{L}_{\phi_{t}^{H}}^{n} \psi /\left|\mathcal{L}_{\phi_{t}^{H}}^{n} \psi\right|_{L^{1}\left(m_{t}\right)}$ (respectively, $\left.\mathcal{L}_{\phi_{t}^{H}}^{n}\left(\psi g_{t}^{0}\right) /\left|\mathcal{L}_{\phi_{t}^{H}}^{n}\left(\psi g_{t}^{0}\right)\right|_{L^{1}\left(m_{t}\right)}\right)$ to $g_{t}^{H}:=\mathcal{P}_{\pi, t}\left(\tilde{g}_{t}^{H}\right)$ for any $\psi \in \mathcal{C}^{p}(I)$ with $\nu_{t}(\psi)>0$ (respectively, $\nu_{t}\left(\psi g_{t}^{0}\right)>0$ ), follows from Proposition 5.2 given that both $\psi$ and $\psi g_{t}^{0}$ can be realized as elements of $\mathcal{P}_{\pi, t}\left(\mathcal{B}_{0}\right)$ as in the proof of Proposition 6.3.

Finally, we need to show that $\nu_{t}$ achieves the supremum in the required variational principle. We begin by projecting the Variational Principle of Proposition 5.5 down to $I$.

Lemma 7.1. Let $\eta \in \mathcal{M}_{f}^{H}$ be such that $\eta$ lifts to $\Delta$ and $-\infty<\eta(-\varphi)<\infty$. Let $\tilde{\eta} \in \mathcal{M}_{f_{\Delta}}^{H}$ denote the lift of $\eta$ to $\Delta$. Then $\int_{I} \log J f d \eta=\int_{\Delta} \log J f_{\Delta} d \tilde{\eta}$, where $J f=|D f|$ and $J f_{\Delta}$ is the Jacobian of $f_{\Delta}$ with respect to $\bar{m}_{1}$.

Proof. Due to the relation $\pi \circ f_{\Delta}=f \circ \pi$, we have for $x \in \Delta$,

$$
J_{1} \pi\left(f_{\Delta} x\right) J f_{\Delta}(x)=J f(\pi x) J_{1} \pi(x) .
$$

Thus since $\pi_{*} \tilde{\eta}=\eta$,

$$
\int_{I} \log J f d \eta=\int_{\Delta} \log J f \circ \pi d \tilde{\eta}=\int_{\Delta}\left(\log J f_{\Delta}+\log J_{1} \pi \circ f_{\Delta}-\log J_{1} \pi\right) d \tilde{\eta} .
$$

We claim that $\int_{\Delta}\left(\log J_{1} \pi \circ f_{\Delta}-\log J_{1} \pi\right) d \tilde{\eta}=0$. Note that if $\int_{\Delta} \log J_{1} \pi d \tilde{\eta}$ were finite, this would be trivial by the invariance of $\tilde{\eta}$, but we do not assume the finiteness of this integral.

We consider two cases. If $x \in \Delta_{\ell} \cap f_{\Delta}^{-1}\left(\Delta_{0}\right)$, then $J_{1} \pi\left(f_{\Delta} x\right)=1$. Setting $x_{-\ell}=f_{\Delta}^{-\ell} x$ as before and using (18), we obtain

$$
\log J_{1} \pi\left(f_{\Delta} x\right)-\log J_{1} \pi(x)=S_{\ell} \varphi\left(\pi\left(x_{-\ell}\right)\right) .
$$

On the other hand, if $x \in \Delta_{\ell} \backslash f_{\Delta}^{-1}\left(\Delta_{0}\right)$, then again by (18),

$$
\log J_{1} \pi\left(f_{\Delta} x\right)-\log J_{1} \pi(x)=S_{\ell} \varphi\left(\pi\left(x_{-\ell}\right)\right)-S_{\ell+1} \varphi\left(\pi\left(x_{-\ell}\right)\right)=-\varphi(\pi x) .
$$

Putting these two cases together, we have

$$
\int_{\Delta}\left(\log J_{1} \pi \circ f_{\Delta}-\log J_{1} \pi\right) d \tilde{\eta}=\int_{f_{\Delta}^{-1}\left(\Delta_{0}\right)} S_{\ell} \varphi\left(\pi\left(x_{-\ell}\right)\right) d \tilde{\eta}-\int_{\Delta \backslash f_{\Delta}^{-1}\left(\Delta_{0}\right)} \varphi(\pi x) d \tilde{\eta} .
$$

Both integrals are finite by assumption on $\tilde{\eta}$. We decompose $\Delta$ into columns $\left\{f_{\Delta}^{\ell}\left(X_{i}\right)\right\}_{\ell<\tau\left(X_{i}\right)}$ and note that the first integral considers $S_{\ell} \varphi\left(\pi\left(x_{-\ell}\right)\right)$ in the top element of the column while the second considers the sum of $\varphi \circ \pi$ in all the levels below the top one, which is precisely the same thing. This, plus the fact that $\tilde{\eta}\left(f_{\Delta}^{\ell}\left(X_{i}\right)\right)=\tilde{\eta}\left(X_{i}\right)$ for $\ell<\tau\left(X_{i}\right)$ provides the required cancellation. 
Taking $\eta \in \mathcal{M}_{f}^{H}$ with $-\infty<\eta(-\varphi)<\infty$, we use Lemma 7.1 to write $\eta\left(\phi_{t}\right)=\tilde{\eta}\left(\phi_{\Delta, t}\right)$ for each $t$ since $\eta\left(-\phi_{t}\right)<\infty$ if and only if $\eta(-\varphi)<\infty$.

Moreover, $h_{\tilde{\eta}}\left(f_{\Delta}\right)=h_{\eta}(f)$ since $\pi$ is at most countable-to-one [B, Proposition 2.8]. Putting these together yields by Proposition 5.5 ,

$$
-\mathfrak{e}\left(m_{t}\right)=\log \lambda_{t}^{H}=\sup _{\eta \in \mathcal{M}_{f}^{H}}\left\{h_{\eta}(f)+t \int_{I} \varphi d \eta: \eta \text { lifts to } \Delta\right\}-P_{\mathcal{M}_{f}}(t \varphi) .
$$

However, the condition " $\eta$ lifts to $\Delta$ " does not suffice to prove Theorem 3.4 since that condition is not well understood and depends on the inducing scheme. In order to replace the above class of measures with the class $\mathcal{G}_{f}^{H}$ which is independent of the inducing scheme, we must prove two things:

(i) $\nu_{t} \in \mathcal{G}_{f}^{H}$; and

(ii) $-\mathfrak{e}\left(m_{t}\right) \geqslant P_{\mathcal{G}_{f}^{H}}(t \varphi)-P_{\mathcal{M}_{f}}(t \varphi)$.

Proving (i) will imply $-\mathfrak{e}\left(m_{t}\right) \leqslant P_{\mathcal{G}_{f}^{H}}(t \varphi)-P_{\mathcal{M}_{f}}(t \varphi)$ since we know $\nu_{t}$ lifts to $\Delta$ and $\nu_{t}\left(-\phi_{t}\right)<\infty$ by Lemma 5.4 and 15 . Then (ii) will yield the required equality. We proceed to prove these points in the next two subsections.

7.1. The weight near the boundary of the hole. In this section, we prove the following proposition.

Proposition 7.2. There exist $C, r>0$ such that $\nu_{t}\left(N_{\varepsilon}\left(\partial H \cup C r i t_{c}\right)\right) \leqslant C \varepsilon^{r}$ for all $\varepsilon>0$, where $N_{\varepsilon}(\cdot)$ denotes the $\epsilon$-neighborhood of a set.

Proof. Denote by $\mathcal{Z}_{n}$ the partition of $\Delta_{0}$ into $n$-cylinders for $F=f^{\tau}$. Recall that $\nu_{t}=\pi_{*} \tilde{\nu}_{t}$ and that $\tilde{\nu}_{0}:=\left.\left(\tilde{\nu}_{t}\left(\Delta_{0}\right)\right)^{-1} \tilde{\nu}_{t}\right|_{\Delta_{0}}$ is a Gibbs measure for $\stackrel{\circ}{F}$ by Lemma 5.3 which satisfies

$$
C^{-1} \lambda_{t}^{-\tau^{n}\left(y_{*}\right)} e^{S_{\tau^{n} \phi_{\Delta, t}\left(y_{*}\right)}} \leq \tilde{\nu}_{0}\left(Z_{n}\right) \leq C \lambda_{t}^{-\tau^{n}\left(y_{*}\right)} e^{S_{\tau^{n} \phi_{\Delta, t}\left(y_{*}\right)}}
$$

for any $Z_{n} \in \mathcal{Z}_{n}$ and $y^{*} \in Z_{n}$. Here $\lambda_{t}=\lambda_{t}^{H}$; we have retained the explicit dependence on $t$, but have suppressed dependence on $H$.

Fix $\varepsilon>0$ and choose $n_{0} \in \mathbb{N}$ to be the minimal $n$ such that

$$
\sup _{Z_{n} \in \mathcal{Z}_{n}} \sup _{\ell<\tau\left(Z_{n}\right)} m_{1}\left(\pi\left(f_{\Delta}^{\ell} Z_{n}\right)\right)<\varepsilon
$$

where $m_{1}$ denotes Lebesgue measure as usual. Note that such an $n_{0}$ exists due to the fact that there is exponential expansion at return times by property (A1)(a). Indeed, let $Z_{n-1}^{\prime}=F\left(Z_{n}\right)$ be the $n-1$ cylinder mapped to by $Z_{n}$. Then $m_{1}\left(\pi\left(f_{\Delta}^{\ell} Z_{n}\right)\right) \leqslant m_{1}\left(\pi Z_{n-1}^{\prime}\right) \leqslant C_{0}^{-1} \xi^{-\tau^{n-1}\left(Z_{n-1}^{\prime}\right)} m_{1}\left(\Delta_{0}\right)$, for each $\ell<\tau\left(Z_{n}\right)$. Thus $m_{1}\left(\pi\left(f_{\Delta}^{\ell} Z_{n}\right)\right) \leqslant \varepsilon$ whenever

$$
\tau^{n-1}\left(Z_{n-1}^{\prime}\right)>\frac{-\log \left(C_{0} \varepsilon / \delta_{1}\right)}{\log \xi} .
$$

Since $\tau_{n} \geq \tau_{\min } n$, where $\tau_{\min }$ denotes the minimum return time, it suffices to choose

$$
n_{0}>1+\frac{-\log \left(C_{0} \varepsilon / \delta_{1}\right)}{\tau_{\min } \log \xi} .
$$


For brevity, set $B=\partial H \cup \mathrm{Crit}_{c}$, the singularity set. Let $\mathfrak{C}_{\varepsilon}$ denote the collection of $n$-cylinders $Z_{n}$ of minimal index $n \leqslant n_{0}$ such that $\pi\left(f_{\Delta}^{\ell} Z_{n}\right) \cap N_{\varepsilon}(B) \neq \emptyset$ for some $\ell<\tau\left(Z_{n}\right)$. By minimal index, we mean that if $Z_{n}$ is contained in an $(n-1)$-cylinder $Z_{n-1}$ such that $\pi\left(f_{\Delta}^{\ell} Z_{n-1}\right) \subset N_{2 \varepsilon}(B)$, then we omit $Z_{n}$ from $\mathfrak{C}_{\varepsilon}$ and include $Z_{n-1}$ instead. For each $n \leqslant n_{0}$, define $\mathfrak{C}_{\varepsilon, n}$ to be the set of $n$-cylinders in $\mathfrak{C}_{\varepsilon}$.

Note that

$$
\nu_{t}\left(N_{\varepsilon}(B)\right) \leqslant \sum_{Z_{n} \in \mathfrak{C}_{\varepsilon}} \sum_{\text {relevant } \ell} \tilde{\nu}_{t}\left(f_{\Delta}^{\ell} Z_{n}\right)
$$

For $Z_{n} \in \mathfrak{C}_{\varepsilon}$, there are two possibilities when $\pi\left(f_{\Delta}^{\ell} Z_{n}\right) \cap N_{\varepsilon}(B) \neq \emptyset$ : either the interval $\pi\left(f_{\Delta}^{\ell} Z_{n}\right)$ is free or it is bound. If it is bound at time $\ell$ due to passing through $B_{\delta_{0}}$ (Crit) at time $\ell-k$, we have $\left|f^{k} x-f^{k} c\right| \leqslant \delta_{0} e^{-2 \vartheta_{c} k}$ for all $x \in \pi\left(f_{\Delta}^{\ell-k} Z_{n}\right)$ and some $c \in$ Crit $_{c}$ by [DHL, Sect. 2.2], where $\vartheta_{c}$ is from (C2). This implies that $\operatorname{dist}\left(f^{k} c, B\right) \leqslant \varepsilon+\delta_{0} e^{-2 \vartheta_{c} k}$. On the other hand, the slow approach conditions (C2) and (H1) imply that $\operatorname{dist}\left(f^{k} c, B\right) \geq \delta_{0} e^{-\vartheta_{c} k}$. Putting these two conditions together, we must have

$$
\delta_{0} e^{-2 \vartheta_{c} k}-\delta_{0} e^{-\vartheta_{c} k}+\varepsilon \geq 0
$$

which admits two possibilities: either

$$
e^{-\vartheta_{c} k}<\frac{1-\sqrt{1-\frac{4 \varepsilon}{\delta_{0}}}}{2} \quad \text { or } \quad e^{-\vartheta_{c} k}>\frac{1+\sqrt{1-\frac{4 \varepsilon}{\delta_{0}}}}{2} .
$$

Since $k \geq 1$ by (H1), we may eliminate the second possibility by only considering $\varepsilon$ sufficiently small that $e^{-\vartheta_{c}}<\frac{1+\sqrt{1-\frac{4 \varepsilon}{\delta_{0}}}}{2}$. For the first possibility to occur, we estimate $\sqrt{1-x} \geqslant 1-x$, for $0 \leqslant x \leqslant 1$, and solve for $k$ to obtain the requirement

$$
k>\frac{-\log \left(2 \varepsilon / \delta_{0}\right)}{\vartheta_{c}} .
$$

Thus we must have $\tau\left(Z_{n}\right)>\frac{-\log \left(2 \varepsilon / \delta_{0}\right)}{\vartheta_{c}}:=s$ if $Z_{n}$ is to intersect $N_{\varepsilon}(B)$ during a bound period. For cylinders with large return times, we can make a simple estimate using (14), 21) and Proposition 3.2 ,

$$
\begin{aligned}
\sum_{\substack{Z_{n} \in \mathfrak{c}_{\varepsilon} \varepsilon \\
\tau\left(Z_{n}\right)>s}} \sum_{\text {relevant } \ell} \tilde{\nu}_{t}\left(f_{\Delta}^{\ell} Z_{n}\right) & \leqslant \sum_{\substack{Z_{1} \in \mathcal{Z}_{1} \\
\tau\left(Z_{1}\right)>s}} \tau\left(Z_{1}\right) \tilde{\nu}_{t}\left(Z_{1}\right) \leqslant \sum_{\substack{Z_{1} \in Z_{1} \\
\tau\left(Z_{1}\right)>s}} \tau\left(Z_{1}\right) \lambda_{t}^{-\tau} e^{S_{\tau} \phi_{t}\left(Z_{1}\right)} \\
& \leqslant \sum_{\tau>s} \tau e^{\beta \tau} C_{0} e^{-\alpha \tau} \leqslant C^{\prime} s e^{(\beta-\alpha) s} \leqslant C^{\prime \prime} \varepsilon^{(\alpha-\beta) / \vartheta_{c}} \log \varepsilon
\end{aligned}
$$

where we have used the fact that $\lambda_{t}^{-1} \leqslant e^{\beta}$ and $\beta<\alpha$.

It remains to estimate the contribution from cylinders with $\tau<s$. Notice that by (23), all of these contributions are from pieces that are free at the time they intersect $N_{\varepsilon}(B)$. We fix $n \leq n_{0}$ and estimate the contributions from one $\mathfrak{C}_{\varepsilon, n}$ at a time. We also fix $t \in\left[t_{0}, 1\right]$. The argument for $t \in\left[1, t_{1}\right]$ is similar.

Notice that by definition of $\mathfrak{C}_{\varepsilon, n}$, if $Z_{n} \in \mathfrak{C}_{\varepsilon, n}$, then $\pi\left(f_{\Delta}^{\ell} Z_{n}\right) \cap N_{\varepsilon}(B) \neq 0$ for some (possibly more than one) $\ell<\tau\left(Z_{n}\right)$; but $\pi\left(f_{\Delta}^{\ell} Z_{n-1}\right) \not \subset N_{2 \varepsilon}(B)$ where $Z_{n-1}$ is the $(n-1)$-cylinder containing $Z_{n}$. This implies that $\left|\pi\left(f_{\Delta}^{\ell} Z_{n-1}\right)\right| \geq \varepsilon$. Then since $f_{\Delta}^{\tau^{n-1}\left(Z_{n}\right)}\left(Z_{n-1}\right)=\Delta_{0}$, we have by (A1),

$$
C_{0} \xi^{\tau^{n-1}\left(Z_{n}\right)-\ell}\left|\pi\left(f_{\Delta}^{\ell} Z_{n-1}\right)\right| \leqslant m_{1}\left(\Delta_{0}\right) \Longrightarrow \tau^{n-1}\left(Z_{n}\right)-\ell \leqslant \frac{-\log \left(C_{0} \varepsilon / \delta_{1}\right)}{\log \xi} .
$$


Since we are restricting to $\tau\left(Z_{n}\right)<s$, there are at most $s$ values of $\ell$ such that $\pi\left(f_{\Delta}^{\ell} Z_{n}\right) \cap N_{\varepsilon}(B) \neq \emptyset$, so

$$
\sum_{\substack{Z_{n} \in \mathfrak{C}_{\varepsilon, n}, n \\ \tau\left(Z_{n}\right)<s}} \sum_{\text {relevant } \ell} \tilde{\nu}_{t}\left(f_{\Delta}^{\ell} Z_{n}\right) \leqslant s \sum_{Z_{n} \in \mathfrak{C}_{\varepsilon, n}} \tilde{\nu}_{t}\left(Z_{n}\right)
$$

and since $s \approx \log \varepsilon$, it suffices to estimate the sum above.

Since $-S_{\tau^{n}} \varphi \leqslant \tau^{n} \chi_{M}$, we have,

$$
S_{\tau^{n}} \phi_{\Delta, t}=t S_{\tau^{n}} \varphi-\tau^{n} p_{t} \leqslant\left[(1-t) \chi_{M}-p_{t}\right] \tau^{n}+S_{\tau^{n}} \varphi .
$$

Setting $c_{t}=-\log \lambda_{t}+(1-t) \chi_{M}-p_{t}$ for brevity and using (25) together with (21) and (24), we obtain

$$
\begin{aligned}
& \sum_{Z_{n} \in \mathfrak{C}_{\varepsilon, n}} \tilde{\nu}_{t}\left(Z_{n}\right) \leqslant \sum_{Z_{n} \in \mathfrak{C}_{\varepsilon, n}} C \lambda_{t}^{-\tau^{n}\left(Z_{n}\right)} e^{S_{\tau^{n}\left(Z_{n}\right)} \phi_{\Delta, t}} \\
\leqslant & \sum_{Z_{n} \in \mathfrak{C}_{\varepsilon, n}} C \lambda_{t}^{-\tau^{n}\left(Z_{n}\right)} e^{\left[(1-t) \chi_{M}-p_{t}\right] \tau^{n}\left(Z_{n}\right)} m_{1}\left(Z_{n}\right) \\
\leqslant & C e^{c_{t}\left(\tau^{n-1}\left(Z_{n}\right)-\tau\left(Z_{n}\right)\right)} \sum_{Z_{n} \in \mathfrak{C}_{\varepsilon, n}} e^{c_{t}\left(\tau\left(Z_{n}\right)+\tau\left(F^{n-1} Z_{n}\right)\right)} m_{1}\left(Z_{n}\right) \\
\leqslant & C \varepsilon^{-c_{t} / \log \xi} \sum_{Z_{n} \in \mathfrak{C}_{\varepsilon, n}} e^{c_{t}\left(\tau\left(Z_{n}\right)+\tau\left(F^{n-1} Z_{n}\right)\right)} m_{1}\left(Z_{n}\right) .
\end{aligned}
$$

We split the sum up according to whether $\tau\left(Z_{n}\right)+\tau\left(F^{n-1} Z_{n}\right)$ is larger or smaller than $-\eta \log \varepsilon$ for some $\eta>0$ to be chosen later. Note that due to bounded distortion and the tail estimate, we have $m_{1}\left(x \in \Delta_{0}: \tau(x)+\tau\left(F^{n-1} x\right)=k\right) \leqslant C e^{-\alpha_{1} k}$. Thus for pieces with large return times, we have

$$
\begin{gathered}
\sum_{\tau+\tau \circ F^{n-1}>-\eta \log \varepsilon} e^{c_{t}\left(\tau\left(Z_{n}\right)+\tau\left(F^{n-1} Z_{n}\right)\right)} m_{1}\left(Z_{n}\right) \\
\leqslant C\left(e^{c_{t}-\alpha_{1}}\right)^{-\eta \log \varepsilon} \leqslant C \varepsilon^{\eta\left(\alpha_{1}-c_{t}\right)} .
\end{gathered}
$$

For pieces with small return times, we have

$$
\begin{gathered}
\sum_{\tau+\tau \circ F^{n-1}<-\eta \log \varepsilon} e^{c_{t}\left(\tau\left(Z_{n}\right)+\tau\left(F^{n-1} Z_{n}\right)\right)} m_{1}\left(Z_{n}\right) \\
\leqslant C\left(e^{c_{t}}\right)^{-\eta \log \varepsilon} \sum_{\substack{Z_{n} \\
\text { free at time } \ell \\
Z_{n} \in \mathfrak{C}_{\varepsilon, n}}} m_{1}\left(f_{\Delta}^{\ell} Z_{n}\right) \\
\leqslant C \varepsilon^{-\eta c_{t}} \varepsilon .
\end{gathered}
$$

where we have used the fact that the Lebesgue measure of free pieces that project into an interval of length $\varepsilon$ is bounded by const. $\varepsilon$ (see [BDM, Sect. 4.3, Step 1]).

In order to prove our estimate, we need the powers of $\varepsilon$ in both (27) and (28) to be positive after multiplying by the factor $\varepsilon^{-c_{t} / \log \xi}$ appearing in $(26)$. Thus we need,

$$
\frac{c_{t}}{\left(\alpha_{1}-c_{t}\right) \log \xi}<\eta<\frac{\log \xi-c_{t}}{c_{t} \log \xi}
$$

Such an $\eta$ always exists as long as

$$
c_{t}=-\log \lambda_{t}+(1-t) \chi_{M}-p_{t}<\frac{\alpha_{1} \log \xi}{\alpha_{1}+\log \xi},
$$

which holds for all small holes and for all $t$ close to 1 since $p_{1}=0$ and $\lambda_{t} \rightarrow 1$ as $H$ becomes small. 
We have estimated that the contribution to $\nu_{t}\left(N_{\varepsilon}(B)\right)$ from pieces in each $\mathfrak{C}_{\varepsilon, n}$ satisfies the desired bound. Since there are at most $n_{0}$ sets $\mathfrak{C}_{\varepsilon, n}$ in $\mathfrak{C}_{\varepsilon}$, and $n_{0} \leqslant-C \log \varepsilon$ by $(22)$, summing over $n$ adds only a logarithmic factor to our estimate, completing the proof that $\nu_{t} \in \mathcal{G}_{f}^{H}$.

7.2. Volume estimate. In this section, we will prove the following proposition, which then completes the proof of Theorem 3.4 .

Proposition 7.3. For each $t \in\left[t_{0}, t_{1}\right]$,

$$
-\mathfrak{e}\left(m_{t}\right) \geqslant \sup _{\mu \in \mathcal{G}_{f}^{H}}\left\{h(\mu)+t \int \varphi d \mu\right\}-P_{\mathcal{M}_{f}}(t \varphi) .
$$

We will estimate the $m_{t}$-mass of $I^{n}$ in terms of the pressure using the following partitions. Define $\mathcal{P}_{1}$ to be the partition of $I$ into open intervals whose endpoints are elements of Crit ${ }_{c}$ and let $\mathcal{P}_{n}:=\bigvee_{k=0}^{n-1} f^{-k} \mathcal{P}_{1}$. Similarly, let $\tilde{\mathcal{P}}_{1}$ denote the partition of $I$ induced by $\operatorname{Crit}_{c} \cup \partial H$ and define $\tilde{\mathcal{P}}_{n}$ analogously. We will estimate the mass of the elements of $\tilde{\mathcal{P}}_{n}$ in terms of Lyapunov exponents, and the number these cylinder sets in terms of the entropy. To get the estimate on the mass we construct another partition using the method of [Do, Section 4]. Note that this follows a very similar construction given in [L, Section 2]; see also [PU, Theorem 11.2.3] and [DWY, Section 3].

We define the natural extension as in $[\mathrm{L}]$. First define

$$
Y:=\left\{y=\left(y_{0}, y_{1}, \ldots\right): f\left(y_{i+1}\right)=y_{i} \in I\right\} .
$$

Define $\bar{f}^{-1}: Y \rightarrow Y$ by $\bar{f}^{-1}\left(\left(y_{0}, y_{1}, \ldots\right)\right)=\left(y_{1}, y_{2}, \ldots\right)$, so that $\bar{f}^{-1}$ is invertible with inverse $\bar{f}: \bar{f}^{-1} Y \rightarrow Y$ given by $\bar{f}\left(\left(y_{0}, y_{1}, \ldots\right)\right)=\left(f\left(y_{0}\right), y_{0}, y_{1}, \ldots\right)$. The projection $\Pi: Y \rightarrow I$ is defined as $\Pi: y=\left(y_{0}, y_{1}, \ldots\right) \mapsto y_{0}$. Hence $\Pi \circ \bar{f}=f \circ \Pi$. As in [Ro] (see also [PU, Section 2.7]), for any $\mu \in \mathcal{M}$ there is a unique $\bar{f}$-invariant probability measure $\bar{\mu}$ on $Y$ such that $\Pi_{*} \bar{\mu}=\mu$. Moreover, $\bar{\mu}$ is an ergodic invariant probability measure for $\bar{f}^{-1}$.

The triplet $(Y, \bar{f}, \bar{\mu})$ is called the natural extension of $(I, f, \mu)$. The following is a mild adaptation of [Do, Theorem 4.1], see also [L, Theorem 8].

Theorem 7.4. Suppose that $\mu \in \mathcal{G}_{f}^{H}$ has $\chi:=\int \log |D f| d \mu>0$ and let $(Y, \bar{f}, \bar{\mu})$ denote the natural extension of $(I, f, \mu)$. Then there exists a measurable function $g$ on $Y, 0<g<\frac{1}{2} \bar{\mu}$-a.e. such that for $\bar{\mu}$-a.e. $y \in Y$ there exists a set $V_{y} \subset Y$ with the following properties:

- $y \in V_{y}$ and $\Pi V_{y}=B(\Pi y, g(y))$;

- for each $n \in \mathbb{N}$, the set $\Pi \bar{f}^{-n} V_{y}$ is contained in $\tilde{\mathcal{P}}_{n}$;

- for all $y^{\prime} \in V_{y}$,

$$
\sum_{i=1}^{\infty}|\log | D f\left(\Pi \bar{f}^{-i} y^{\prime}\right)|-\log | D f\left(\Pi \bar{f}^{-i} y\right)||<\log 2
$$

- For each $\eta>0$ there exists a measurable function $\rho$ on $Y$ mapping into $[1, \infty)$ a.e. and such that

$$
\rho(y)^{-1} e^{n(\chi-\eta)}<\left|D f^{n}\left(\Pi \bar{f}^{-n} y\right)\right|<\rho(y) e^{n(\chi-\eta)},
$$

in particular, $\left|\Pi \bar{f}^{-n} V_{y}\right| \leqslant 2 \rho(y) e^{-n(\chi-\eta)}\left|\Pi V_{y}\right|$.

The only significant change to the proof given in [Do, Section 4] is to input information on the rate of approach of typical points to the boundary of the adapted partition $\tilde{\mathcal{P}}_{n}$, rather than simply $\mathcal{P}_{n}$, 
which is then applied in Lemma 4.5 of that paper. This information is contained in the following lemma.

Lemma 7.5. Given $\mu \in \mathcal{G}_{f}^{H}$, for each $\eta>0$, for $\bar{\mu}$-a.e. $y \in Y$ there exists $N \in \mathbb{N}$ such that $n \geqslant N$ implies $d\left(f^{k}\left(\Pi \bar{f}^{-n} y\right), \partial H\right)>e^{-\eta n}$ for all $0 \leqslant k \leqslant n-1$.

Proof. For any subset $A \subset I$ and $\delta>0$, set $\operatorname{dist}_{A, \delta}(x):=d(x, A)$ if $d(x, A)<\delta$ and 1 if $d(x, A) \geqslant \delta$. So if $\mu \in \mathcal{G}_{f}^{H}$ then $-\int \log \operatorname{dist}_{\partial H, \delta}(x) d \mu(x)<\infty$. Moreover, for any $\varepsilon>0$ there exists $\delta>0$ such that $-\int \log \operatorname{dist}_{\partial H, \delta}(x) d \mu(x)<\varepsilon$. Since $\left(Y, \bar{f}^{-1}, \bar{\mu}\right)$ is an ergodic dynamical system, by the ergodic theorem, for $\bar{\mu}$-a.e. $y \in Y$,

$$
\begin{aligned}
\frac{1}{n} \sum_{k=0}^{n-1} \log \operatorname{dist}_{\partial H, \delta}\left(f^{k}\left(\Pi \bar{f}^{-n} y\right)\right) & \rightarrow \int \log \operatorname{dist}_{\partial H, \delta}(\Pi y) d \bar{\mu}(y) \\
& =\int \log \operatorname{dist}_{\partial H, \delta}(x) d \mu(x) \text {, as } n \rightarrow \infty
\end{aligned}
$$

Now fix $\eta>0$ and choose $\delta$ so that $-\int \log \operatorname{dist}_{\partial H, \delta}(x) d \mu(x)<\eta / 2$. Then by the above limit, for $\bar{\mu}$-a.e. $y \in Y$, there exists $N=N(y)$ satisfying the statement of the lemma.

We need one more lemma before completing the proof of Proposition 7.3 .

Lemma 7.6. For each $t \in\left[t_{0}, t_{1}\right]$ and all $\delta>0$, there exists $\delta_{t}^{\prime}>0$ such that for every $x \in I$, $m_{t}((x-\delta, x+\delta))>\delta_{t}^{\prime}$.

Proof. This follows from the fact that $m_{t}$ gives open sets positive mass and the compactness of $I$.

Proof of Proposition 7.3. Fix $t \in\left[t_{0}, t_{1}\right]$. For any element $\tilde{\mathrm{C}}_{n} \in \tilde{P}_{n}$, by construction either $f^{n-1}\left(\tilde{\mathrm{C}}_{n}\right) \subset$ $H$ or $f^{n-1}\left(\tilde{\mathrm{C}}_{n}\right) \cap H=\emptyset$, so either $\tilde{\mathrm{C}}_{n}$ is contained in $\stackrel{\circ}{ }^{n-1}$ or it is outside $I^{n-1}$. Notice that the partition given by Theorem 7.4 is subordinate to $\tilde{P}_{1}$. This will give us subsets of cylinders $\tilde{\mathrm{C}}_{n} \in \tilde{\mathcal{P}}_{n}$ on which we have a good idea of the distortion.

Fix $\mu \in \mathcal{G}_{f}^{H}$ and the corresponding measure $\bar{\mu}$ in $Y$. Set $\eta>0$ and let $\rho$ be as in Theorem 7.4 For $\delta, K>0$, let $y \in \bar{I}_{\delta, K}:=\left\{y \in Y:\left|\Pi V_{y}\right|>\delta\right.$ and $\left.\rho(y)<K\right\}$. Fix $\varepsilon>0$ and choose $\delta>0$ small enough and $K$ large enough such that $\bar{\mu}\left(\bar{I}_{\delta, K}\right) \geqslant 1-\varepsilon$. By invariance, $\bar{\mu}\left(\bar{f}^{-n}\left(\bar{I}_{\delta, K}\right)\right)=$ $\mu\left(\Pi \bar{f}^{-n}\left(\bar{I}_{\delta, K}\right)\right) \geqslant 1-\varepsilon$.

Since $\mu$ is supported on $\stackrel{\circ}{I}^{\infty}$, for $\bar{\mu}$-a.e. $y \in \bar{I}_{\delta, K}$, this yields $x \in \stackrel{\circ}{I}^{\infty}$ such that $x=\Pi \bar{f}^{-n} y$. Moreover, defining

$$
I_{\delta, K, n}:=\left\{\Pi \bar{f}^{-n} y: y \in \bar{I}_{\delta, \kappa}\right\} \cap \stackrel{\circ}{I}^{\infty},
$$

we have $\mu\left(I_{\delta, K, n}\right) \geqslant 1-\varepsilon$ for all $n$.

For every $x \in I_{\delta, K, n}$ we take the corresponding $y \in \bar{I}_{\delta, K}$ and set $V_{x, n}:=\Pi \bar{f}^{-n} V_{y}$. By this setup, $\left|f^{n}\left(V_{x, n}\right)\right|>\delta$. By the Mean Value Theorem and the conformality of $m_{t}$ there exists $z \in \Pi \bar{f}^{-n} V_{y}$ such that

$$
m_{t}\left(V_{x, n}\right)=\left|D f^{n}(z)\right|^{-t} e^{-n p_{t}} m_{t}\left(f^{n}\left(V_{x, n}\right)\right) .
$$


Since $V_{x, n} \subset \tilde{\mathrm{C}}_{n}[x]$ and the two last parts of Theorem 7.4 we have

$$
\begin{aligned}
m_{t}\left(\tilde{\mathrm{C}}_{n}[x]\right) & \geqslant m_{t}\left(V_{x, n}\right) \geqslant \frac{1}{2^{t}}\left|D f^{n}(x)\right|^{-t} e^{-n p_{t}} m_{t}\left(f^{n}\left(V_{x, n}\right)\right) \\
& \geqslant \frac{1}{2^{t} K} e^{-n t(\chi(\mu)+\eta)} e^{-n p_{t}} m_{t}\left(f^{n}\left(V_{x, n}\right)\right) \geqslant \frac{1}{2^{t} K} e^{-n t(\chi(\mu)+\eta)} e^{-n p_{t}} \delta_{t}^{\prime},
\end{aligned}
$$

for $\delta_{t}^{\prime}>0$ depending on $\delta>0$ as in Lemma 7.6.

Now we use the Shannon-McMillan-Breiman Theorem, see for example [PU, Section 2.5], to assert that on a set $E \subset I^{\infty}$ of $\mu$-measure at least $1-\varepsilon, \mu\left(\tilde{\mathrm{C}}_{n}[x]\right) \leqslant e^{-n(h(\mu)-\eta)}$ for $x \in E$ and $n$ sufficiently large. Thus the number of distinct cylinders $\tilde{\mathrm{C}}_{n}[x]$ with $x \in E \cap I_{\delta, K, n}$ is at least $(1-2 \varepsilon) e^{n(h(\mu)-\eta)}$ for all $n$ large enough. Notice that such cylinders are in $I^{n-1}$ by definition of $\tilde{\mathcal{P}}_{n}$. Combining this with 29 , we obtain,

$$
m_{t}\left(\stackrel{\circ}{I}^{n-1}\right) \geqslant \sum_{x \in I_{\kappa, \delta, n} \cap E} m_{t}\left(\tilde{\mathrm{C}}_{n}[x]\right) \geqslant(1-2 \varepsilon) e^{n(h(\mu)-\eta)} \frac{\delta_{t}^{\prime}}{2^{t} K} e^{-n t(\chi+\eta)} e^{-n p_{t}} .
$$

So by the arbitrary choice of $\eta>0$, taking logs of both sides, dividing by $n$ and letting $n \rightarrow \infty$ yields $m_{t}\left(I^{n-1}\right) \geqslant h(\mu)-t \chi(\mu)$. Taking a supremum over all $\mu \in \mathcal{G}_{f}^{H}$, the proposition is proved.

\section{A Bowen Formula: Proof of Theorem 3.5}

Theorem 8.1. Under the assumptions of Theorem 3.4. $\operatorname{dim}_{H}\left(I^{\infty}\right)=t^{*}$ where $t^{*}$ is the unique value of $t$ such that $P_{\Delta}\left(t \varphi^{H}\right)=0$.

Proof. We will use the main result of [I], which is a Bowen formula for countable Markov shifts, but similarly to applications in that paper, extends to our case here as follows. As in Theorem 3.1(b), for $h$ small enough and $H \in \mathcal{H}(h), \operatorname{dim}_{H}\left(\stackrel{\circ}{I}^{\infty}\right)=\operatorname{dim}_{H}\left(\stackrel{\circ}{\Delta}^{\infty}\right)$. The structure of $\Delta$ allows us to code it as a countable Markov shift. The required result then follows as a consequence of [I], where the corresponding 'metric potential' as in [I, (3)] is $t \varphi_{\Delta}$ using the Hölder regularity provided by Lemma 4.1 .

Now [I, Theorem 3.1] gives $\operatorname{dim}_{H}\left(\AA^{\infty}\right)=t^{*}$ where $t^{*}$ is the unique value of $t$ satisfying $P_{\mathcal{M}_{f_{\Delta}}^{H}}\left(t^{*} \varphi_{\Delta}\right)=$ 0 . Then following the proof of Theorem 3.4 from Section 7 yields $P_{\Delta}\left(t^{*} \varphi^{H}\right)=0$.

Recall that the invariant measure $\nu_{t}^{H}$ constructed in Section 7 achieves the supremum in the variational formula and belongs to $\mathcal{G}_{f}^{H}$. Also, $\nu_{f}^{H}$ lifts to $\Delta$, so that it is included in the pressure $P_{\Delta}\left(t \varphi^{H}\right)$. Thus $P_{\mathcal{G}_{f}^{H}}\left(t^{*} \varphi^{H}\right)=0$, completing the proof of Theorem 3.5

\section{Zero-Hole Limit: Proofs of Theorems 3.6 and 3.7}

Recall that for Theorem 3.6, we consider holes of the form $H_{\varepsilon}=(z-\varepsilon, z+\varepsilon)$, for $z \in I$ satisfying the following condition:

There exist $\varsigma, \delta_{z}>0$ with $\varsigma<\min \left\{2 \vartheta_{c}, \alpha / s_{t}\right\}$, such that $\left|f^{n}(c)-z\right| \geqslant \delta_{z} e^{-n \varsigma}$ for all $n \geqslant 0$.

Here $\vartheta_{c}$ is from $(\mathrm{C} 2), \alpha$ is from Proposition 3.2 and $s_{t}$ is the local dimension of $m_{t}$ at $z$ given in Lemma 9.5 below. The fact that this is a generic condition with respect to both $m_{t}$ and $\mu_{t}$ is proved in Lemma 9.3. Recall that (31) is part of condition (P). 
Because we will need to maintain careful control of the constants involved in the tower construction along our sequence of holes, we recall the following set of choices explicitly.

Fix $z \in I$ satisfying (31) so that Crit hole $=\{z\}$ satisfies (H2) and (2) with $\delta=\delta_{0}$. This fixes $n\left(\delta_{0}\right)$ and all appearances of $\delta$ in $(\mathrm{C} 1)-(\mathrm{C} 2)$ to have value $\delta_{0}$. As before, denote by $\mathcal{H}(h)$ the family of intervals $H$ such that $z \in H, m_{1}(H) \leqslant h$ and $H$ satisfies (H1). All our intervals $H_{\varepsilon}$ are required to belong to $\mathcal{H}(h)$ for some $h>0$.

9.1. Preparatory Lemmas and the proof of Theorem 3.6. We adopt the following notation for our family of inducing schemes: $\left(\cup_{i} X_{\varepsilon}^{i}, F_{\varepsilon}, \tau_{\varepsilon}, H_{\varepsilon}\right)$, so that $F\left(X_{\varepsilon}^{i}\right)=X$. Note that in this notation, $X$ is fixed for all $H_{\varepsilon} \in \mathcal{H}(h)$.

When we view $H_{\varepsilon}$ as a hole for the open system, if $k<\tau\left(X_{\varepsilon}^{i}\right)$ is the first time that $f^{k}\left(X_{\varepsilon}^{i}\right) \subset H$, we define $\stackrel{\circ}{\tau}_{\varepsilon}\left(X_{\varepsilon}^{i}\right)=k$; otherwise if $X_{\varepsilon}^{i}$ returns to $X$ before encountering $H$, we set $\stackrel{\circ}{\tau}_{\varepsilon}\left(X_{\varepsilon}^{i}\right)=\tau_{\varepsilon}\left(X_{\varepsilon}^{i}\right)$. The induced map for the open system $\stackrel{\circ}{F}_{\varepsilon}$ is defined similarly so that $\stackrel{\circ}{F}_{\varepsilon}(x)=F_{\varepsilon}(x)$ whenever $x \in X$ returns to $X$ without entering $H$ along the way.

Now for $H \in \mathcal{H}(h)$, we have a tower $\left(f_{\Delta}, \Delta(H)\right)$ constructed so that $\pi^{-1} H$ is a union of 1-cylinders $\tilde{H}_{\ell, j}$. We recall that the family of towers corresponding to $\mathcal{H}(h)$ satisfy (P1)-(P4) with uniform constants. For our function space $\mathcal{B}$ on the tower, we choose $\beta<\min \left\{\alpha, t_{0} \log \xi\right\}$ as in Section 6.1 and add the further requirement that $\beta<\alpha-\varsigma s_{t}$, where $\varsigma$ is from (31) and $s_{t}$ is the scaling exponent from Lemma 9.5. Note that with this choice of $\beta$, the exponent $\varsigma$ necessarily satisfies

$$
\varsigma<\min \left\{(\alpha-\beta) / s_{t}, 2 \vartheta_{c}\right\} .
$$

Also, if we need to shrink $\beta$ in what follows, this does not affect the value of $\varsigma$, which is fixed and depends on $z$.

Let $g_{t}^{H} \in \mathcal{B}$ denote the eigenfunction corresponding to $\lambda_{t}^{H}$ for $\mathcal{L}_{\phi_{t}^{H}}:=\stackrel{\circ}{\mathcal{L}}_{\phi_{\Delta, t}}$. We have introduced this new notation for the transfer operator with the hole in order to make dependence on $H$ explicit. We drop the subscript $\Delta$ since all the objects we work with in this section will be on the tower. We denote by $g_{t}^{0} \in \mathcal{B}$ the invariant probability density for the transfer operator without the hole, $\mathcal{L}_{\phi_{t}}$. We remark that $g_{t}^{0}$ also depends on $H$ since different $H$ induce different towers $\Delta(H)$, but the projection $\mathcal{P}_{\pi} g_{t}^{0}$ is independent of $H$.

We use the notation $\AA^{n}(H)$ to indicate the set of points that has not escaped $\Delta(H)$ by time $n$ and set $\AA^{0}(H)=\AA(H)$. Recall the notation $\tilde{H}=\pi^{-1} H, \tilde{H}=\cup_{\ell} \tilde{H}_{\ell}$ and $\tilde{H}_{\ell}=\cup_{j} \tilde{H}_{\ell, j}$.

With our definitions, $g_{t}^{H} \equiv 0$ on $\tilde{H}$ and all columns above each $\tilde{H}_{\ell, j}$. Since it will be convenient to make our estimates directly on $\tilde{H}$, we extend $g_{t}^{H}$ to $\tilde{H}$ by $g_{t}^{H}(x)=\left(\lambda_{t}^{H}\right)^{-1} g\left(f_{\Delta}^{-1} x\right)$ for $x \in \tilde{H}$. Note that this extended version of $g_{t}^{H}$ is still 0 in the column above each $\tilde{H}_{\ell, j}$. We normalize $g_{t}^{H}$ so that, $\int_{\Delta(H)} g_{t}^{H} d \bar{m}_{t}=1$.

If we redefine $\mathcal{L}_{\phi_{t}^{H}} \psi:=\mathcal{L}_{\phi_{t}}\left(1_{\triangle(H)} \psi\right)$ (rather than $\mathcal{L}_{\phi_{t}}\left(1_{\Delta^{1}(H)} \psi\right)$ ), it extends the operator so that $\mathcal{L}_{\phi_{t}^{H}} g_{t}^{H}(x)=\lambda_{t}^{H} g_{t}^{H}(x)$ for $x \in \tilde{H}$. Note, however, that $\mathcal{L}_{\phi_{t}^{H}}$ still does not let mass map out of $\tilde{H}$ so that no mass maps to the columns above $\tilde{H}$. We will use this extended definition of $\mathcal{L}_{\phi_{t}^{H}}$ for the remainder of this section.

Since $\mathfrak{e}\left(m_{t}, H_{\varepsilon}\right)=-\log \lambda_{t}^{H_{\varepsilon}}$, proving Theorem 3.6 is equivalent to estimating

$$
\lim _{\varepsilon \rightarrow 0} \frac{1-\lambda_{t}^{H_{\varepsilon}}}{\mu_{t}\left(H_{\varepsilon}\right)},
$$


which we now start to do.

We begin with the key observation that by definition of $\mathcal{L}_{\phi_{t}^{H}}$ and $g_{t}^{H}$,

$$
\lambda_{t}^{H}=\lambda_{t}^{H} \int_{\Delta(H)} g_{t}^{H} d \bar{m}_{t}=\int_{\Delta(H)} \mathcal{L}_{\phi_{t}^{H}} g_{t}^{H} d \bar{m}_{t}=\int_{\AA^{0}(H)} g_{t}^{H} d \bar{m}_{t}
$$

So now for any $n \geqslant 0$, using the conditional invariance of $g_{t}^{H}$, we write

$$
\begin{aligned}
1-\lambda_{t}^{H} & =\int_{\Delta(H)} g_{t}^{H} d \bar{m}_{t}-\int_{\dot{\Delta}^{0}(H)} g_{t}^{H} d \bar{m}_{t}=\int_{\tilde{H}} g_{t}^{H} d \bar{m}_{t} \\
& =\left(\lambda_{t}^{H}\right)^{-n} \int_{\tilde{H}}\left(\mathcal{L}_{\phi_{t}^{H}}^{n} g_{t}^{H}-\mathcal{L}_{\phi_{t}}^{n} g_{t}^{0}\right) d \bar{m}_{t}+\left(\lambda_{t}^{H}\right)^{-n} \int_{\tilde{H}} g_{t}^{0} d \bar{m}_{t} .
\end{aligned}
$$

The following lemmas, the proofs of which we give later, will allow us to prove Theorem 3.6 .

Lemma 9.1. The transfer operators $\mathcal{L}_{\phi_{t}}$ and $\mathcal{L}_{\phi_{t}^{H}}$ have a uniform spectral gap for all $H \in \mathcal{H}(h)$ with $h$ sufficiently small. More precisely, there exist $C_{2}>0$ and $\sigma_{0}<1$ such that for all $\psi \in \mathcal{B}$ and $n \geqslant 0$,

$$
\begin{aligned}
\left\|\mathcal{L}_{\phi_{t}}^{n} \psi-c_{0}(\psi) g_{t}^{0}\right\|_{\mathcal{B}} & \leqslant C_{2} \sigma_{0}^{n}\|\psi\|_{\mathcal{B}}\left\|g_{t}^{0}\right\|_{\mathcal{B}} \quad \text { and } \\
\left\|\left(\lambda_{t}^{H}\right)^{-n} \mathcal{L}_{\phi_{t}^{H}}^{n} \psi-c_{H}(\psi) g_{t}^{H}\right\|_{\mathcal{B}} & \leqslant C_{2} \sigma_{0}^{n}\|\psi\|_{\mathcal{B}}\left\|g_{t}^{H}\right\|_{\mathcal{B}}
\end{aligned}
$$

where the constants $c_{H}(\psi)$ and $c_{0}(\psi)$ represent the magnitude of the projections of $\psi$ onto the eigenspaces spanned by $g_{t}^{H}$ and $g_{t}^{0}$, respectively. Moreover, $c_{H}(\psi) \rightarrow c_{0}(\psi)$ as $\operatorname{diam}(H) \rightarrow 0$ in $\mathcal{H}(h)$.

Lemma 9.2. There exists $C_{3}>0$ such that if $z$ satisfies (31) then for $h$ sufficiently small and for each $H \in \mathcal{H}(h)$,

$$
\sum_{\ell \geqslant 0} e^{\beta \ell} \bar{m}_{t}\left(\tilde{H}_{\ell}\right) \leqslant C_{3} \mu_{t}(H)
$$

Lemma 9.3. Condition (31) is generic with respect to both $\mu_{t}$ and $m_{t}$.

The three lemmas above are sufficient for the proof of the generic case. The next lemma is required in the periodic case.

Lemma 9.4. Suppose that $z$ is a periodic point of period $p$ and $\frac{d \mu_{t}}{d m_{t}}(z) \in(0, \infty)$. Given $n \in \mathbb{N}$ we can choose $h$ so small that if $H \in \mathcal{H}(h)$ then

$$
\mu_{t}(H) e^{S_{p} \phi_{t}(z)}(1-\gamma(n)) \leqslant \int_{\tilde{H}}\left(\mathcal{L}_{\phi_{t}}^{n}-\mathcal{L}_{\phi_{t}^{H}}^{n}\right) g_{0} d \bar{m}_{t} \leqslant \mu_{t}(H) e^{S_{p} \phi_{t}(z)}(1+\gamma(n))
$$

where $\gamma(n) \rightarrow 0$ as $n \rightarrow \infty$ independently of $H \in \mathcal{H}(h)$.

Proof of Theorem 3.6. Non-periodic case. Fix $\varepsilon>0$ and choose $h>0$ so small that $\lambda_{t}^{H} \geqslant \sigma_{0}^{1 / 2}$ for all $H \in \mathcal{H}(h)$. Next choose $n$ so large that $\sigma_{0}^{n / 2}<\varepsilon$. Finally, choose $H$ sufficiently small that $\left(\lambda_{t}^{H}\right)^{-n} \leqslant 1+\varepsilon$ and $f_{\Delta}^{-k} \tilde{H} \cap \tilde{H}=\emptyset$ for all $1 \leqslant k \leqslant n$. This last choice is possible using the aperiodicity of $z$. 
Note that due to this last choice, we have $\mathcal{L}_{\phi_{t}^{H}}^{n}=\mathcal{L}_{\phi_{t}}^{n}$ when integrated over $\tilde{H}$. Now the first term on the right hand side of $(33)$ becomes

$$
\begin{array}{r}
\left(\lambda_{t}^{H}\right)^{-n} \int_{\tilde{H}} \mathcal{L}_{\phi_{t}}^{n}\left(g_{t}^{H}-g_{t}^{0}\right) d \bar{m}_{t} \leqslant\left(\lambda_{t}^{H}\right)^{-n} \sum_{\ell \geqslant 1} e^{\beta \ell}\left\|\mathcal{L}_{\phi_{t}}^{n} g_{t}^{H}-g_{t}^{0}\right\|_{\mathcal{B}} \bar{m}_{t}\left(\tilde{H}_{\ell}\right) \\
\leqslant C_{2} \sigma_{0}^{n / 2}\left\|g_{t}^{H}\right\|_{\mathcal{B}}\left\|g_{t}^{0}\right\|_{\mathcal{B}} \sum_{\ell \geqslant 1} e^{\beta \ell} \bar{m}_{t}\left(\tilde{H}_{\ell}\right) \leqslant C_{2} \varepsilon\left\|g_{t}^{H}\right\|_{\mathcal{B}}\left\|_{g_{t}^{0}}\right\|_{\mathcal{B}} \mu_{t}(H),
\end{array}
$$

where we have used Lemmas 9.1 9.3 as well as the fact that $g_{t}^{0}$ is uniformly bounded above and below on $\Delta$ (see [BDM, Prop. 2.4]). Note that in the application of Lemma 9.3, we use the fact that $c_{0}\left(g_{t}^{H}\right)=\int_{\Delta(H)} g_{t}^{H} d m_{t}=1$. Now $\left\|g_{t}^{H}\right\|_{\mathcal{B}}$ and $\left\|g_{t}^{0}\right\|_{\mathcal{B}}$ are uniformly bounded for all $H \in \mathcal{H}(h)$ with constants depending only on (P1)-(P4) [BDM, Prop. 2.3]. Thus the first term of (33) can be made an arbitrarily small multiple of $\mu_{t}(H)$.

The second term of $(33)$ is simply bounded by

$$
\int_{\tilde{H}} g_{t}^{0} d \bar{m}_{t} \leqslant\left(\lambda_{t}^{H}\right)^{-n} \int_{\tilde{H}} g_{t}^{0} d \bar{m}_{t} \leqslant(1+\varepsilon) \int_{\tilde{H}} g_{t}^{0} d \bar{m}_{t} .
$$

Since $\int_{\tilde{H}} g_{t}^{0} d \bar{m}_{t}=\bar{\mu}_{t}(\tilde{H})=\mu_{t}(H)$, we have shown that

$$
\frac{1-\lambda_{t}^{H}}{\mu_{t}(H)}=1+\epsilon(H)
$$

where $\epsilon(H) \rightarrow 0$ as $m_{t}(H) \rightarrow 0$.

Periodic case. We split the first term on the right side of (33) into two and renormalize $g_{t}^{0}$ by $c_{H}\left(g_{t}^{0}\right)$ :

$$
\begin{aligned}
1-\lambda_{t}^{H}= & \left(\lambda_{t}^{H}\right)^{-n} c_{H}\left(g_{t}^{0}\right)^{-1} \int_{\tilde{H}} \mathcal{L}_{\phi_{t}^{H}}^{n}\left(c_{H}\left(g_{t}^{0}\right) g_{t}^{H}-g_{t}^{0}\right) d \bar{m}_{t} \\
& +\left(\lambda_{t}^{H}\right)^{-n} c_{H}\left(g_{t}^{0}\right)^{-1} \int_{\tilde{H}}\left(\mathcal{L}_{\phi_{t}^{H}}^{n}-\mathcal{L}_{\phi_{t}}^{n}\right) g_{t}^{0} d \bar{m}_{t}+\left(\lambda_{t}^{H}\right)^{-n} c_{H}\left(g_{t}^{0}\right)^{-1} \int_{\tilde{H}} g_{t}^{0} d \bar{m}_{t} .
\end{aligned}
$$

As before, we fix $\varepsilon>0$ and choose $n$ so large that $\sigma_{0}^{n / 2}<\varepsilon$ and $\gamma(n)$ from Lemma 9.4 is less than $\varepsilon$. Next choose $h>0$ so small that $\lambda_{t}^{H} \geqslant \sigma_{0}^{1 / 2}$ and $\left(\lambda_{t}^{H}\right)^{-n} \leqslant 1+\varepsilon$ for all $H \in \mathcal{H}(h)$. Finally, since $c_{0}\left(g_{t}^{0}\right)=1$, by Lemma 9.1. we may shrink $h$ further so that $c_{H}\left(g_{t}^{0}\right) \in(1-\varepsilon, 1+\varepsilon)$ and for our given $n$, the holes are small enough that the conclusion of Lemma 9.4 holds.

Using Lemmas 9.1 and 9.2 again, the first term in 35 is bounded by

$$
\left|\left(\lambda_{t}^{H}\right)^{-n} c_{H}\left(g_{t}^{0}\right)^{-1} \int_{\tilde{H}} \mathcal{L}_{\phi_{t}^{H}}^{n}\left(c_{H}\left(g_{t}^{0}\right) g_{t}^{H}-g_{t}^{0}\right) d \bar{m}_{t}\right| \leqslant C \varepsilon\left\|g_{t}^{H}\right\|_{\mathcal{B}}\left\|_{t}^{0}\right\|_{\mathcal{B}} \mu_{t}(H) .
$$

Using Lemma 9.4, the second term in (35) is bounded above and below by

$$
-\mu_{t}(H) e^{S_{p} \phi_{t}(z)} \frac{(1+\varepsilon)^{2}}{1-\varepsilon} \leqslant \frac{\left(\lambda_{t}^{H}\right)^{-n}}{c_{H}\left(g_{t}^{0}\right)} \int_{\tilde{H}}\left(\mathcal{L}_{\phi_{t}^{H}}^{n}-\mathcal{L}_{\phi_{t}}^{n}\right) g_{t}^{0} d \bar{m}_{t} \leqslant-\mu_{t}(H) e^{S_{p} \phi_{t}(z)} \frac{1-\varepsilon}{1+\varepsilon} .
$$

Finally, the third term of (35) is bounded above and below as in (34).

Putting these three estimates together, we conclude,

$$
1-e^{S_{p} \phi_{t}(z)}-C \varepsilon \leqslant \frac{1-\lambda_{t}^{H}}{\mu_{t}(H)} \leqslant 1-e^{S_{p} \phi_{t}(z)}+C \varepsilon,
$$

for a constant $C$ independent of $H$. Since $\varepsilon$ is arbitrary, this completes the proof of the theorem. 
The proof of Theorem 3.7 requires some minor adaptations of our preparatory lemmas, so we will leave its proof until we have proved those lemmas.

\subsection{Proofs of generic lemmas.}

Proof of Lemma 9.1. The proof follows from the fact that for the transfer operator without the hole $\mathcal{L}_{\phi_{t}}, e^{-\beta}$ gives an upper bound on the second largest eigenvalue as well as a bound on the essential spectral radius. This is proved in [M, Theorem 1.4 and Section 4.1]. There it is shown that in our setup ( $\operatorname{since} \beta$ is very close to 0 ), a constructive bound on the second largest eigenvalue is given by $\tanh (\mathcal{R} / 2)$ where $\mathcal{R}=\log \frac{1+e^{-\beta}}{1-e^{-\beta}}$. Simplifying this expression yields $\tanh (\mathcal{R} / 2)=e^{-\beta}$.

By our Proposition 3.2, we have uniform control of the tails of the return time $\tau$ for all $H \in \mathcal{H}(h)$ and $t \in\left[t_{0}, t_{1}\right]$. Thus we work with a fixed $\beta>0$ (chosen in Section 9.1) in all our towers which gives a uniform bound on the second largest eigenvalue of $\mathcal{L}_{\phi_{t}}$.

The fact that the spectrum and spectral projectors of $\mathcal{L}_{\phi_{t}^{H}}$ are close to that of $\mathcal{L}_{\phi_{t}}$ outside the disk of radius $e^{-\beta}$ follows from [DW, Lemma 3.6] (which in turn is an application of [KL1] adapted to sequences of Young tower constructions). There, it is shown that the eigenvalues of $\mathcal{L}_{\phi_{t}^{H}}$ and $\mathcal{L}_{\phi_{t}}$ outside the disk of radius $e^{-\beta}$ vary by at most $\mathcal{O}\left(h^{\epsilon}\right)$ for all $H \in \mathcal{H}(h)$ and some $\epsilon>0$.

Choosing $h$ sufficiently small, we may guarantee that $\lambda_{t}^{H} \geqslant e^{-\beta / 3}$ and the second largest eigenvalue of $\mathcal{L}_{\phi_{t}^{H}}$ is at most $e^{-2 \beta / 3}$. This ensures that $\sigma_{0}<1$ in the statement of the lemma is at most $e^{-\beta / 3}$ for all $H \in \mathcal{H}(h)$.

In addition, letting $\Pi_{\lambda^{H}}$ and $\Pi_{1}$ denote the spectral projections onto the eigenspaces associated with $g_{t}^{H}$ and $g_{t}^{0}$, respectively, the same perturbative results from [DW] used above imply that for $\psi \in \mathcal{B}$,

$$
\begin{aligned}
\left|c_{H}(\psi)-c_{0}(\psi)\right| & =\left|\int_{\Delta(H)} \Pi_{\lambda^{H}} \psi-\Pi_{1} \psi d m_{t}\right| \\
& \leqslant\left\|\Pi_{\lambda^{H}} \psi-\Pi_{1} \psi\right\|_{\mathcal{B}} \sum_{\ell} m_{t}\left(\Delta_{\ell}\right) e^{\beta \ell} \leqslant C h^{\epsilon},
\end{aligned}
$$

where $C$ is independent of $H \in \mathcal{H}(h)$, proving the continuity of $c_{H}(\psi)$ in $H$.

Before giving the proof of Lemma 9.2, we need the following important fact about the scaling of the measure $m_{t}$ on small sets.

Lemma 9.5. Suppose that $z$ is either a $\mu_{t}$-typical point or a periodic point with $\frac{d \mu_{t}}{d m_{t}}(z) \in(0, \infty)$ and set $s_{t}:=t+\frac{p_{t}}{\lambda(z)}$. Then for each $\varepsilon>0$, there exists $\delta(\varepsilon)>0$ such that for all $\delta \in(0, \delta(\varepsilon))$,

$$
(2 \delta)^{s_{t}+\varepsilon} \leqslant m_{t}\left(B_{\delta}(z)\right) \leqslant(2 \delta)^{s_{t}-\varepsilon} .
$$

Moreover, $s_{t}>0$, and in the case that $z$ is $\mu_{t}$-typical then $s_{t}$ can also be written as $\frac{h\left(\mu_{t}\right)}{\lambda\left(\mu_{t}\right)}$, which is $<1$ whenever $t \neq 1$.

Proof. In the case that $z$ is $\mu_{t}$-typical, (36) follows immediately from the definition of local dimension $d_{\mu_{t}}$ at $x$, where

$$
d_{\mu_{t}}(x)=\lim _{\delta \rightarrow 0} \frac{\log \mu_{t}\left(B_{\delta}(x)\right)}{\log 2 \delta}
$$


whenever the limit exists. For $\mu_{t}$-typical points this is always equal to the dimension of the measure $\frac{h\left(\mu_{t}\right)}{\lambda\left(\mu_{t}\right)}$, see [Ho]. So $s_{t}=\frac{h\left(\mu_{t}\right)}{\lambda\left(\mu_{t}\right)}=t+\frac{p_{t}}{\lambda\left(\mu_{t}\right)}=t+\frac{p_{t}}{\lambda(z)}$. By the Ruelle-Pesin formula, as well as the obvious fact that the dimension of a measure is bounded by the dimension of the space, $s_{t} \leqslant 1$. Moreover, since the unique invariant measure which has dimension 1 is $\mu_{1}$, we have $s_{t}<1$ whenever $t \neq 1$, see for example $[\mathrm{L}]$. We can make the switch from the invariant measure $\mu_{t}$ to the conformal measure $m_{t}$ using the fact that the density at typical $z$ exists and takes a value in $(0, \infty)$.

In the case when $f^{q}(z)=z$, first notice that elementary arguments on the pressure function imply that for any $t$ where an equilibrium state of positive entropy $\mu_{t}$ exists, $p_{t}>-t \lambda(z)$ for any periodic point $z$. Therefore, $s_{t}$ in this case is strictly positive. Moreover, $S_{q} \phi(z)<0$.

For the scaling properties of $m_{t}$ around $z$, the situation is in many ways simpler than the typical case, although we can't call on the powerful theory of local dimension described above. The proof is similar to [FFT, Lemma 4.1] so we only sketch it. We use the fact that we can pick $\hat{\delta}>0$ such that on $B_{\hat{\delta}}(z),\left|D f^{q}\right| \sim\left|D f^{q}(z)\right|$, where $\sim$ denotes a uniform constant depending only on $\hat{\delta}$ and the distortion constant $C_{d}$ from (A1). Then for a ball of size $\delta \in(0, \hat{\delta})$, conformality implies that for $n=\left\lfloor\frac{\log (\hat{\delta} / \delta)}{\log \left|D f^{q}(z)\right|}\right\rfloor$,

$$
m_{t}\left(B_{\delta}(z)\right) \sim\left|D f^{n q}(z)\right|^{-t} e^{-n q p_{t}} m_{t}\left(B_{\hat{\delta}}(z)\right) \sim \delta^{t} e^{-n q p_{t}}
$$

Hence

$$
\lim _{\delta \rightarrow 0} \frac{\log m_{t}\left(B_{\delta}(x)\right)}{\log 2 \delta}=t+\frac{q p_{t}}{\log \left|D f^{q}(z)\right|}=t+\frac{p_{t}}{\lambda(z)}
$$

as required.

Proof of Lemma 9.2. Holes in $\Delta$ are created in one of two ways: When $f^{n} X$ encounters $H$ during a bound period or when it is free. We split the relevant sum into these pieces,

$$
\sum_{\ell} \bar{m}_{t}\left(H_{\ell}\right) e^{\beta \ell}=\sum_{\text {bound }} \bar{m}_{t}\left(H_{\ell}\right) e^{\beta \ell}+\sum_{\text {free }} \bar{m}_{t}\left(H_{\ell}\right) e^{\beta \ell}
$$

Estimate on bound pieces. Since $\bar{m}_{t}\left(H_{\ell}\right)=\bar{m}_{t}\left(f_{\Delta}^{-\ell} H_{\ell}\right)$, we will estimate the sum over all 1-cylinders $X_{\varepsilon}^{i}$ such that $f^{\ell}\left(X_{\varepsilon}^{i}\right) \subset H$ and $X_{\varepsilon}^{i}$ is bound at time $\ell, \ell<\tau\left(X_{\varepsilon}^{i}\right)$.

If $X_{\varepsilon}^{i}$ is bound at time $\ell$, then $\left|f^{\ell}(x)-f^{\ell}(c)\right| \leqslant \delta_{0} e^{-2 \vartheta_{c} \ell}$, for each $x \in X_{\varepsilon}^{i}$. Thus fixing $x \in X_{\varepsilon}^{i}$ and using (31), we obtain

$$
\delta_{z} e^{-\varsigma \ell} \leqslant\left|f^{n}(c)-z\right| \leqslant\left|f^{n}(c)-f^{n}(x)\right|+\left|f^{n}(x)-z\right| \leqslant \delta_{0} e^{-2 \vartheta_{c} \ell}+|H| / 2,
$$

Since $\varsigma<2 \vartheta_{c}$ by (32), this inequality can only be satisfied by sufficiently large $\ell$ and if $\delta_{z}<\delta_{0}$, by finitely many small values of $\ell$ as well. So we assume the worst case scenario, that $\delta_{z}<\delta_{0}$. The finitely many $\ell$ must satisfy $\ell \leqslant \frac{\log \left(\delta_{0} / \delta_{z}\right)}{2 \vartheta_{c}-\varsigma}$. On the other hand, the sufficiently large $\ell$ must satisfy,

$$
\delta_{z} e^{-\varsigma \ell} \leqslant|H| / 2 \Longrightarrow \ell \geqslant \frac{-\log \left(|H| / 2 \delta_{z}\right)}{\varsigma}
$$


Putting these estimates together, we have the following estimate on the contribution from bound pieces,

$$
\begin{aligned}
\sum_{\text {bound }} \bar{m}_{t}\left(H_{\ell}\right) e^{\beta \ell} & \leqslant \sum_{\ell \leqslant \frac{\log \left(\delta_{0} / \delta_{z}\right)}{2 \vartheta_{c}-\varsigma}} \bar{m}_{t}\left(H_{\ell}\right) e^{\beta \ell}+\sum_{\ell \geqslant \frac{-\log \left(|H| / 2 \delta_{z}\right)}{\varsigma}} \bar{m}_{t}\left(H_{\ell}\right) e^{\beta \ell} \\
& \leqslant C e^{\beta \frac{\log \left(\delta_{0} / \delta_{z}\right)}{2 \vartheta_{c}-\varsigma}} \sum_{\ell} \bar{\mu}_{t}\left(H_{\ell}\right)+\sum_{\ell \geqslant \frac{-\log \left(|H| / 2 \delta_{z}\right)}{\varsigma}} C e^{-(\alpha-\beta) \ell} \\
& \leqslant C e^{\beta \frac{\log \left(\delta_{0} / \delta_{z}\right)}{2 \vartheta_{c}-\varsigma}} \mu_{t}(H)+C \delta_{z}^{(\beta-\alpha) / \varsigma}|H|^{(\alpha-\beta) / \varsigma},
\end{aligned}
$$

where we have used the fact that $\bar{\mu}_{t}$ has density with respect to $\bar{m}_{t}$ uniformly bounded above and below on $\Delta$ in the last line. By $(32)$, the exponent of $|H|$ in the last term is greater than $s_{t}=t+\frac{p_{t}}{\lambda(z)}$ from Lemma 9.5. So remembering that $H_{\varepsilon}=B_{\varepsilon}(z)$ and by choosing $h$ sufficiently small, we have $|H|^{(\alpha-\beta) / \varsigma} \leqslant m_{t}(H) \leqslant C \mu_{t}(H)$, where we have used that the density of $\mu_{t}$ is bounded away from 0 . This completes the estimate on bound pieces.

Estimate on free pieces. For free pieces, we adapt the estimates in [DHL, Section 6] and their modification due to the extra cutting by $\partial H$ in [BDM, Lemma 4.5]. We fix $n$ and estimate the mass of one-cylinders $\omega=X_{i}$ which are free when they enter $H$ for the first time at time $n$.

According to the construction in DHL, each one-cylinder $\omega$ is contained in a sequence of nested intervals $\omega \subset \omega^{(j)} \subset \omega^{(j-1)} \subset \cdots \subset \omega^{(1)}$ and corresponding times $s_{1}, \ldots, s_{j}$ such that $\left|f^{s_{i}}\left(\omega^{(i)}\right)\right| \geqslant$ $\delta_{1}$. Thus there is $\delta_{t}>0$ such that $m_{t}\left(f^{s_{i}}\left(\omega^{(i)}\right)\right) \geqslant \delta_{t}$ for each $i=1, \ldots, j$. We call the times $s_{i}$ growth times for $\omega$.

Define $E_{j}^{n}$ to be the set of one-cylinders $\omega$ such that $f^{n} \omega \subset H$ for the first time, $f^{n} \omega$ is free, and $\omega$ belongs to an interval which grows to fixed length $\delta_{1}$ precisely $j$ times before time $n$. Then

$$
\sum_{\substack{f^{n} \omega \subset H \\ \text { free }}} m_{t}(\omega) e^{\beta n}=\sum_{\substack{\omega \in E_{j}^{n} \\ j \leqslant \zeta n}} m_{t}(\omega) e^{\beta n}+\sum_{\substack{\omega \in E_{j}^{n} \\ \zeta n<j \leqslant n}} m_{t}(\omega) e^{\beta n}
$$

where $0<\zeta<1$ is determined below.

For $j \leqslant \zeta n$, we follow the proof of [DHL, Lemma 10] and [BDM, Lemma 4.5] to define $\left\{s_{1}=r_{1}\right\}$ as the set of points for which the first growth to length $\delta_{1}$ occurs at time $r_{1}$. We have by Proposition 3.2 ,

$$
m_{t}\left(s_{1}=r_{1}\right) \leqslant C_{0} e^{-\alpha r_{1}} \leqslant \frac{C_{0}}{\delta_{t}} e^{-\alpha r_{1}} m_{t}(X) .
$$

We then repeat this estimate on each element $f^{r_{1}} \omega$, which has $m_{t}$-measure at least $\delta_{t}$ by definition of $r_{1}$. Thus

$$
\left.m_{t}\left(x \in f^{r_{1}} \omega\right): s_{1} \geqslant r_{2}\right) \leqslant C_{0} e^{-\alpha r_{2}} \leqslant \frac{C_{0}}{\delta_{t}} e^{-\alpha r_{2}} m_{t}\left(f^{r_{1}} \omega\right) .
$$

By bounded distortion, this comprises a comparable fraction of the set in $\omega$, and thus

$$
m_{t}\left(s_{2}=r_{1}+r_{2}: s_{1}=r_{1}\right) \leqslant \frac{C_{0}^{2} D_{\delta}}{\delta_{t}^{2}} e^{-\alpha\left(r_{1}+r_{2}\right)},
$$

where $D_{\delta}$ is the distortion constant. Iterating this $j$ times, we have

$$
m_{t}\left(s_{j}=r_{1}+\cdots r_{j}: s_{1}=r_{1}, \ldots, s_{j-1}-s_{j-2}=r_{j-1}\right) \leqslant \frac{C_{0}^{j} D_{\delta}^{j-1}}{\delta_{t}^{j}} e^{-\alpha s_{j}} m_{t}(X) .
$$


Next we focus on the intervals $f^{s_{j}} \omega$ which lie inside $f^{s_{j}} \omega^{(j)}$ for a fixed $\omega^{(j)}$. In particular, we need to control the increase in complexity between times $s_{j}$ and $n$, i.e., the number of subintervals of $f^{s_{j}} \omega^{(j)}$ that will overlap when they enter $H$ at time $n-s_{j}$.

In $n-s_{j}$ iterates, $f^{s_{j}} \omega$ will enter $H$ for the first time. Along the way, due to the definition of $s_{j}$, $f^{i}\left(f^{s_{j}} \omega^{(j)}\right)$ cannot grow to length greater than $\delta_{1}$ or have a piece that makes a return to $X$, for $i=1, \ldots, n-s_{j}$. If $f^{i}\left(f^{s_{j}} \omega^{(j)}\right) \subset B_{\delta}(c)$ for some $c \in \mathrm{Crit}_{c}$, then a doubling may occur creating an overlap of subintervals in $f^{s_{j}} \omega^{(j)}$ when they enter $H$. We need to show that the expansion gained from time $s_{j}$ to time $n$ is sufficient to overcome this growth in complexity.

Let $p_{\delta}$ denote the minimum length of a bound period for $x \in B_{\delta}(c)$. According to [DHL, Lemma 2], for $x \in B_{\delta}(c)$, we have $\left|D f^{p+1}(x)\right| \geqslant \kappa^{-1} e^{\theta(p+1)}$ when $x$ reaches the end of its bound period of length $p$, where

$$
\theta=\frac{\Lambda-5 \vartheta_{c} \ell_{c}}{2 \ell_{c}}-\frac{\Lambda-5 \vartheta_{c} \ell_{c}}{2 \ell_{c} p_{\delta}}>0 .
$$

Note that the second term can be made arbitrarily small by choosing $\delta$ to be small (and therefore $p_{\delta}$ large). We choose $p_{\delta}$ sufficiently large that $e^{\theta p_{\delta}}>2^{1 / t}$. Define $\bar{\theta}=\theta-\frac{\log 2}{t p_{\delta}}$.

Now suppose that $\omega \subset \omega^{(j)}$ makes $k$ visits to $B_{\delta}(c)$ between times $s_{j}$ and $n$ and is free at time $n$. Then concatenating the expansion from [DHL, Lemma 2] and (C1), we have for $x \in f^{s_{j}} \omega$,

$$
\left|D f^{n-s_{j}}(x)\right| \geqslant \kappa \delta^{\ell_{\max }-1} e^{\bar{\gamma}\left(n-s_{j}\right)} 2^{\left(1+\frac{1}{p_{\delta}}\right) \frac{k}{t}},
$$

where $\bar{\gamma}=\min \{\gamma, \bar{\theta}\}$.

Since the complexity of $f^{i}\left(f^{s_{j}} \omega^{(j)}\right)$ increases at most by a factor of 2 with each entry into $B_{\delta}(c)$, we fix $\omega^{(j)}$ and let $A_{k}$ denote those $\omega \in E_{j}^{n}, \omega \subset \omega^{(j)}$, that make $k$ visits to $B_{\delta}(c)$ between times $s_{j}$ and $n$. Then

$$
\begin{aligned}
\sum_{\substack{\omega \subset \omega^{(j)} \\
\omega \in E_{j}^{n}}} m_{t}\left(f^{s_{j}} \omega\right) & \leqslant \sum_{k=0}^{n-s_{j}} \sum_{\omega \in A_{k}} C 2^{-\left(1+\frac{1}{p_{\delta}}\right) k} e^{-\bar{\gamma}\left(n-s_{j}\right) t} e^{p_{t}\left(n-s_{j}\right)} m_{t}\left(f^{n} \omega\right) \\
& \leqslant \sum_{k=0}^{n-s_{j}} C 2^{k} 2^{-\left(1+\frac{1}{p_{\delta}}\right) k} e^{-\bar{\gamma}\left(n-s_{j}\right) t} e^{p_{t}\left(n-s_{j}\right)} m_{t}(H) \\
& \leqslant C e^{\left(n-s_{j}\right)\left(-\bar{\gamma} t+p_{t}\right)} m_{t}(H),
\end{aligned}
$$

for some $C>0$.

Then since $m_{t}\left(f^{s_{j}} \omega^{(j)}\right) \geqslant \delta_{t}$, we iterate use (39) to iterate 38$)$ one more time to obtain,

$$
m_{t}\left(s_{1}=r_{1}, s_{2}-s_{1}=r_{2}, \ldots, n-s_{j}=r_{j+1}\right) \leqslant \frac{C_{0}^{j+1} D_{\delta}^{j}}{\delta_{t}^{j+1}} e^{-\theta_{1} n} m_{t}(H),
$$

where $\theta_{1}=\min \left\{\bar{\gamma} t-p_{t}, \alpha\right\}$. Summing over all possible $(j+1)$-tuples such that $\sum_{i} r_{i}=n$, we use the same combinatorial argument as in [DHL, Lemma 7] to bound their number by $e^{\eta n}$, where $\eta$ can be made as small as we like by choosing $\zeta$ sufficiently small (but holding $\delta$ fixed, which allows us to hold the distortion constant fixed). Thus,

$$
\sum_{\substack{\omega \in E_{j}^{n} \\ j \leqslant \zeta n}} m_{t}(\omega) e^{\beta n} \leqslant \frac{C_{1}}{\delta_{t}}\left(\frac{C_{1} D_{\delta}}{\delta_{t}}\right)^{\zeta n} e^{\left(-\theta_{1}+\beta+\eta\right) n} m_{t}(H),
$$

and choosing $\zeta$ and $\beta$ sufficiently small yields a bound exponentially small in $n$ times $m_{t}(H)$. 
Finally, we focus on those $\omega$ with $j>\zeta n$. Here we follow the proof of [DHL, Lemma 11] and its modification in [BDM, Lemma 4.5]. By [DHL, Lemma 1], every time a piece grows to length $\delta_{1}$, a fixed fraction, call it $\xi$, of $f^{s_{i}}\left(\omega^{(i)}\right)$ makes a full return to $X$ by a fixed time $s^{*}$. Since $m_{t}(X) \geqslant \delta_{t}$, we also know the portion that makes a full return by time $s^{*}$ constitutes a fixed fraction $\xi_{t}$ of the $m_{t}$ measure of $f^{s_{i}}\left(\omega^{(i)}\right)$. Due to bounded distortion, a fixed fraction $\xi_{t} / D_{\delta}$ of $\omega^{(i)}$ makes a return by time $s_{i}+s^{*}$. We now iterate this $j$ times, using the fact that each $\omega \in E_{j}^{n}$ belongs to an interval $\omega^{(j)}$ which also has its $j$ th growth time at time $s_{j}$. Thus

$$
\sum_{\omega \in E_{j}^{n}} m_{t}(\omega) \leqslant \sum_{\omega^{(j)} \in E_{j}} m_{t}\left(\omega^{(j)}\right) \leqslant\left(1-\frac{\xi_{t}}{D_{\delta}}\right)^{j} m_{t}(X),
$$

where $E_{j}$ is the set of $\omega^{(j)}$ corresponding to $E_{j}^{n}$. Note that once $\mathcal{H}(h)$ is fixed, neither $X$ nor $D_{\delta}$ changes as we shrink $h$. Moreover, the fraction $\xi_{t}$ that returns to $X$ by time $s^{*}$ does not deteriorate as $h$ decreases since a smaller hole does not prevent an interval from making its full return to $X$.

Now due to bounded distortion and letting $\left|D f^{s_{j}}\left(\omega^{(j)}\right)\right|$ denote the average value of $\left|D f^{s_{j}}\right|$ on $\omega^{(j)}$, we have $m_{t}\left(\omega^{(j)}\right) \geqslant D_{\delta}^{t} m_{t}\left(f^{s_{j}} \omega^{(j)}\right)\left|D f^{s_{j}}\left(\omega^{(j)}\right)\right|^{-t} e^{-s_{j} p_{t}}$. This, together with the previous estimate implies

$$
\sum_{\omega^{(j)} \in E_{j}}\left|D f^{s_{j}}\left(\omega^{(j)}\right)\right|^{-t} e^{-s_{j} p_{t}} \leqslant D_{\delta}^{-t}\left(1-\frac{\xi_{t}}{\mathcal{D}_{\delta}}\right)^{j} .
$$

Between time $s_{j}$ and time $n$, the complexity of $\omega$ entering $H$ can increase in the same way as described earlier. Thus we may combine (39) with (41) to obtain

$$
\begin{aligned}
\sum_{\omega \in E_{j}^{n}} m_{t}(\omega) & \leqslant \sum_{\omega(j) \in E_{j}} \sum_{\substack{\omega \subset \omega^{(j)} \\
\omega \in E_{j}^{n}}} \frac{m_{t}(\omega)}{m_{t}\left(f^{s_{j}} \omega\right)} m_{t}\left(f^{s_{j}} \omega\right) \\
& \leqslant \sum_{\omega^{(j)} \in E_{j}} D_{\delta}^{-t}\left|D f^{s_{j}}\left(\omega^{(j)}\right)\right|^{-t} e^{-s_{j} p_{t}} \sum_{\substack{\omega \subset \omega^{(j)} \\
\omega \in E_{j}^{n}}} m_{t}\left(f^{s_{j}} \omega\right) \\
& \leqslant C D_{\delta}^{-2 t}\left(1-\frac{\xi_{t}}{\mathcal{D}_{\delta}}\right)^{j} e^{\left(n-s_{j}\right)\left(-\bar{\gamma} t+p_{t}\right)} m_{t}(H) .
\end{aligned}
$$

Summing this estimate for $j>\zeta n$ yields

$$
\sum_{\substack{\omega \in E_{j}^{n} \\ \zeta n<j \leqslant n}} m_{t}(\omega) e^{\beta n} \leqslant C^{\prime}\left(1-\frac{\xi_{t}}{D_{\delta}}\right)^{\zeta n} e^{\beta n} m_{t}(H),
$$

and this can be made exponentially small in $n$ by choosing $\beta$ sufficiently small. Note that choosing $\beta$ small will force $H$ to be very small, but this is not a restriction since we are interested only in the small hole limit.

This estimate combined with 40 completes the estimate on the free pieces and the proof of Lemma 9.2 .

Proof of Lemma 9.3. Fix $0<\varsigma<s_{t}$ and $\eta>0$. By Lemma 9.5, there exists $\delta_{\varsigma}>0$ and a measurable set $E_{\delta_{\varsigma}}$ with $m_{t}\left(E_{\delta_{\varsigma}}\right)>1-\eta$ such that (36) holds for all $z \in E_{\delta_{\varsigma}}$ and all $\delta<\delta_{\varsigma}$.

Now choose $\delta$ so small that $2 \delta<\delta_{\varsigma}$. Then if $z \in E_{\delta_{\varsigma}} \cap B_{\delta e^{-\varsigma n}}\left(f^{n}(c)\right)$, we have by Lemma 9.5.

$$
m_{t}\left(B_{\delta e^{-\varsigma n}}\left(f^{n}(c)\right)\right) \leqslant m_{t}\left(B_{2 \delta e^{-\varsigma n}}(z)\right) \leqslant(4 \delta)^{s_{t}-\varsigma} e^{-n \varsigma\left(s_{t}-\varsigma\right)} .
$$


Let $J=\left\{n \in \mathbb{N}: B_{\delta e^{-\varsigma n}}\left(f^{n}(c)\right) \cap E_{\delta_{\varsigma}} \neq \emptyset\right\}$. Then it follows from the above estimate that

$$
m_{t}\left(\cup_{n \in J} B_{\delta e^{-\varsigma n}}\left(f^{n}(c)\right)\right) \leqslant \sum_{n \in J}(4 \delta)^{s_{t}-\varsigma} e^{-n \varsigma\left(s_{t}-\varsigma\right)} \leqslant(4 \delta)^{s_{t}-\varsigma} \frac{1}{1-e^{-\varsigma\left(s_{t}-\varsigma\right)}},
$$

and by shrinking $\delta$, we may make the quantity on the right hand side less than $\eta$. Now we estimate for all such $\delta$ sufficiently small,

$$
\begin{gathered}
m_{t}\left(z \in[0,1]: z \notin B_{\delta e^{-\varsigma n}}\left(f^{n}(c)\right) \forall n \in \mathbb{N}\right) \geqslant m_{t}\left(z \in E_{\delta_{\varsigma}}: z \notin B_{\delta e^{-\varsigma n}}\left(f^{n}(c)\right) \forall n \in \mathbb{N}\right) \\
\geqslant m_{t}\left(z \in E_{\delta_{\varsigma}}\right)-m_{t}\left(z \in E_{\delta_{\varepsilon}}: z \in B_{\delta e^{-\varsigma n}}\left(f^{n}(c)\right) \text { for some } n \in \mathbb{N}\right) \\
\geqslant m_{t}\left(z \in E_{\delta_{\varsigma}}\right)-m_{t}\left(\cup_{n \in J} B_{\delta e^{-\varsigma n}}\left(f^{n}(c)\right)\right) \geqslant 1-2 \eta .
\end{gathered}
$$

Since $\eta>0$ was arbitrary, this completes the proof of the lemma with respect to $m_{t}$. Since $\mu_{t} \ll m_{t}$, the property is generic with respect to $\mu_{t}$ as well.

9.3. Proof of periodic lemma. In this section we prove the necessary estimate to conclude Theorem 3.6 in the periodic case.

Proof of Lemma 9.4. The main idea of this proof is that by selecting $H$ appropriately, $\int_{\tilde{H}}\left(\mathcal{L}_{\phi_{t}}^{n}-\right.$ $\left.\mathcal{L}_{\phi_{t}^{H}}^{n}\right) g_{t}^{0} d \bar{m}_{t}$ is comparable to the measure of the set $\hat{H}:=H \cap f^{-p}(H)$. Once we have shown this, we use the fact that the density at $z$ exists and lies in $(0, \infty)$ to deduce that

$$
\frac{\mu_{t}(\hat{H})}{\mu_{t}(H)} \sim \frac{m_{t}(\hat{H})}{m_{t}(H)} \sim e^{S_{p} \phi(z)},
$$

where the final estimate is immediate by conformality, and $\sim$ denotes a uniform constant depending only on $f$ and $t$ (not $H$ ).

Recall that by construction, if a domain $X_{\varepsilon}^{i}$ has an iterate $k$ such that $f^{k}\left(X_{\varepsilon}^{i}\right) \cap H \neq \emptyset$ then $f^{k}\left(X_{\varepsilon}^{i}\right) \subset H$. Hence if we fix a column $i$, then every time that an iterate $f_{\Delta}^{k}\left(\Delta_{i, 0}\right)$ projects to intersect $H$, then in fact $\pi\left(f_{\Delta}^{k}\left(\Delta_{i, 0}\right)\right) \subset H$. We set $\mu_{\Delta, t}=g_{t}^{0} \bar{m}_{t}$.

Note that $\mathcal{L}_{\phi_{t}^{H}}^{n}$ only includes preimages of points in $\tilde{H}$ which enter $\tilde{H}$ for the first time at time $n$, while $\mathcal{L}_{\phi_{t}}^{n}$ counts all preimages of points in $\tilde{H}$ which enter $H$ at time $n$. Thus to estimate the quantity $\int_{\tilde{H}}\left(\mathcal{L}_{\phi_{t}}^{n}-\mathcal{L}_{\phi_{t}^{H}}^{n}\right) g_{t}^{0} d \bar{m}_{t}$, we sum the $\mu_{\Delta, t}$ measure on the tower of the set of points which both project to the hole $H$ at time $n$, as well as doing so at some previous time $0 \leqslant k \leqslant n-1$.

Fixing $n$, we choose $h$ so small that for $H \in \mathcal{H}(h)$, if $x \in H$, but $f^{p}(x) \notin H$ then $f^{k}(x) \notin H$ for $k=1, \ldots, n$. In particular, this means that if a point in a column of $\Delta$ projects to $H$ then the only way an $f_{\Delta}$-iterate of $x$ can project to $H$ again before time $n$ is if $\pi x$ was actually in some subset $f^{-i p}(H) \cap H$ for $i \geqslant 1$. Moreover, the $f_{\Delta}$-orbit of $x$ cannot return to the base $\Delta_{0}$ and then later project to the hole again before time $n$ since $H$ is so small that it cannot grow to length $\delta_{1}$ by time $n$.

Fix a column $i$ and suppose that at some level the projection $f_{\Delta}^{k}\left(\Delta_{i, 0}\right)$ is inside $H$. If this only happens once, then we don't count it. If it happens exactly twice then we count the set of points which when iterated forwards $n$ times project to the hole for the second time. Notice that since $\mu_{\Delta, t}$ is $f_{\Delta}$-invariant, the set of points which when iterated forwards $n$ times projected to the hole for the first time has the same measure as $\Delta_{i, 0}$, i.e., $\mu_{\Delta, t}\left(\Delta_{i, 0}\right)$. Continuing in the same way, we see that if parts of the column project to the hole exactly $k$ times, then we measure $(k-1) \mu_{\Delta, t}\left(\Delta_{i, 0}\right)$. Note that we only continue up to time $n$, so since within a column we can only repeatedly hit the 
hole every $p$ iterates, this process stops when $k>\frac{n}{p}$. Therefore,

$$
\int_{\tilde{H}}\left(\mathcal{L}_{\phi_{t}}^{n}-\mathcal{L}_{\phi_{t}^{H}}^{n}\right) g_{t}^{0} d \bar{m}_{t}=\sum_{k=2}^{\left\lfloor\frac{n}{p}\right\rfloor}(k-1) \sum_{\left\{i: \pi \Delta_{i, j_{q}} \subset H \text { for } k \text { times } j_{q}\right\}} \mu_{\Delta, t}\left(\Delta_{i, 0}\right) .
$$

Now notice that our setup implies that, as for $H$ itself, if $\pi \Delta_{i, j} \cap \hat{H} \neq \emptyset$ then $\pi \Delta_{i, j} \subset \hat{H}$, so the measure of $\hat{H}$ is by definition

$$
\sum_{\left\{i, j: \pi \Delta_{i, j} \subset \hat{H}\right\}} \mu_{\Delta, t}\left(\Delta_{i, j}\right) .
$$

If we consider this sum column by column, we can separate it into terms where the column projects to $\hat{H} k$ times, and noting that each element $\Delta_{i, j}$ has the same $\mu_{\Delta, t}$-measure, we obtain (suggestively using index $k-1$ rather than $k$ )

$$
\mu_{t}(\hat{H})=\sum_{k \geqslant 2}(k-1) \sum_{\left\{i: \pi \Delta_{i, j_{q}} \subset \hat{H} \text { for } k-1 \text { times } j_{q}\right\}} \mu_{\Delta, t}\left(\Delta_{i, 0}\right) .
$$

But since once a domain has $\pi \Delta_{i, j_{q}} \subset \hat{H}$ and $\pi f_{\Delta}^{p}\left(\Delta_{i, j_{q}}\right) \cap \hat{H}=\emptyset$, we must also have $\pi f_{\Delta}^{p}\left(\Delta_{i, j_{q}}\right) \subset$ $H \backslash \hat{H}$, and moreover, $f_{\Delta}^{p}\left(\Delta_{i, j_{q}}\right)$ is still in column $i$. Therefore, the values in (42) and (43) are the same, up to the measure of $H \cap f^{-p\left\lfloor\frac{n}{p}\right\rfloor}(H)$ which we claim is of order $e^{S_{n} \phi(z)}$ and is exponentially small in $n$.

To see this, note that by (H2) and choice of $\delta_{0}$, the orbit of $z$ is always 'free.' Thus by $(\mathrm{C} 1)$, we have $\left|D f^{i}(z)\right| \geqslant \kappa \delta_{0}^{\ell_{\max }-1} e^{\gamma i}$ for any $i \in \mathbb{N}$. A similar bound holds at time $n$ for any $x \in H \cap f^{-p\left\lfloor\frac{n}{p}\right\rfloor}(H)$ using bounded distortion, and the claim follows.

9.4. Specific classes of maps satisfying our assumptions: the proof of Theorem 3.7. We next assert that there is a reasonable class of maps with periodic points satisfying (31). Note that we expect the conclusions of the following lemma to hold for a much larger class of maps and periodic points.

Lemma 9.6. Let $z_{4}$ be a repelling periodic point of $f_{4}$ not lying on the critical orbit. Consider its hyperbolic continuation $z_{\lambda}$ for $\lambda$ close to 4 (i.e. $z_{\lambda}$ has a topologically identical orbit under $f_{\lambda}$ as $z_{4}$ does under $\left.f_{4}\right)$. Then for any $\varsigma>0$ there exist $t_{0}<1<t_{1}$ and a positive Lebesgue measure set of parameters $\Omega^{\prime}=\Omega^{\prime}\left(z_{4}\right)$ such that whenever $\lambda \in \Omega^{\prime}$ and $t \in\left[t_{0}, t_{1}\right]$, then $f_{\lambda}$ has an equilibrium state $\mu_{t}$ and there exists $\delta_{z_{\lambda}}>0$ such that

$$
\left|f^{n}(c)-z_{\lambda}\right| \geqslant \delta_{z_{\lambda}} e^{-n \varsigma} \text { for all } n \geqslant 0 .
$$

In particular, (31) holds for $z_{\lambda}$.

Proof. The proof is the same as for [FFT, Theorem 7]. There it is shown that there is an acip for $f_{\lambda}$, but since this has exponential tails, BT] implies that $\mu_{t}$ also exists. Moreover, they show that (44) holds.

Proof of Theorem 3.7. We focus on the periodic case, since the tools required for the generic case are almost classical. This means that we wish to prove condition $(\mathrm{P})$ for our family of maps $f_{\lambda}$ and periodic points $z_{\lambda}$, as well as noting that $\hat{\delta}$ in Lemma 9.5 can be taken uniformly. We consider the family of maps $\Omega^{\prime}\left(z_{4}\right)$ given by Lemma 9.6. We notice that if we fix the constants $\nu_{c}, \Lambda, \alpha, \beta, t$ then, possibly by restricting our class of maps, we also get a uniform estimate on $s_{t}$ and so by Lemma 9.6 . Lemma 9.2 holds throughout our family. We also use the fact here that $\mu_{t}$ and $m_{t}$ do not change 
too much within this family due to statistical stability (see [FT]), so the constants coming from the measure $m_{t}$ of small intervals can also be taken to depend only on the family. Hence (31) holds.

To complete the proof of the lemma we must show that the density $\frac{d \mu_{\lambda, t}}{d m_{\lambda, t}}$ is bounded at $z_{\lambda}$. This follows almost exactly as in [N], in particular Corollary 4.2. The problem was expressed there as finding a uniform bound on $\left(\mathcal{L}_{\phi_{1}}^{n} 1\right)(z)$. The main issue was to estimate the distortion of $f^{n}$ along orbits which are relevant to this transfer operator, which was guaranteed when

$$
\sum_{n=0}^{\infty} \frac{1}{\left|D f^{n}(f(c))\right|^{\ell_{c}}\left|f^{n}(f(c))-z\right|^{1-\frac{1}{\ell_{c}}}}<\infty .
$$

Clearly this holds in our case by (31) and the exponential growth of derivative along the critical orbit. For our case, for each $t$ in a neighborhood of 1 we are interested in finding a uniform bound on $\left(\mathcal{L}_{\phi_{t}}^{n} 1\right)(z)$, that is showing

$$
\sum_{n=0}^{\infty} \frac{1}{\left(\left|D f^{n}(f(c))\right|^{\ell_{c}}\left|f^{n}(f(c))-z\right|^{1-\frac{1}{\ell_{c}}}\right)^{t} e^{n p(t)}}<\infty .
$$

Clearly for $t$ close to 1 , the fact that this is bounded holds analogously to the case when $t=1$, i.e., (44) above.

Remark 9.7. Note that the above proof of the boundedness of the density was closely tied to (31). The proof in $[\mathrm{N}]$ requires a negative Schwarzian condition along with unimodality. We would expect this to extend beyond that setting.

\section{REFERENCES}

[BDM] H. Bruin, M. Demers, I. Melbourne, Existence and convergence properties of physical measures for certain dynamical systems with holes, Ergodic Theory Dynam. Systems 30 (2010) 687-728.

[BK] H. Bruin and G. Keller, Equilibrium states for S-unimodal maps, Ergodic Theory Dynam. Systems 18 (1998) 765-789.

[BS] H. Bruin, S. van Strien, Expansion of derivatives in one-dimensional dynamics, Israel. J. Math. 137 (2003) $223-263$.

[BT] H. Bruin, M. Todd, Equilibrium states for interval maps: the potential $-t \log |D f|$, Ann. Sci. École Norm. Sup. (4) 42 (2009) 559-600.

[B] J. Buzzi, Markov extensions for multidimensional dynamical systems, Israel J. Math. 112 (1999) 357-380.

[CT] Y.M. Chung, H. Takahasi, Multifractal formalism for Benedicks-Carleson quadratic maps, Ergodic Theory Dynam. Systems 34 (2014) 1116-1141.

[DW] M.F. Demers, P. Wright, Behaviour of the escape rate function in hyperbolic dynamical systems, Nonlinearity 25 (2012) 2133-2150.

[DWY] M.F. Demers, P. Wright, L.-S. Young, Entropy, Lyapunov exponents and escape rates in open systems, Ergodic Theory Dynam. Systems 32 (2012) 1270-1301.

[DY] M.F. Demers, L.-S. Young, Escape rates and conditionally invariant measures, Nonlinearity 19 (2006) $377-397$.

[DHL] K. Díaz-Ordaz, M. Holland and S. Luzzatto, Statistical properties of one-dimensional maps with critical points and singularities, Stoch. Dyn. 6 (2006) 423-458.

[Do] N. Dobbs On cusps and flat tops, Ann. Inst. Fourier (Grenoble) 64 (2014) 571-605.

[FP] A. Ferguson, M. Pollicott, Escape rates for Gibbs measures, Ergodic Theory Dynam. Systems 32 (2012) 961988.

[FFT] A.C. Freitas, J.M. Freitas, M. Todd, The compound Poisson limit ruling periodic extreme behaviour of nonuniformly hyperbolic dynamics, Comm. Math. Phys. 321 (2013) 483-527.

[FT] J.M. Freitas, M. Todd, The statistical stability of equilibrium states for interval maps, Nonlinearity 22 (2009) 259-281.

[Ho] F. Hofbauer, Local dimension for piecewise monotonic maps on the interval, Ergodic Theory Dynam. Systems 15 (1995) 1119-1142.

[I] G. Iommi, Multifractal analysis for countable Markov shifts, Ergodic Theory Dynam. Systems 25 (2005), 1881-1907. 
[IT1] G. Iommi, M. Todd, Natural equilibrium states for multimodal maps, Comm. Math. Phys. 300 (2010) 65-94.

[IT2] G. Iommi, M. Todd, Dimension theory for multimodal maps, Ann. Henri Poincaré 12 (2011) 591-620.

[IT3] G. Iommi, M. Todd, Thermodynamic formalism for interval maps: inducing schemes, Dyn. Syst. 28 (2013) 354-380.

[KL1] G. Keller, C. Liverani, Stability of the spectrum for transfer operators, Annali della Scuola Normale Superiore di Pisa, Scienze Fisiche e Matematiche, (4) XXVIII (1999), 141-152.

[KL2] G. Keller, C. Liverani, Rare events, escape rates and quasistationarity: some exact formulae, J. Stat. Phys. 135 (2009) 519-534.

[L] F. Ledrappier, Some properties of absolutely continuous invariant measures on an interval, Ergodic Theory Dynam. Systems 1 (1981) 77-93.

[LM] C. Liverani, V. Maume-Deschamps, Lasota-Yorke maps with holes: conditionally invariant probability measures and invariant probability measures on the survivor set, Ann. Inst. H. Poincaré Probab. Statist. 39 (2003) 385412.

[M] V. Maume-Deschamps, Projective metrics and mixing properties on towers, Trans. Amer. Math. Soc. 353 (2001) 3371-3389.

[N] T. Nowicki, Some dynamical properties of S-unimodal maps, Fund. Math. 142 (1993) 45-57.

[PS] Y. Pesin and S. Senti, Equilibrium measures for maps with inducing schemes J. Mod. Dyn. 2 (2008) 1-31.

[Pr] F. Przytycki, Lyapunov characteristic exponents are nonnegative, Proc. Amer. Math. Soc. 119 (1993) $309-317$.

[PU] F. Przytycki, M. Urbański, Conformal fractals: ergodic theory methods, London Mathematical Society Lecture Note Series. CUP, 2010.

[Ra] P. Raith, Hausdorff dimension for piecewise monotonic maps, Studia Math. 94 (1989) 17-33.

[Ro] V.A. Rohlin, Exact endomorphisms of a Lebesgue space, Izv. Akad. Nauk SSSR Ser. Mat. 25 (1961) $499-530$.

[Se] S. Senti, Dimension of weakly expanding points for quadratic maps, Bull. Soc. Math. France 131 (2003) $399-$ 420.

[Y] L.-S. Young, Statistical properties of dynamical systems with some hyperbolicity, Ann. of Math. 147 (1998) 585-650.

[Z] R. Zweimüller, Invariant measures for general(ized) induced transformations, Proc. Amer. Math. Soc. 133 (2005) 2283-2295.

Mark F. Demers, Department of Mathematics, Fairfield University, Fairfield, CT 06824, USA

E-mail address: mdemers@fairfield.edu

$U R L:$ http://www.faculty.fairfield.edu/mdemers/

Mike Todd, Mathematical Institute, University of St Andrews, North Haugh, St Andrews, KY16 9SS, SCOTLAND

E-mail address: m.todd@st-andrews.ac.uk

$U R L:$ http://www.mcs.st-and.ac.uk/ miket/ 